Supplementary Material 


\title{
Molecular Crystal Global Phase Diagrams: II. Reference Lattices
}

\author{
by R. B. McClurg and J. B. Keith
}

Notes to Accompany

Table 6: Structure Classification 


\section{Sphere Packing}

See MEZDIE01 on pages 14 and 15. Notes refer to the hand-written letters on the following pages.

A. CSD entry MEZDIE01 is described using space group 2 with a single molecule in the asymmetric unit with center of mass located at the Wyckoff site i. The HermannMauguin label ( $\left.\mathrm{P}_{-}-1\right)$ is provided for convenience.

B. Unit cell parameters from MEZDIE01.cif

C. Non-dimensional unit cell parameters using the a-axis length as the reference length

D. Notes based on the neighbor histogram (separate document) indicating that there are 6 nearly equi-distant neighbors that are not in a single hemisphere. Therefore the structure appears to approximate a sphere packing. The remainder of the entry serves to classify the packing.

E. Matrix representation for the parameters in $\mathrm{C}$ above. Euclidean norms of the columns give the desired magnitudes $(1,1.4527186$, and 0.9209443$)$. Dot products of the columns yield the product of the column norms and the cosine of the included angle according to the cosine law. The angles have the expected values $(90.47694,111.67244$, and 89.98776 degrees). See Appendix A, equation 1.

F. There is a single molecule in the asymmetric unit at Wyckoff site " $\mathrm{i}$ " with point symmetry "1".

G. The fractional center-of-mass coordinates are provided for both molecules in the unit cell. See Appendix A, equations 2 and 3.

H. A transformation matrix is provided which halves the volume of the unit cell (Determinant $=1 / 2$ ), rotates the unit cell, and shifts the origin. Halving the volume of the cell recognizes that the center-of-mass lattice has a single molecule in the primitive unit cell. [The observed unit cell has two molecules in the unit cell due to orientation of the non-spherical molecules and minor translations of their centers.] See Appendix A, equations $4,5,7$, and 8 .

I. The inverse transformation matrix is calculated for use below.

$\mathrm{J}$. The dot product of the matrix representation (E above) and the upper-left transformation submatrix (H above) yields the transformed matrix representation. See Appendix A, equation 6. Unit cell parameters are calculated using Euclidean norms and the cosine law as described in $\mathrm{E}$ above.

K. The Niggli matrix, normalized using the (new) a-axis length is provided.

L. The Niggli matrix conforms to the "Main Conditions" for a Type II reduced unit cell. Comparing the Niggli matrix to tabulated character definitions reveals that the reduced cell is triclinic (character 44), but close to body-centered-cubic (cI, character 5).

M. Transforming the fractional center-of-mass coordinates $(\mathrm{G})$ using the inverse transformation matrix (I) as indicated in the Appendix, equations 9 and 10, yields the indicated coordinates. Both molecules are near the origin $(0,0,0)$ of a unit cell (modulo unity). This is a necessary condition for successful unit cell volume reduction $(\mathrm{H})$. There is one molecule in the reduced unit cell $\left(Z^{\prime}=1\right)$. See Appendix A, equations 9 and 10.

$\mathrm{N}$. The observed unit cell (MEZDIE01), called the daughter, was observed in a space group with two symmetry operations $(|G|=2)$ and two molecules per primitive unit cell $(Z=2)$ yielding a symmetry density of one $(|\mathrm{G}| / \mathrm{Z}=1)$. The identified $\mathrm{BCC}$ reference lattice 
(space group 229) has 48 symmetry operations per primitive unit cell $(|G|=48)$ and one molecule per primitive unit cell $\left(Z^{\prime}=1\right.$ as indicated in $\mathrm{M}$ above). The ratio yields a symmetry density of 48 for the $\mathrm{BCC}$ reference lattice. The ratios of the $\mathrm{Z}$ values gives the "size" and ratios of the symmetry densities gives the "index" of the symmetrybreaking transformation in passing from the reference lattice to the daughter cell. Note that the ratios are in opposite senses.

O. The transformation from the reduced basis for character 5 to the conventional basis is tabulated along with the character definitions. See Appendix A, equations 11, 12, and 14.

$\mathrm{P}$. The matrix inverse of the product of the transformation matrices $(\mathrm{H}$ and $\mathrm{O})$ yields the overall transformation matrix from the reference lattice (BCC) to the observed structure. The determinant of the transformation is unity since the conventional BCC reference cell and MEZDIE01 each contain two molecules. See Appendix A, equations 15 and 16.

Q. COPL indicates that the overall transformation matrix is consistent with the indicated symmetry-breaking transition with size 2 and index 48 as calculated in $\mathrm{N}$ above. The order parameter is coupled. 


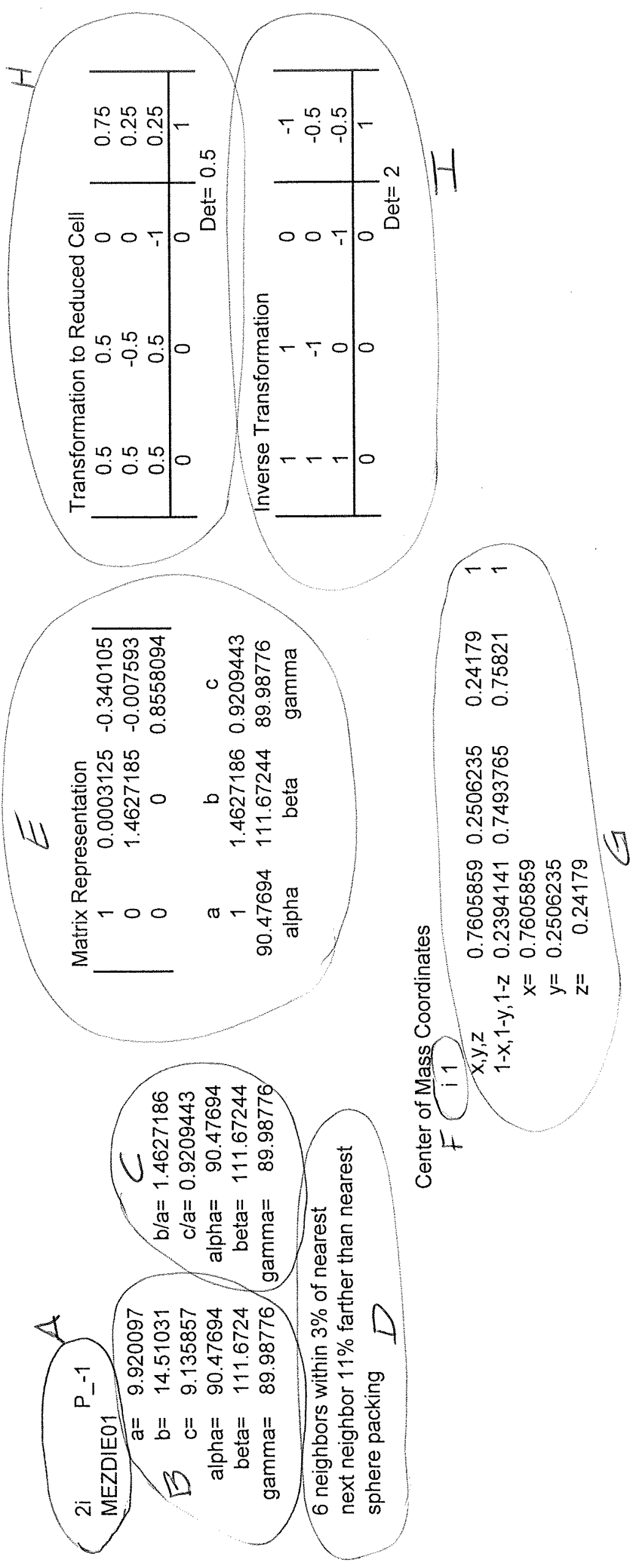




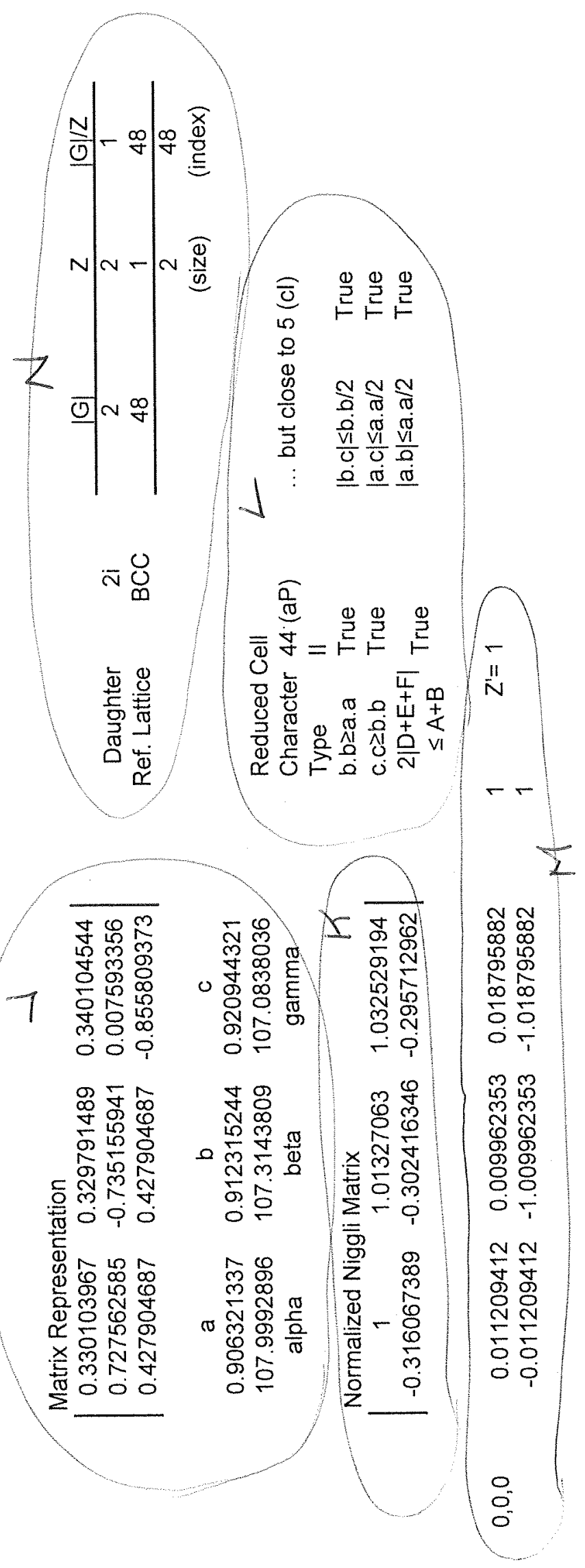




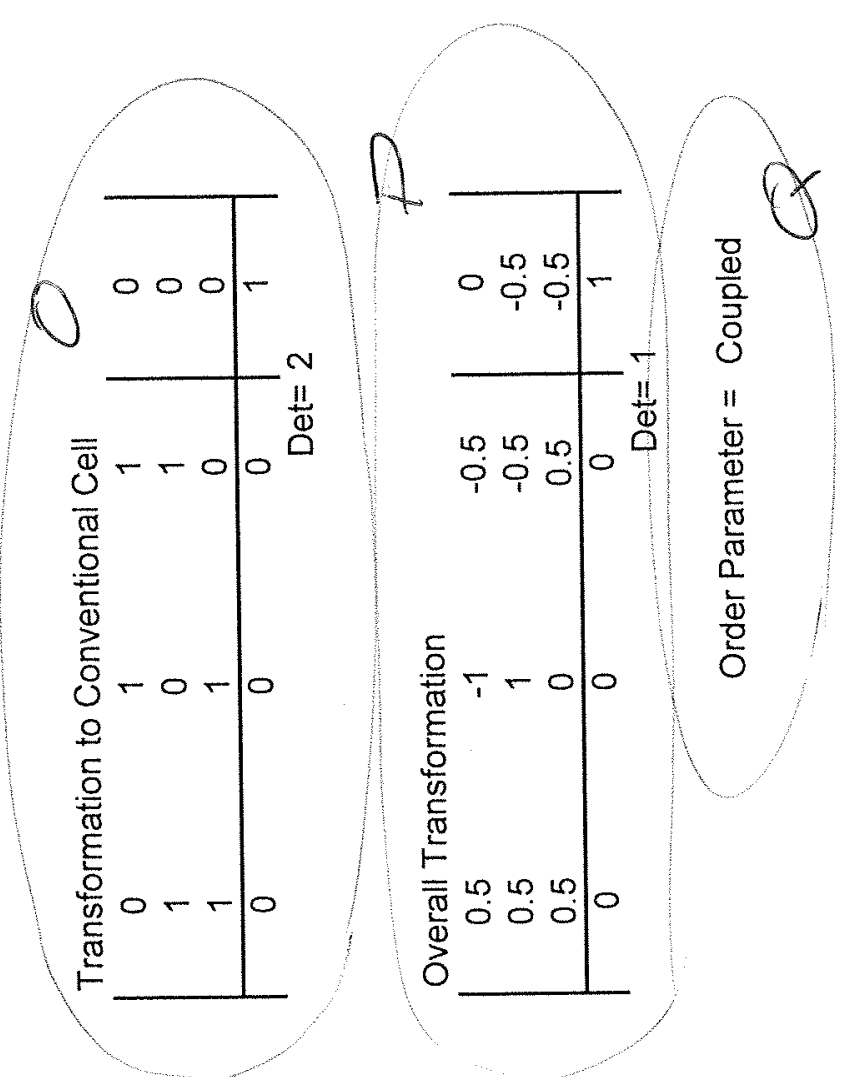




\section{Rod Packing}

See MECKIO on page 10. Notes refer to the hand-written letters on the following pages.

A-D. See corresponding notes for MEZDIE01. For this structure, the histogram indicates that each molecule has two nearest neighbors forming rods. The rods are shown to have $\mathrm{p}-42 \mathrm{~m}$ rod symmetry and pack in a nearly hexagonal arrangement.

E. The indicated matrix transformation is analogous to that for MEZDIE01 above. See Appendix B, equation 19.

F. The molecular centers-of-mass reside at Wyckoff point e with point symmetry $\mathrm{m}$. The fractional coordinates are provided. See Appendix B, equations 20 and 21.

G. Four sites on the 3-fold rotation axes of the molecule are indicated. They are used to determine the rod symmetry below.

$H$. The indicated transformation matrix puts the rod axis along the $x$-axis, makes the $y$ - and $\mathrm{z}$-axes perpendicular to the rod axis, and shifts the origin to the center-of-mass of a selected molecule. See Appendix B, equations 22 and 23.

I. The inverse of the transformation matrix is provided for use in transforming fractional coordinates.

J. The rod axis, rod origin, and adjustable parameter in $\mathrm{H}$ are extracted from $\mathrm{H}$ for convenience.

K. A second transformation rotates the rod about its axis to place the rod in standard orientation and rescales the coordinates perpendicular to the rod axis to make them equal in magnitude. See Appendix B, equations 24 and 25.

L. The unit cell matrix representation (E) transformed by $\mathrm{H}$ and $\mathrm{K}$ yield the matrix representation $\mathrm{L}$. The unit cell is tetragonal by design.

M. The center-of-mass of the reference molecule is at the origin by design. The three-fold axes of the molecule very closely follow the Wyckoff orbit $f$ of a p $-42 \mathrm{~m}$ rod symmetry in the $2^{\text {nd }}$ setting.

$\mathrm{N}$. There is one molecule in the rod unit cell.

$\mathrm{O}$. The symmetry-breaking transformation from rods to the observed structure has size 2 and index 4. See the analogous note $\mathrm{N}$ for MEZDIE01 above. 


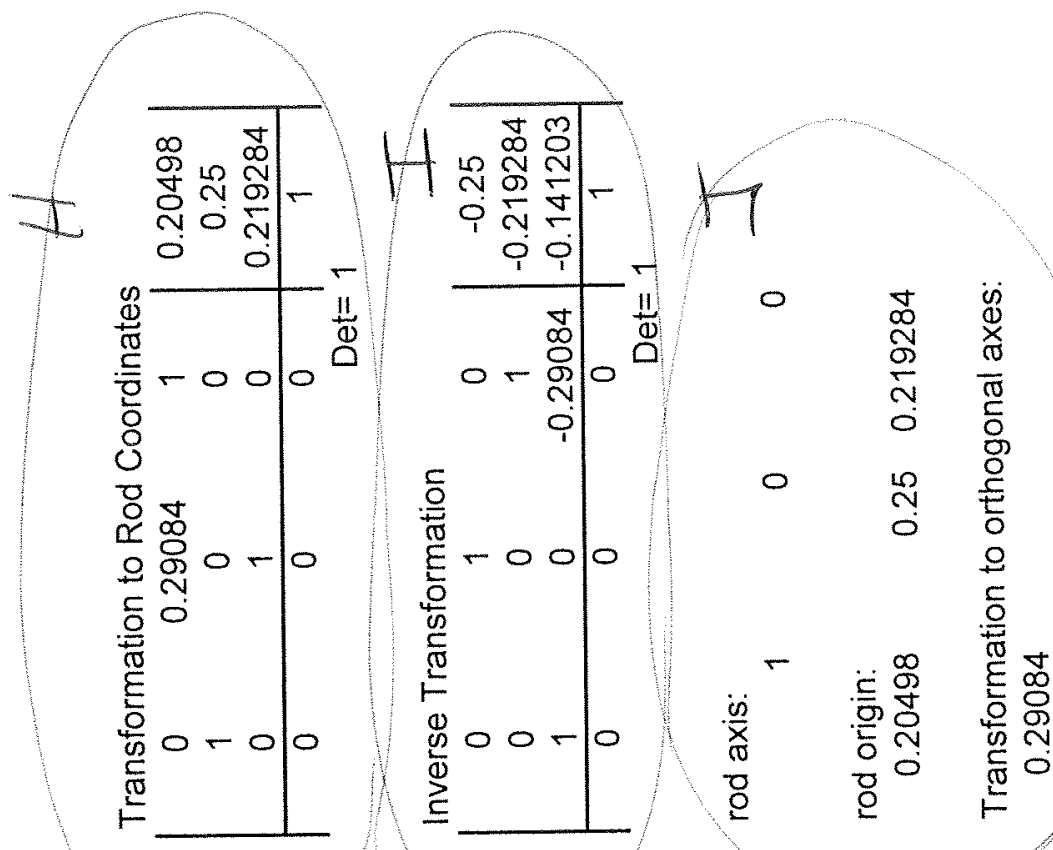

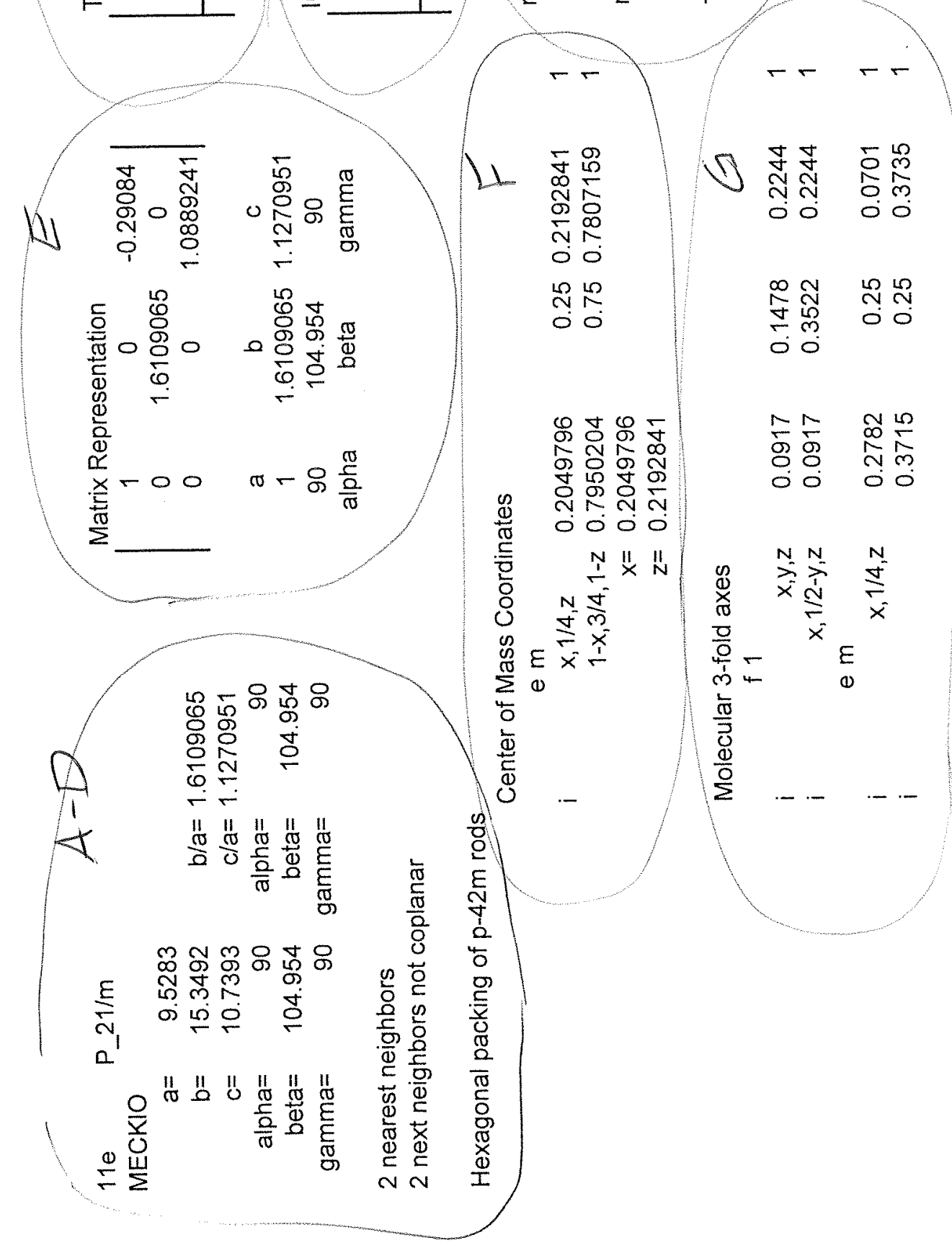




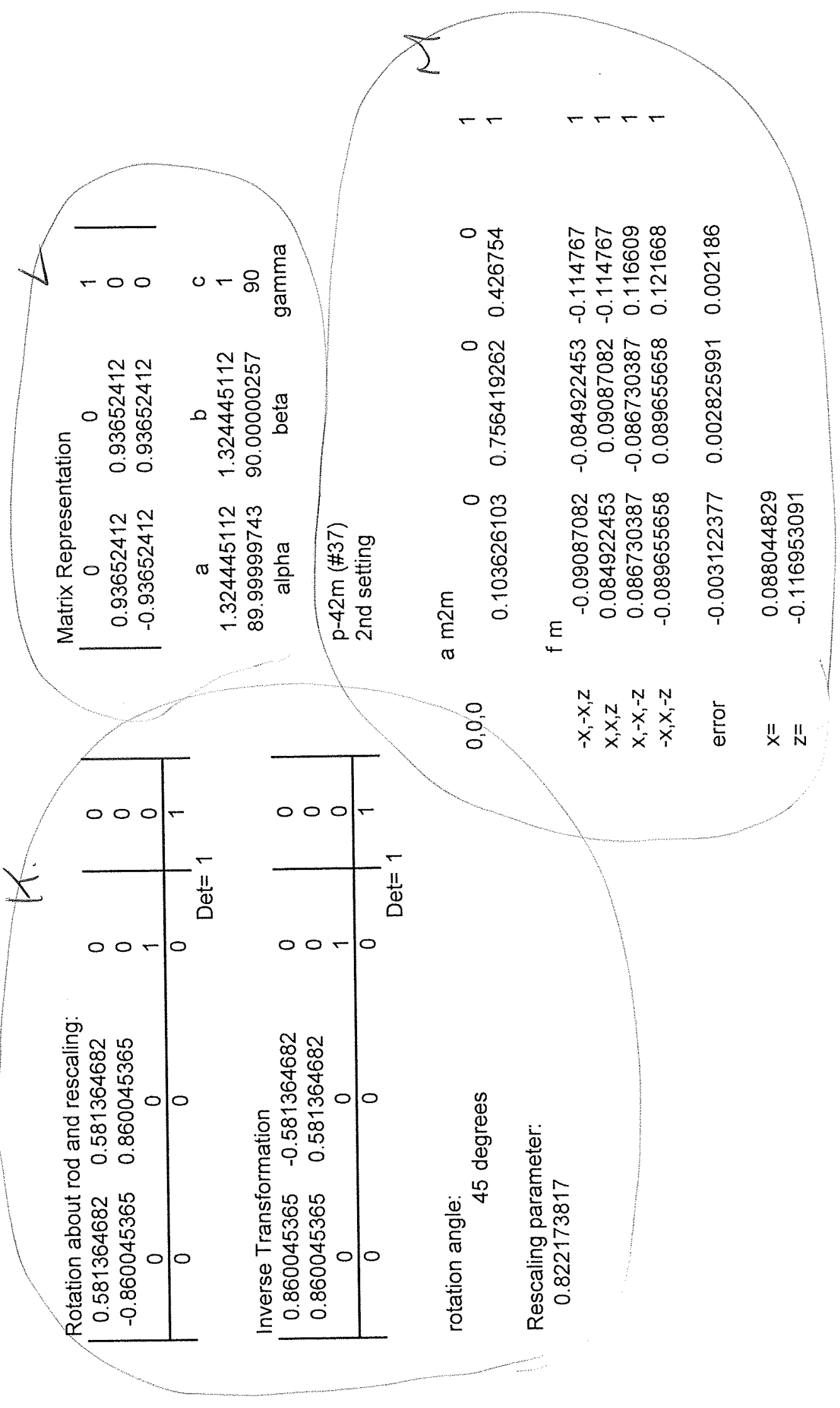



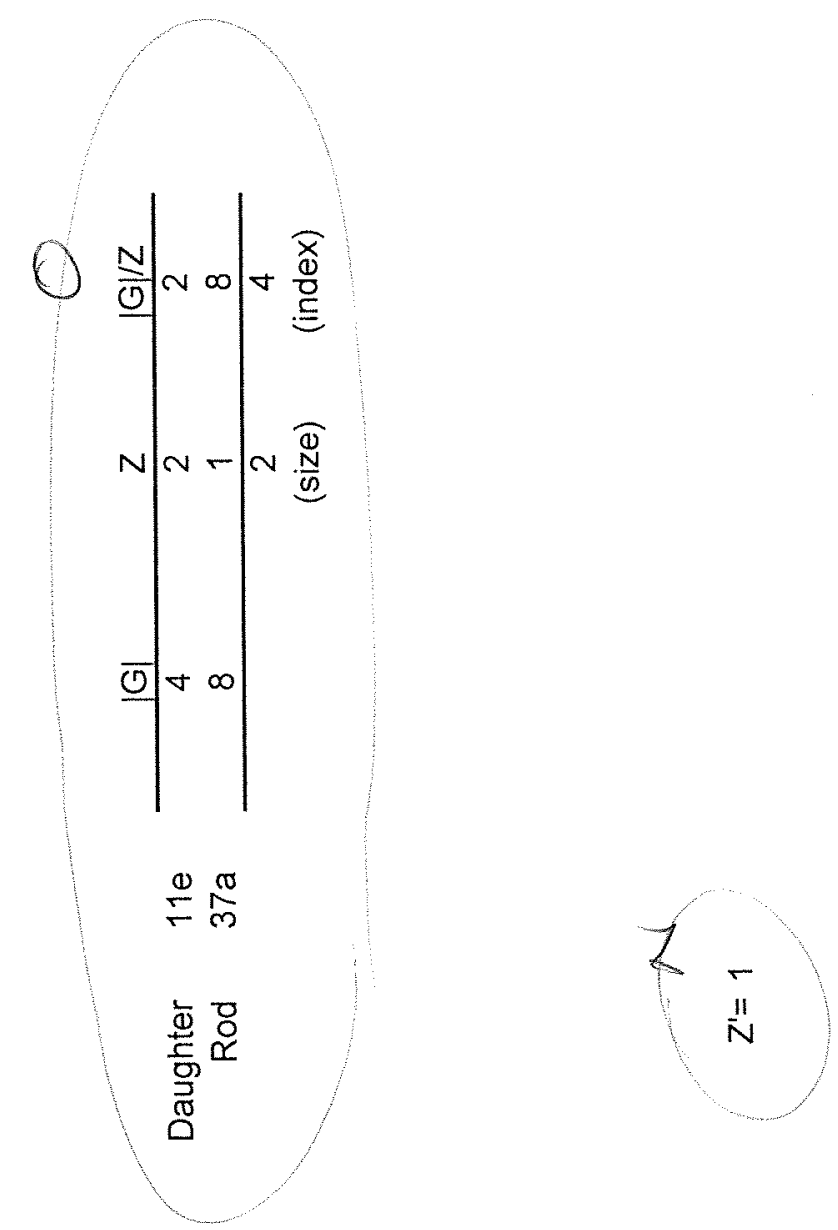


\section{Planar Packing}

See CAMPOV on page 10. Notes refer to the hand-written letters on the following pages.

A-E. See corresponding notes for MEZDIE01. For this structure, the histogram indicates that each molecule has three nearest neighbors forming two-dimensional planes. The planes are shown to have $\mathrm{p} 2 \mathrm{gg}$ projection symmetry.

F. The molecular centers-of-mass reside at Wyckoff point e with point symmetry 1 . Fractional coordinates are provided.

G. The indicated transformation matrix puts the plane into orthogonal axes. The inverse transformation is used to compute fractional center-of-mass coordinates in the transformed frame.

H. No rotation nor any rescaling of axes are needed to put the plane into standard orientation.

I. The matrix representation of the unit cell $(\mathrm{E})$, after the indicated transformations ( $\mathrm{G}$ and $\mathrm{H})$, is an orthorhombic cell.

J. Transforming the fractional center-of-mass coordinates $(F)$ using the inverse transformation matrix $(\mathrm{G})$ yields the coordinates indicated. There are four molecules in the primitive cell.

$\mathrm{K}$. The fractional coordinates $(\mathrm{J})$ are consistent with $\mathrm{p} 2 \mathrm{gg}$ projection symmetry (neglecting the z-coordinate) and $2 / 1 \mathrm{c}$ symmetry when considering the third dimension.

L. The symmetry-breaking transformation from planes to the observed structure has size 1 and index 1 . See the analogous note $\mathrm{N}$ for MEZDIE above. 


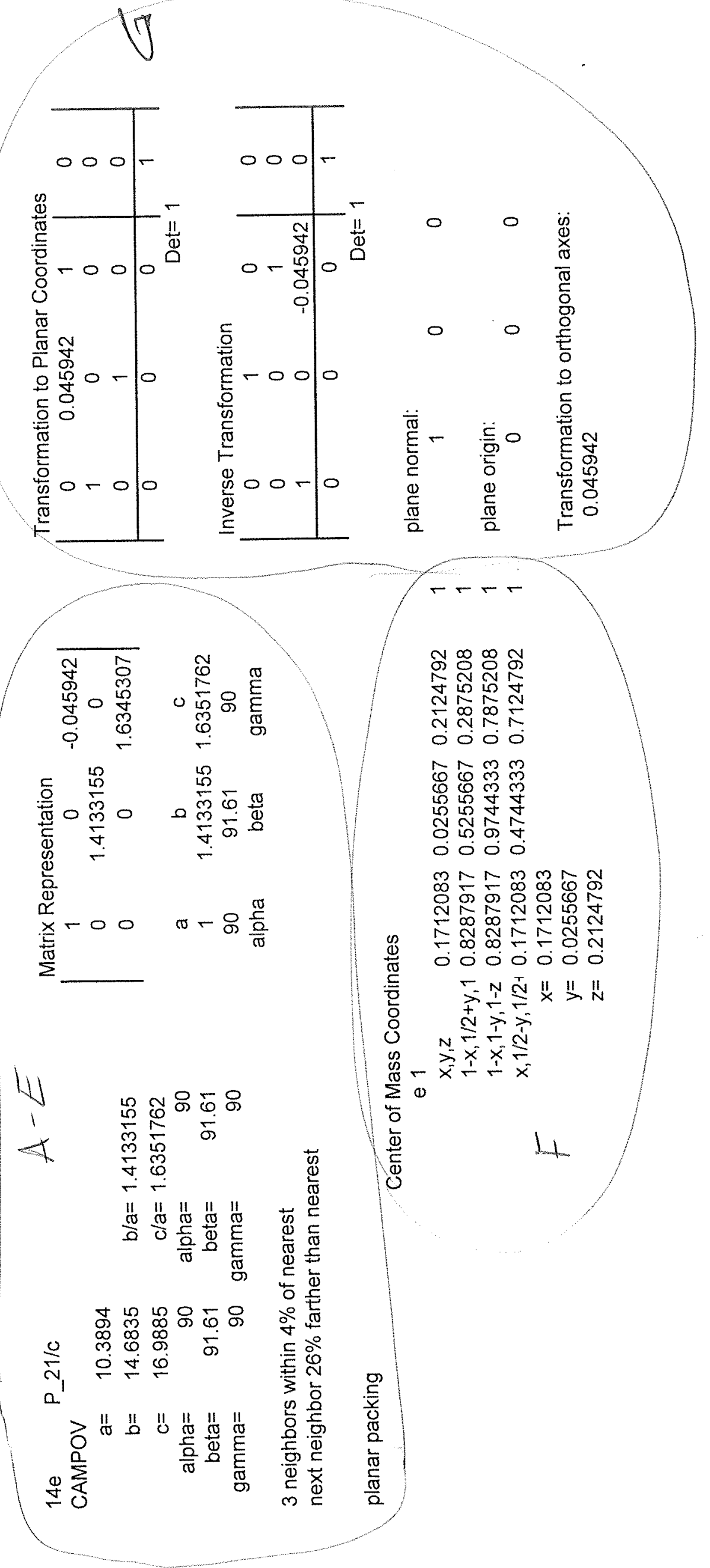




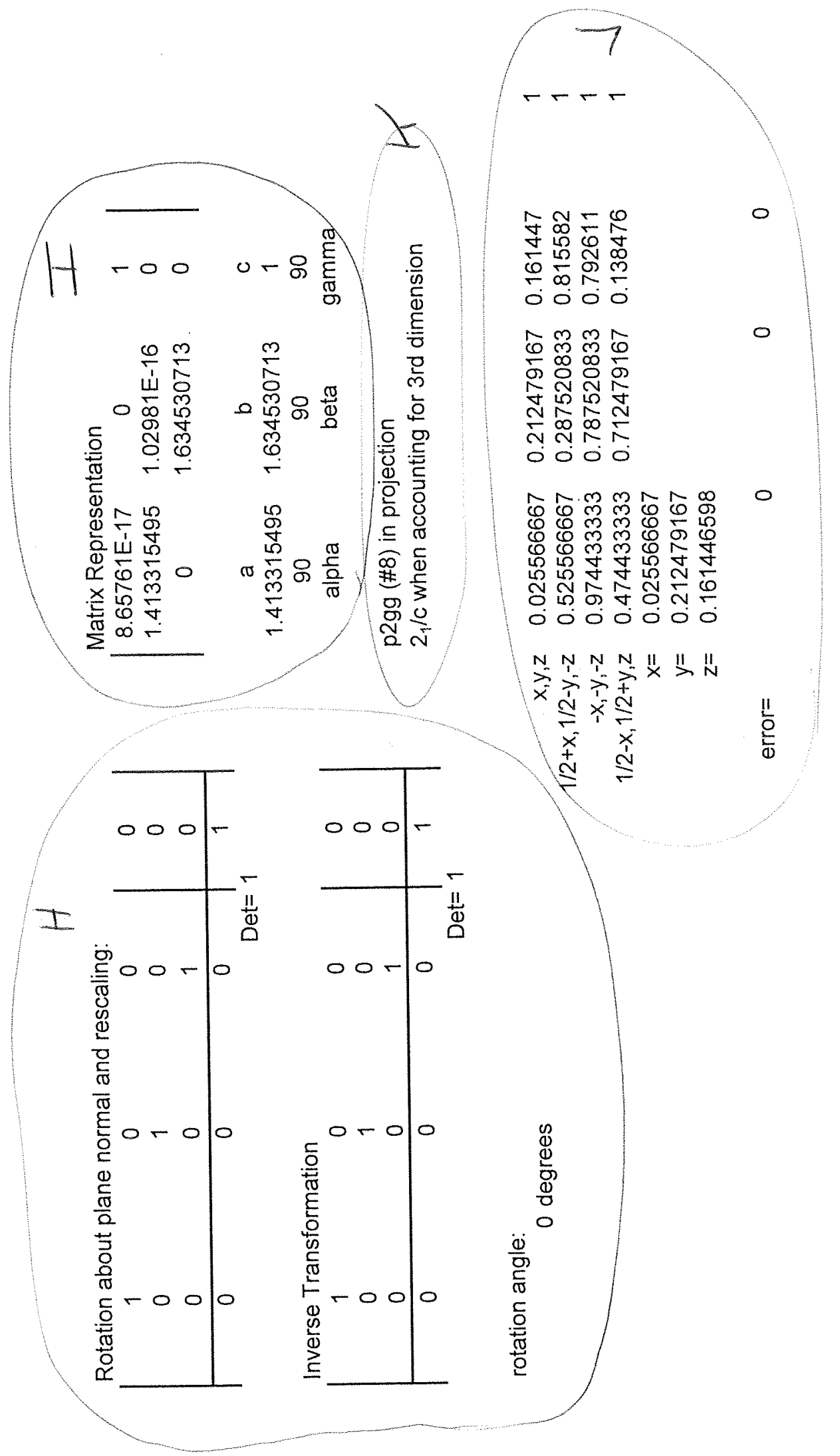



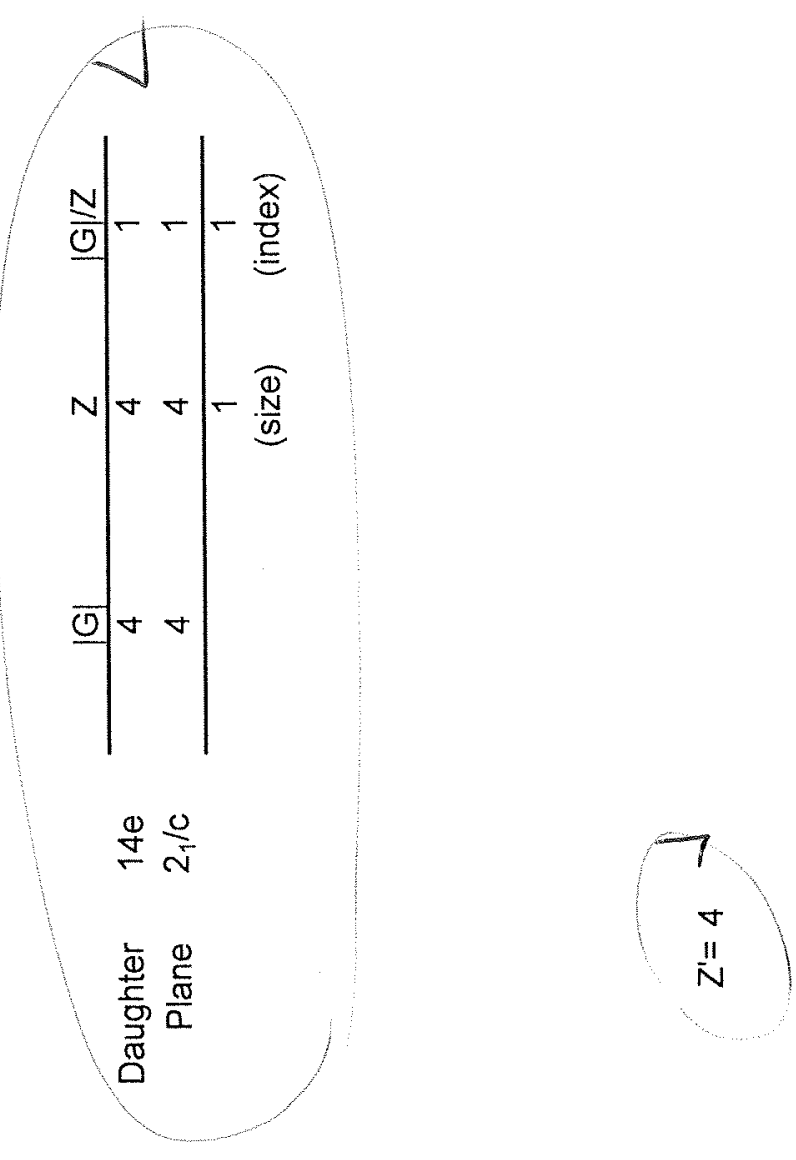


\section{Dimer Packing}

See CARBTC on pages 10 and 11 . Notes refer to the hand-written letters on the following pages.

A-E. See corresponding notes for MEZDIE01. For this structure, the histogram indicates that each molecule has a single nearest neighbor. Therefore, the molecules form dimers with Cs point group symmetry.

F. The molecular centers-of-mass reside at Wyckoff point e with point symmetry 1 . Fractional coordinates are provided.

G. The indicated transformation sorts the unit cell lengths in order to conform to reduced basis conventions. The inverse transformation is used to compute fractional center-ofmass coordinates in the transformed frame.

$\mathrm{H}$. The dot product of the matrix representation (E above) and the upper-left transformation submatrix ( $\mathrm{G}$ above) yields the transformed matrix representation. Unit cell parameters are calculated using Euclidean norms and the cosine law.

I. The Niggli matrix, normalized using the (new) a-axis length is provided.

J. The Niggli matrix conforms to the "Main Conditions" for a Type II reduced unit cell. Comparing the Niggli matrix to tabulated character definitions reveals that the reduced cell is monoclinic (character 35), but close to C-centered-orthorhombic (oC, character 23).

$\mathrm{K}$. Transforming the fractional center-of-mass coordinates $(\mathrm{F})$ using the inverse transformation matrix $(\mathrm{G})$ yields the indicated coordinates. There are four distinct molecules in the primitive cell $\left(Z^{\prime}=4\right)$.

L. Since cell reduction $(\mathrm{G}-\mathrm{K})$ did not yield a simpler reference lattice, and recognizing the single nearest neighbor, consider the centers-of-mass for dimers.

M. The dimer centers-of-mass reside at Wyckoff point a with point symmetry -1 . Fractional coordinates are provided.

$\mathrm{N}$. The indicated transformation halves the volume of the original unit cell (with matrix representation provided in $\mathrm{E}$ above) and rotates the unit cell. No origin shift is necessary in this case. Halving the volume of the cell recognizes that the center-of-mass lattice has a single dimer in the primitive unit cell.

O. Applying the upper-left submatrix of the transformation to the original matrix representation yields the transformed matrix representation. Unit cell parameters are calculated using Euclidean norms and the cosine law.

P. The Niggli matrix, normalized using the (new) a-axis length is provided.

Q. The Niggli matrix conforms to the "Main Conditions" for a Type I reduced unit cell. Comparing the Niggli matrix to tabulated character definitions reveals that the reduced cell is $\mathrm{C}$-centered monoclinic (character 10), but close to rhombohedral (hR, character 9).

R. Transforming the fractional center-of-mass coordinates for the dimers (M) using the inverse transformation matrix $(\mathrm{N})$ yields the indicated coordinates. There is one dimer in the primitive cell $\left(Z^{\prime}=1\right)$. The dimer center-of-mass is located at Wyckoff point a of rhombohedral space group R-3m (no 166).

$\mathrm{S}$. The symmetry-breaking transformation from the reference lattice containing orientationally disordered dimers to a monoclinic cell with disordered dimers has size one 
and index 3. Orientationally ordering the dimers is a subsequent transformation with size 4 and index 4.

T. The tabulated transformation from a reduced basis in character 9 to the conventional cell (using the hexagonal setting for the rhombohedral cell) is indicated.

U. The transformation from the reference lattice to the observed cell is given by the inverse of the product of the transformations in $\mathrm{N}$ and $\mathrm{T}$ above. This overall transformation matrix is provided. COPL indicates that the overall transformation matrix is consistent with the indicated symmetry-breaking transition with order parameter F2+. 


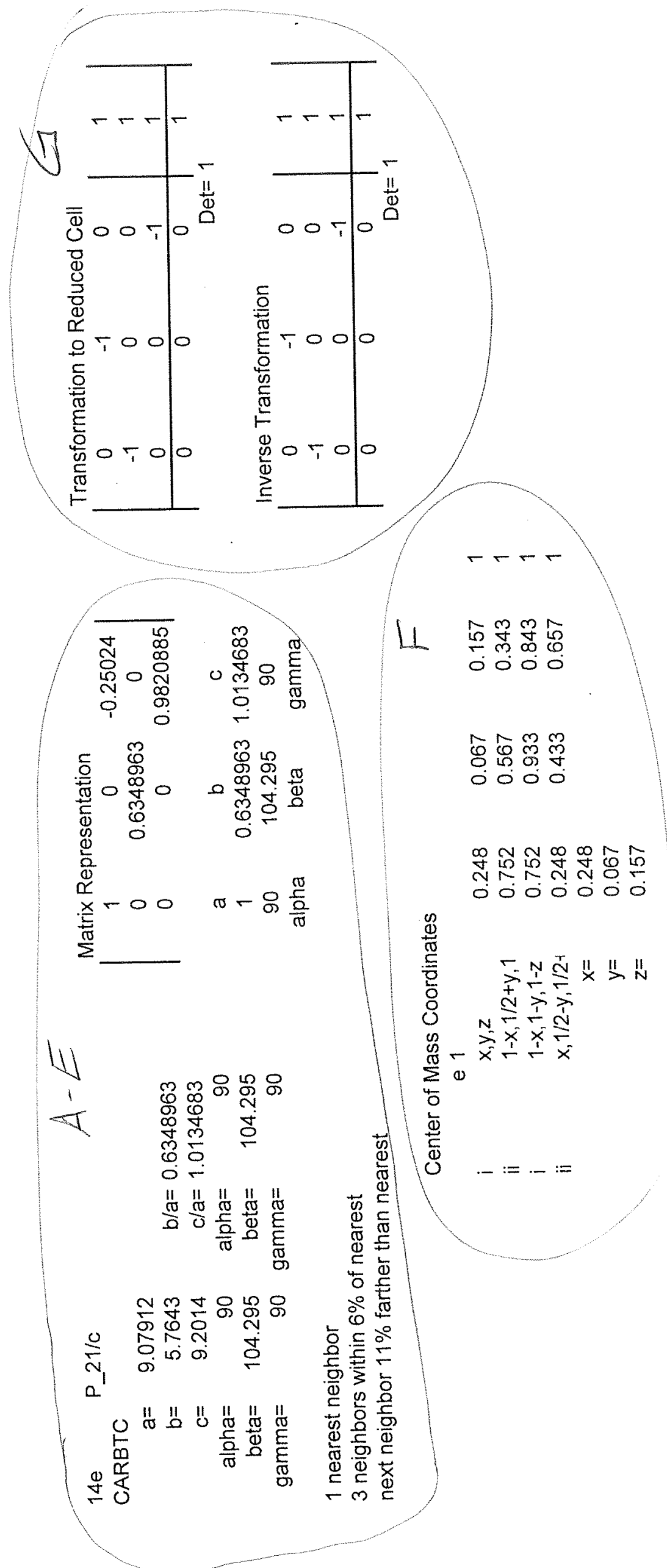




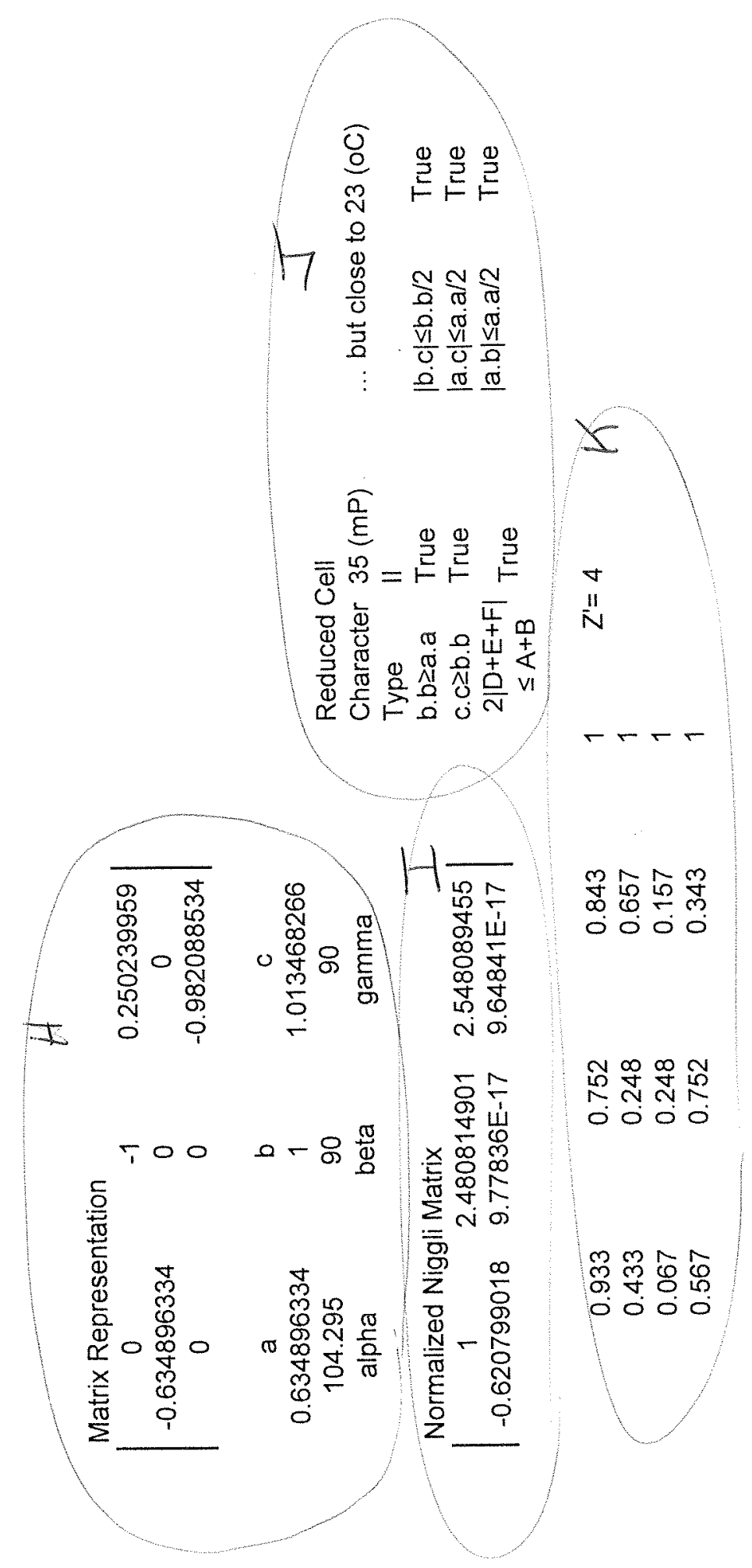



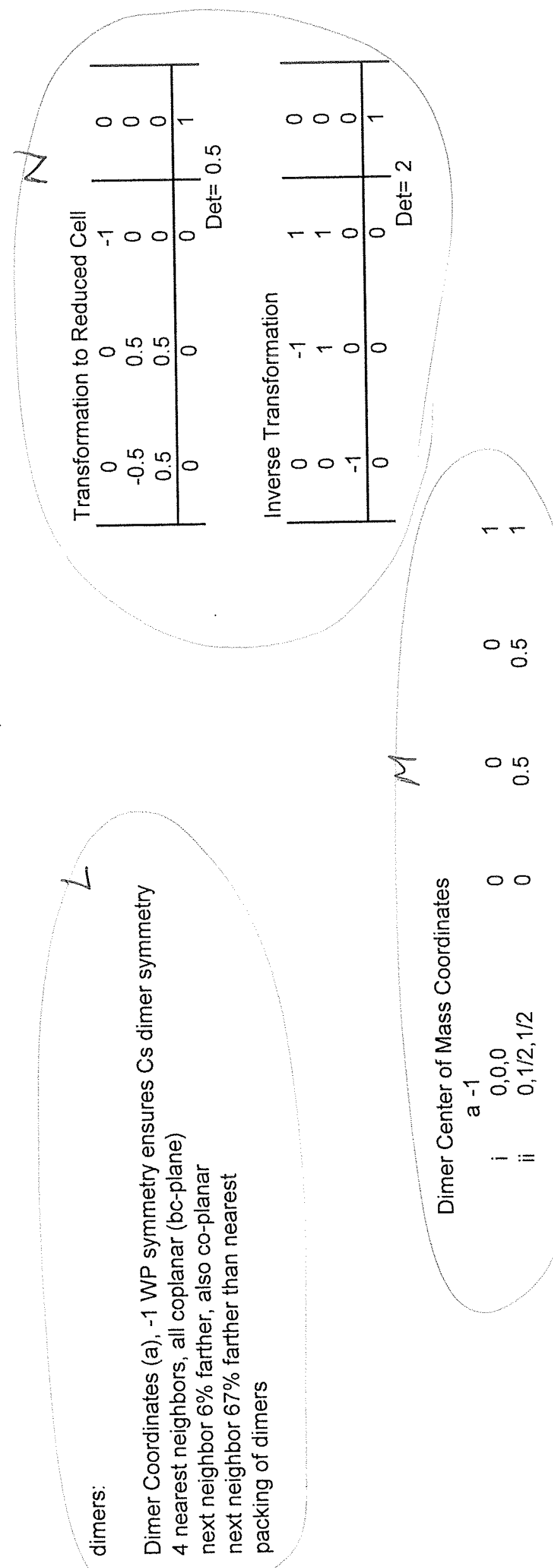


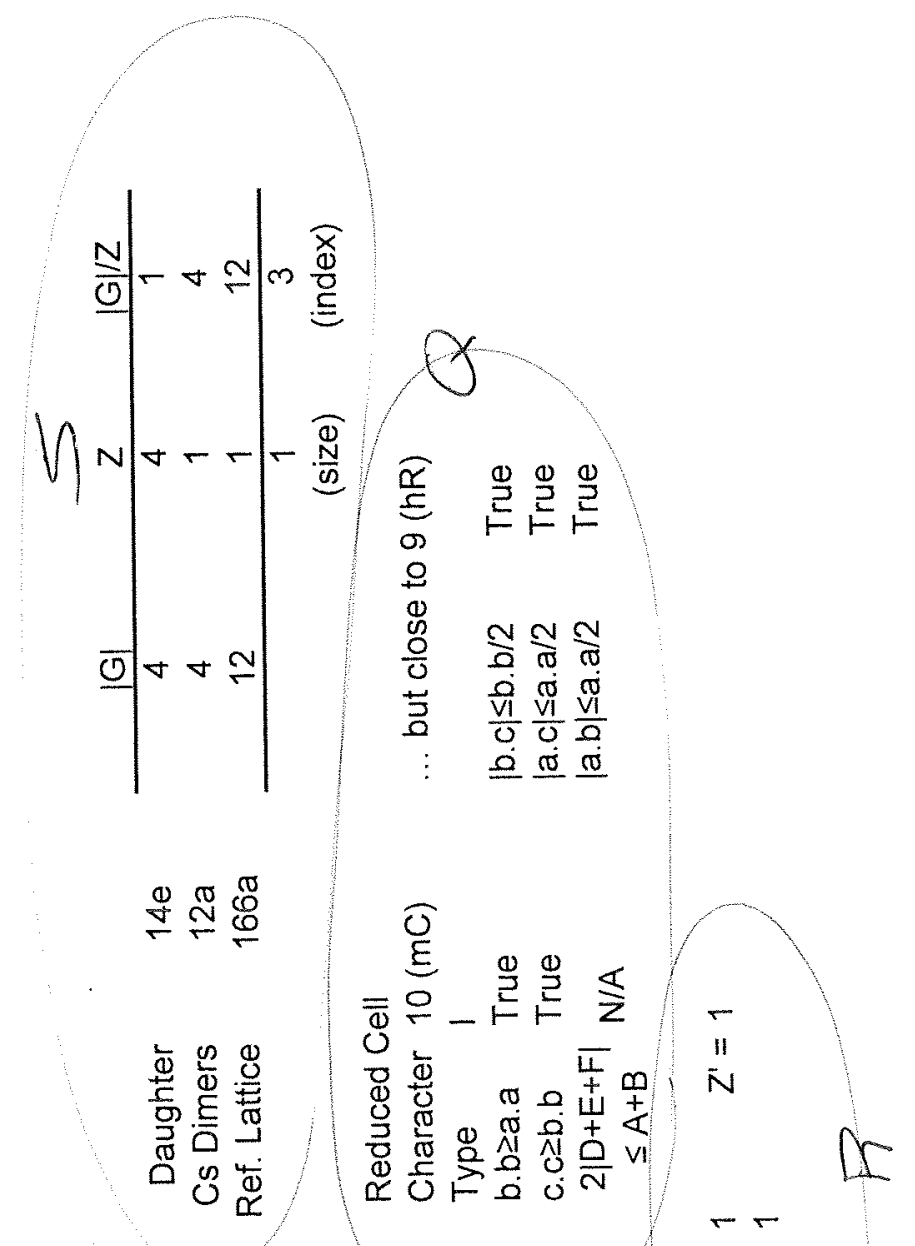

0

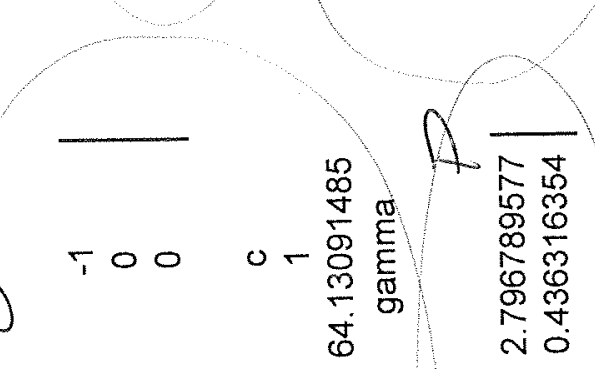

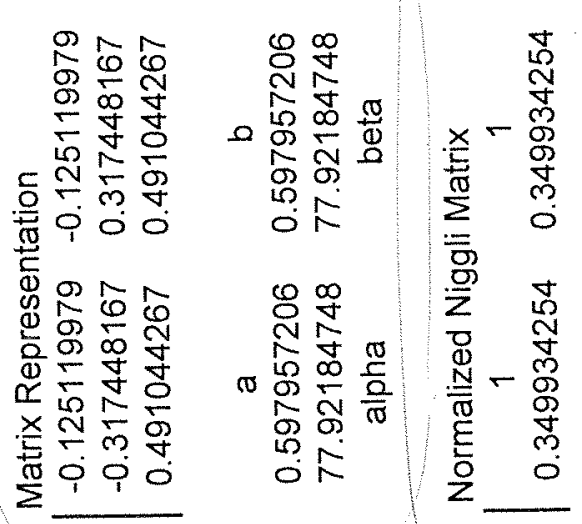

or

00

응 


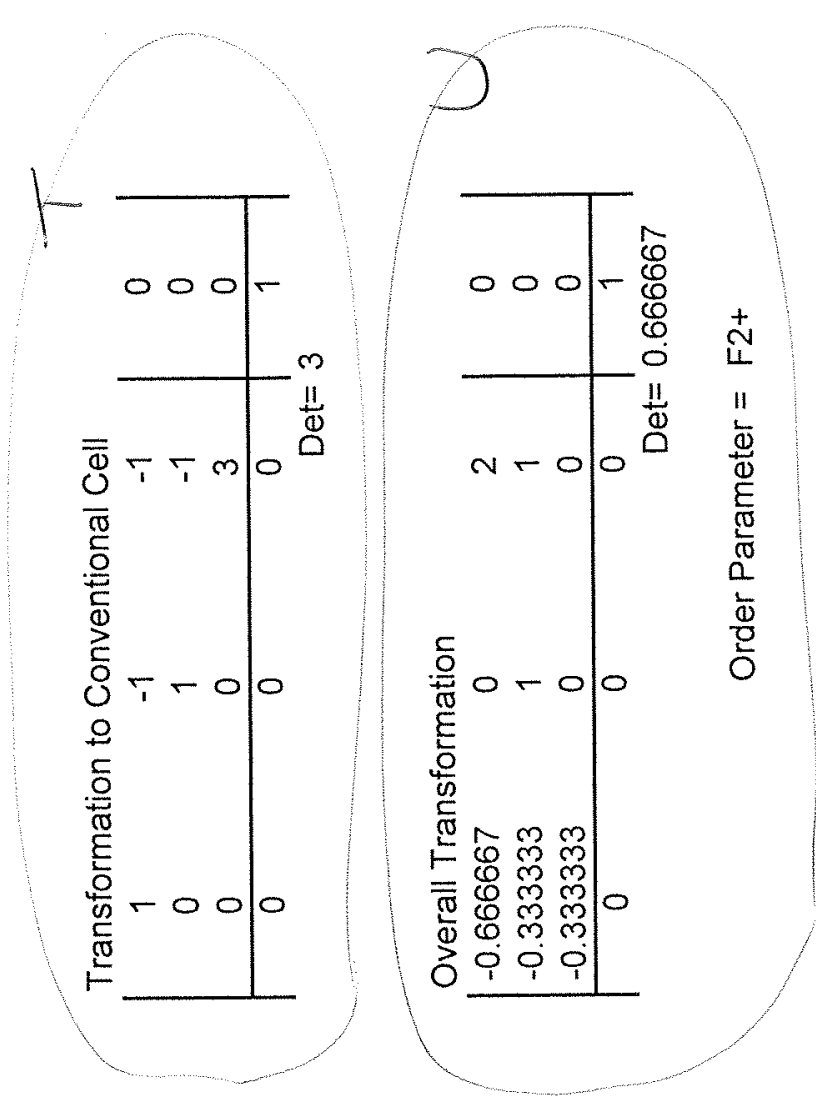




\section{Mixed Packing}

See KOXKOX and SENLAY on page 1. Notes refer to the hand-written letters on the following pages.

A-E. See corresponding notes for MEZDIE01. For this structure, the histogram indicates that each molecule has three nearest neighbors forming two-dimensional planes. The planes are shown to have $\mathrm{p} 2 \mathrm{gg}$ projection symmetry.

F. Two molecules in the unit cell reside at Wyckoff point a with point symmetry $-43 \mathrm{~m}$. These molecules have 12 nearest neighbors. The remaining six molecules in the unit cell reside at Wyckoff point $\mathrm{c}$ with point symmetry -4 . They have 2 nearest neighbors. Fractional coordinates are provided.

G. Since the unit cell is already a reduced basis, there is no need for an additional transformation. The Identity matrix leaves the cell and the center-of-mass coordinates unchanged.

$\mathrm{H}$. The cell is a cube which belongs to primitive cubic $(\mathrm{cP})$ character 3 .

I. The center-of-mass coordinates are consistent with Wyckoff orbits a and $\mathrm{c}$ of space group 223. In this case, the reference lattice has a higher symmetry than the observed structure solely due to the orientations of the molecules. There is no deformation of the cell nor translation of the centers-of-mass within the cell.

J. The symmetry breaking transition in passing from the reference lattice to the observed (daughter) cell has size 1 and index 2.

$\mathrm{K}$. There is no transformation needed for character 3 .

L. The overall transformation matrix from observed cell to conventional reference lattice is the identity since the component transformations ( $\mathrm{G}$ and $\mathrm{K}$ ) are identities. COPL indicates that the overall transformation matrix is consistent with the indicated symmetrybreaking transition with size 1 and index 2 as calculated in $\mathrm{J}$ above. The order parameter is at the gamma point of the Brillioun zone (GM2-). 


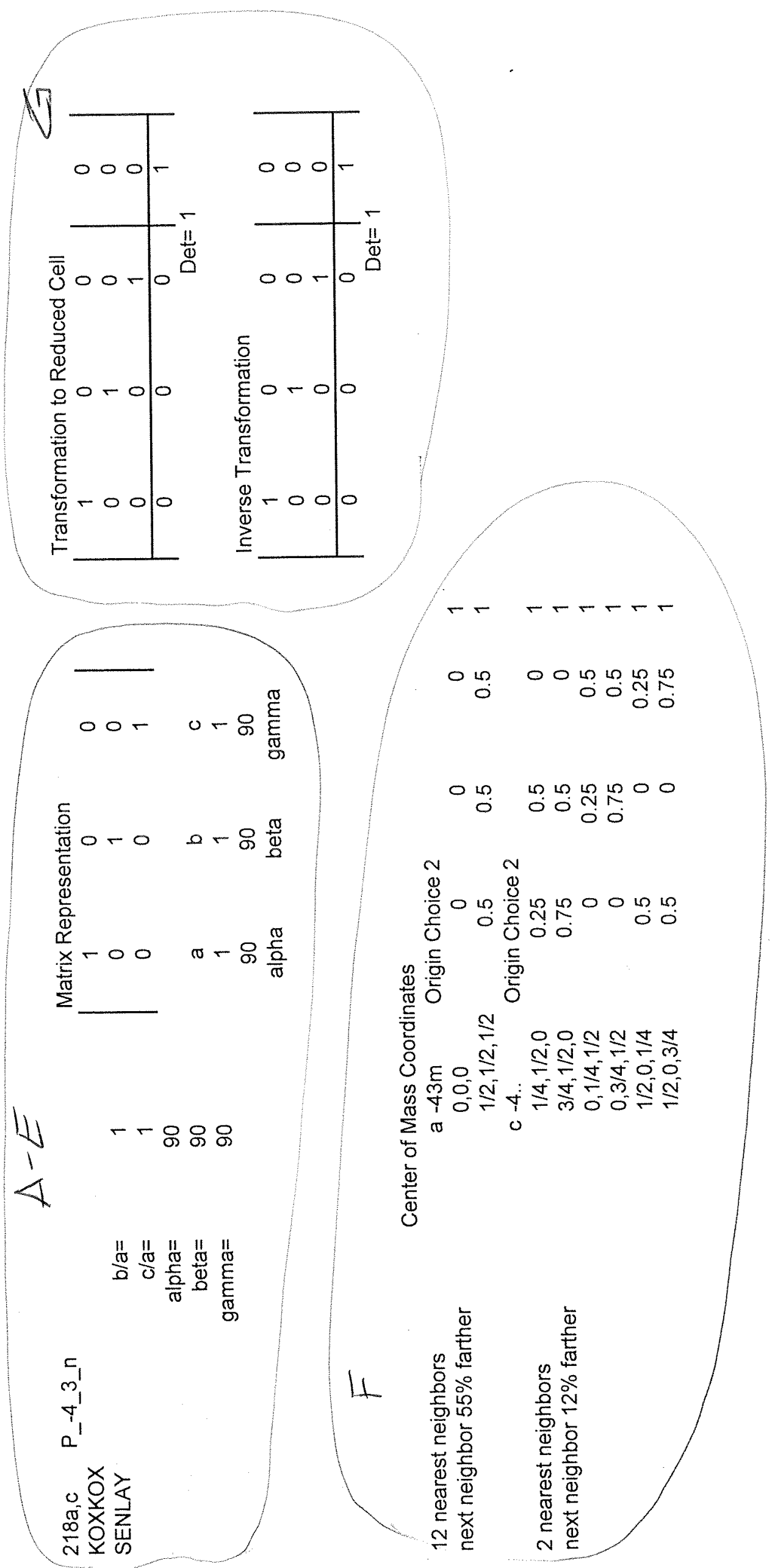




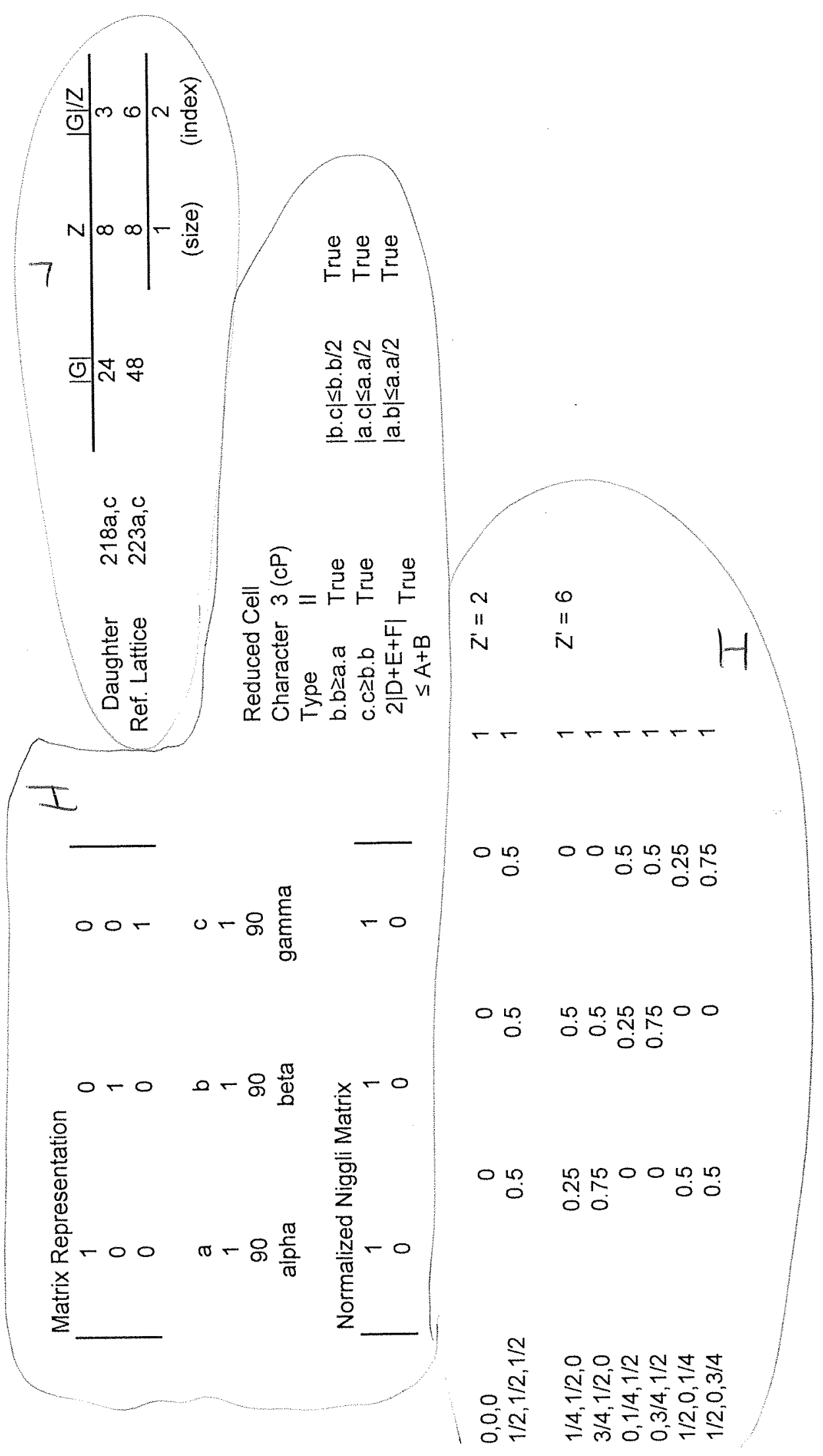




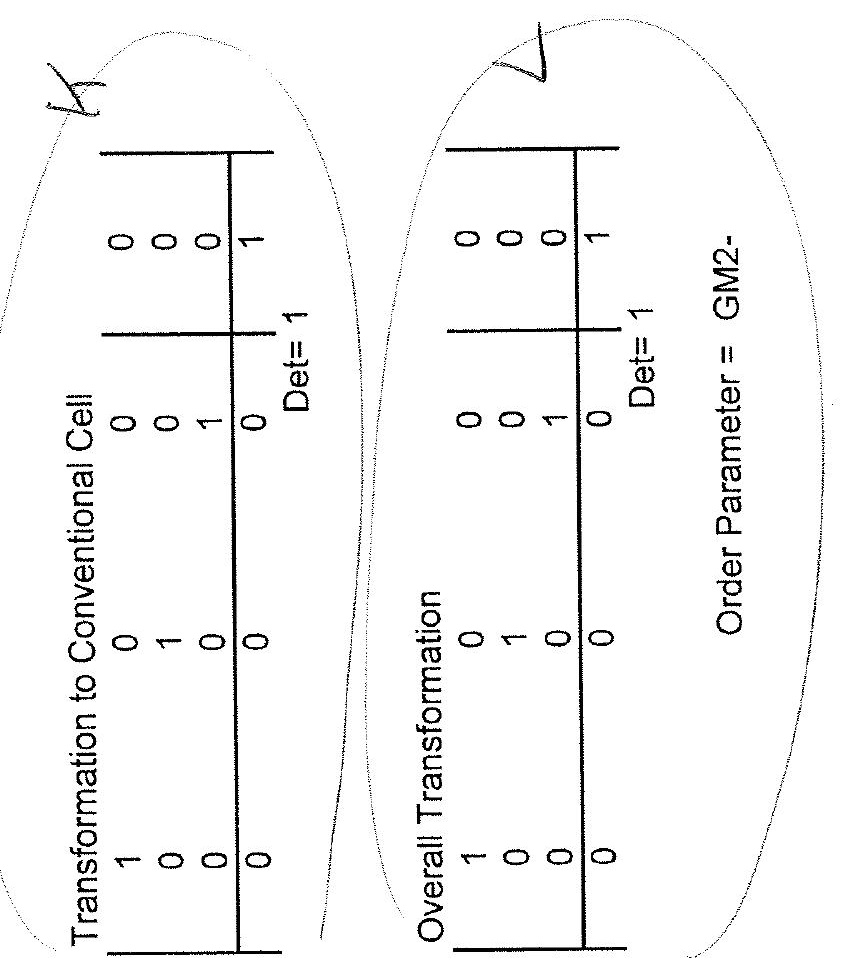



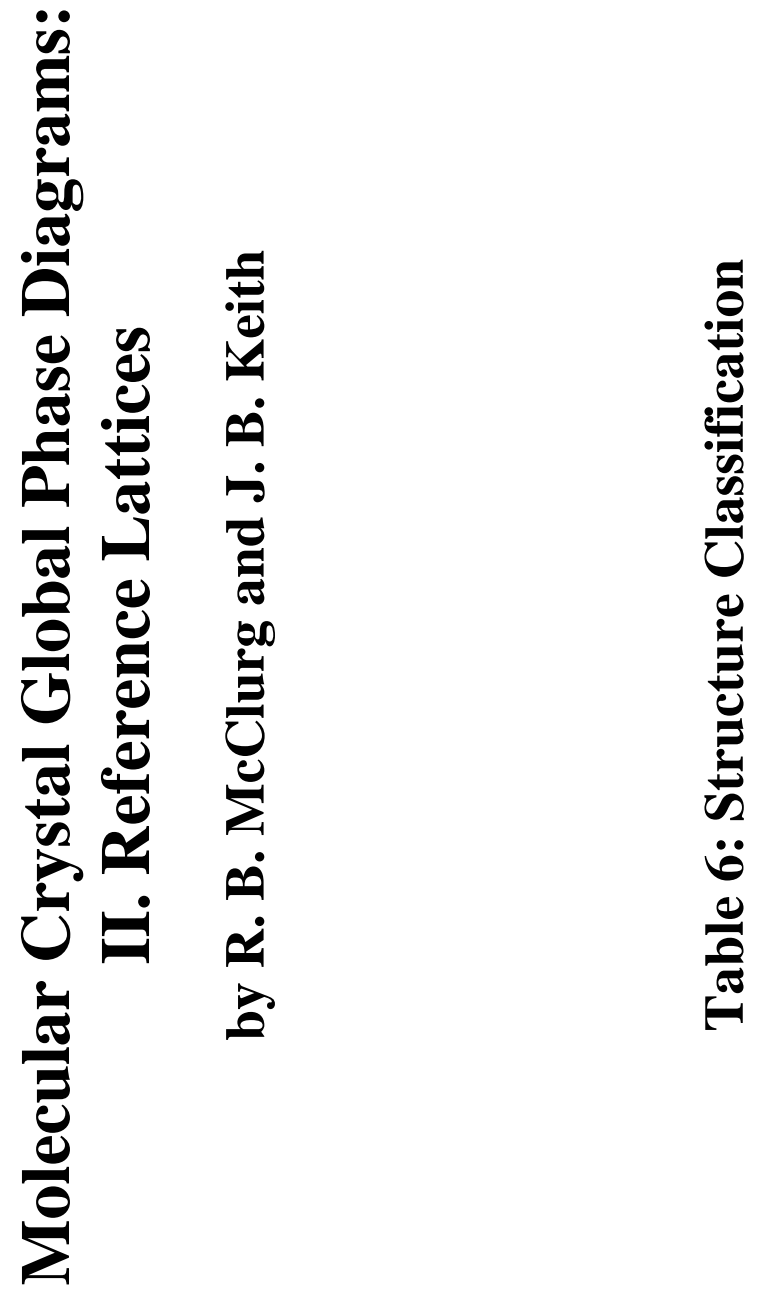

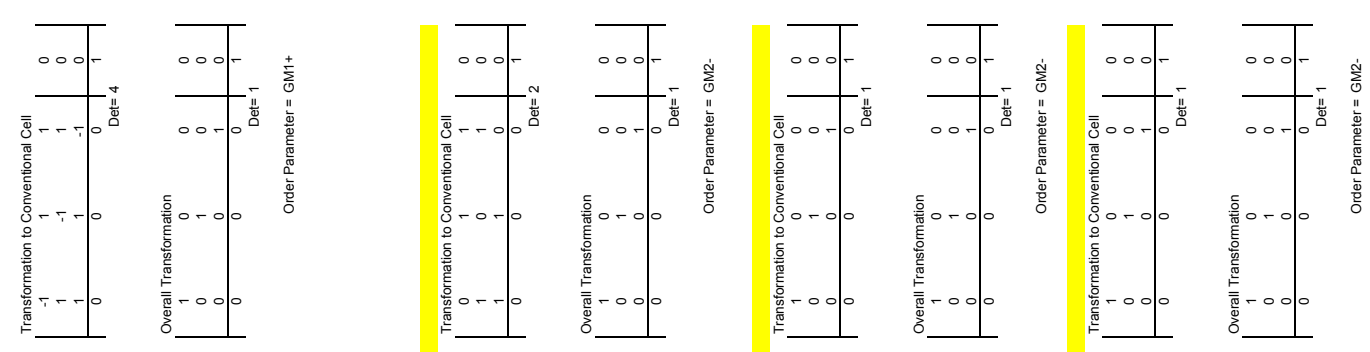

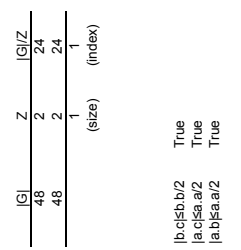

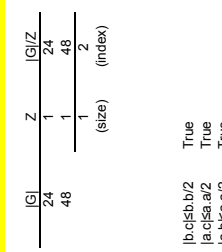

의에

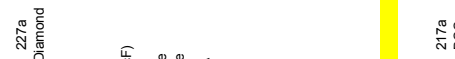

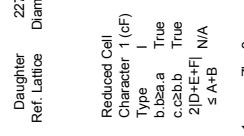

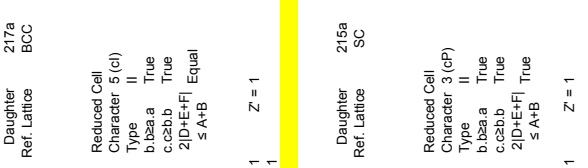

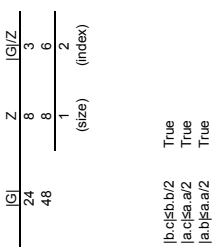

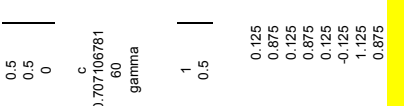

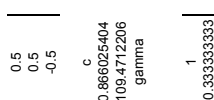

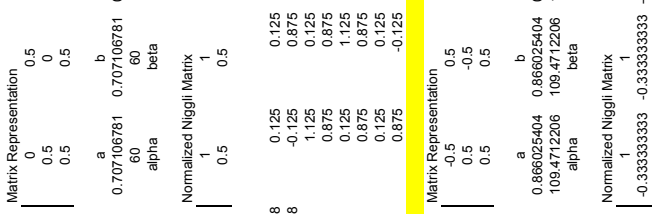

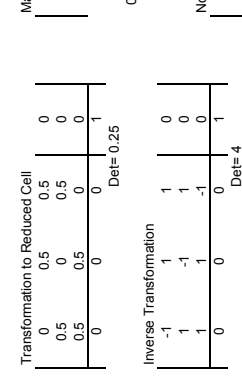

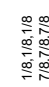

$$
\text { - }
$$

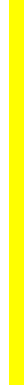

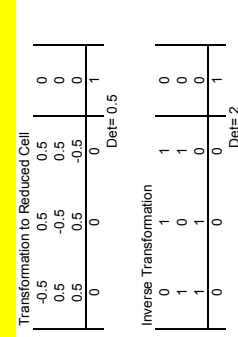

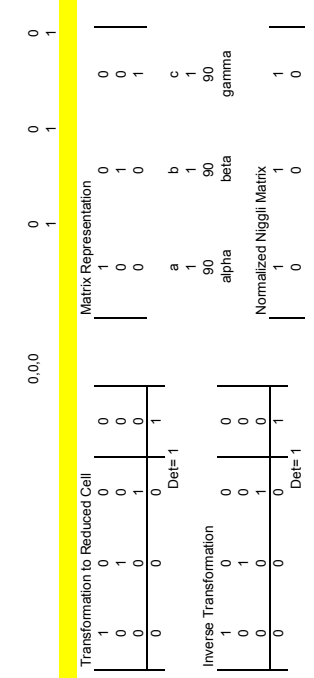

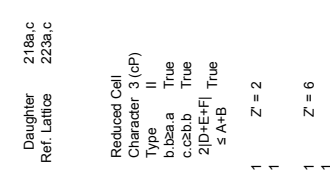

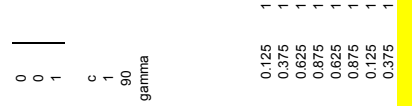

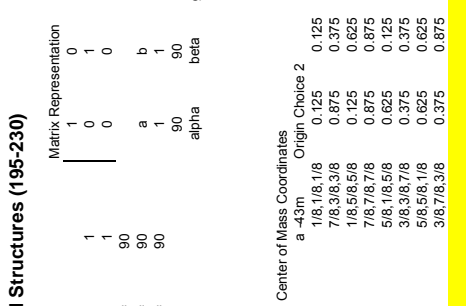

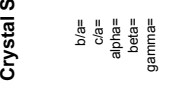

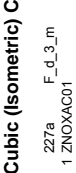

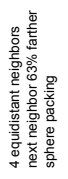

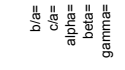
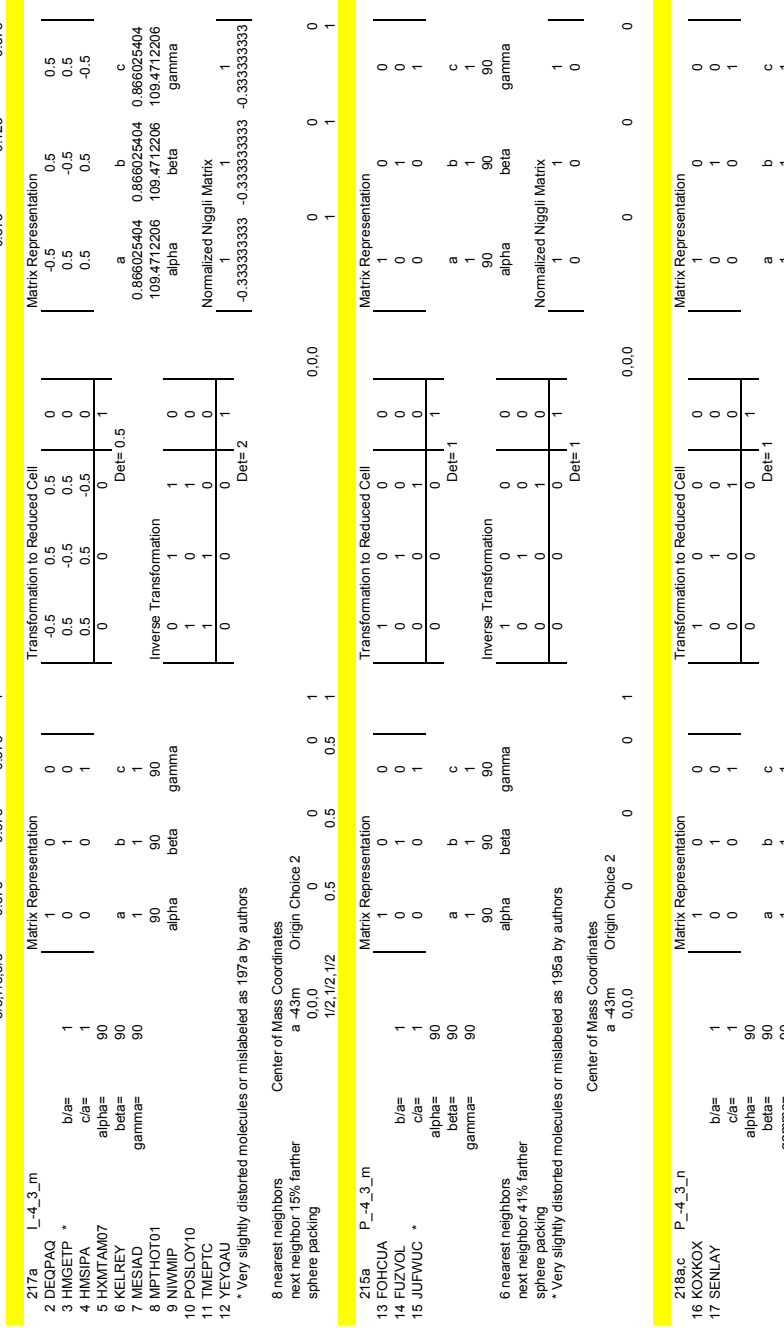

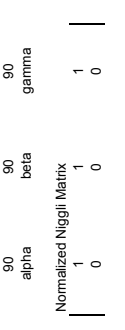

응 어맹몽몽

-

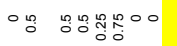

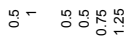

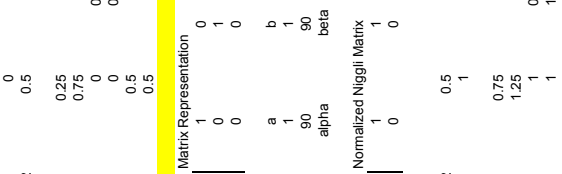

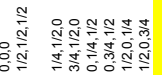

$\frac{1}{\mathrm{~N}}$

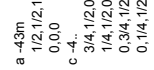
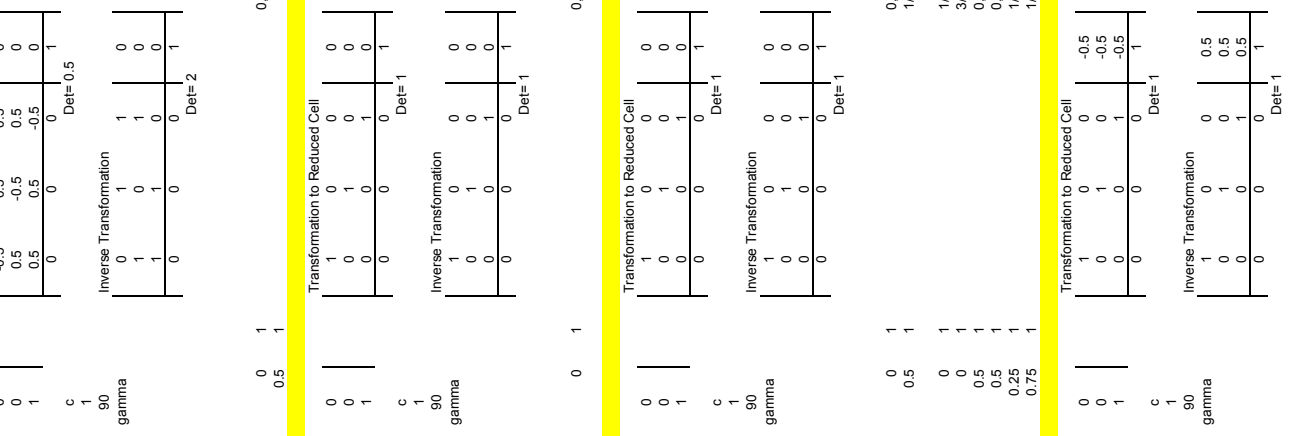

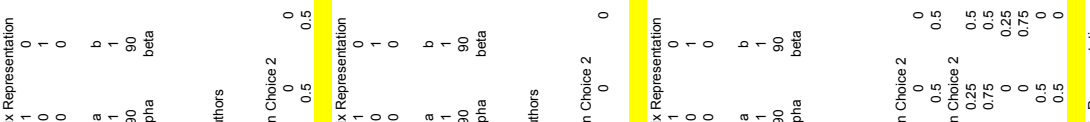

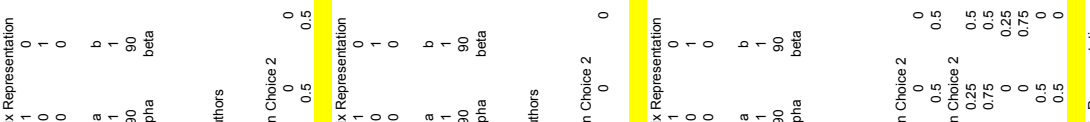

.

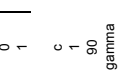

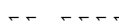

잉

ㅇํㅇ 0 땡ำ

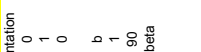

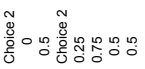

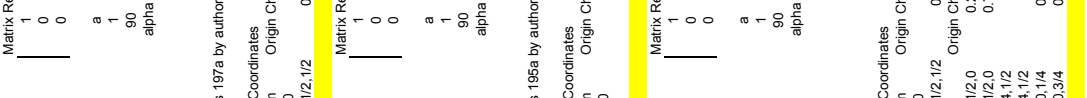

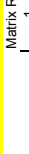

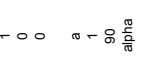

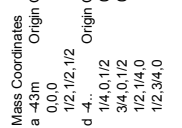

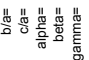

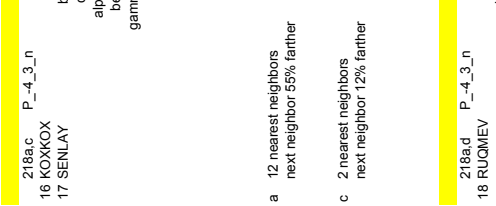

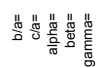

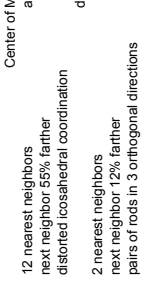




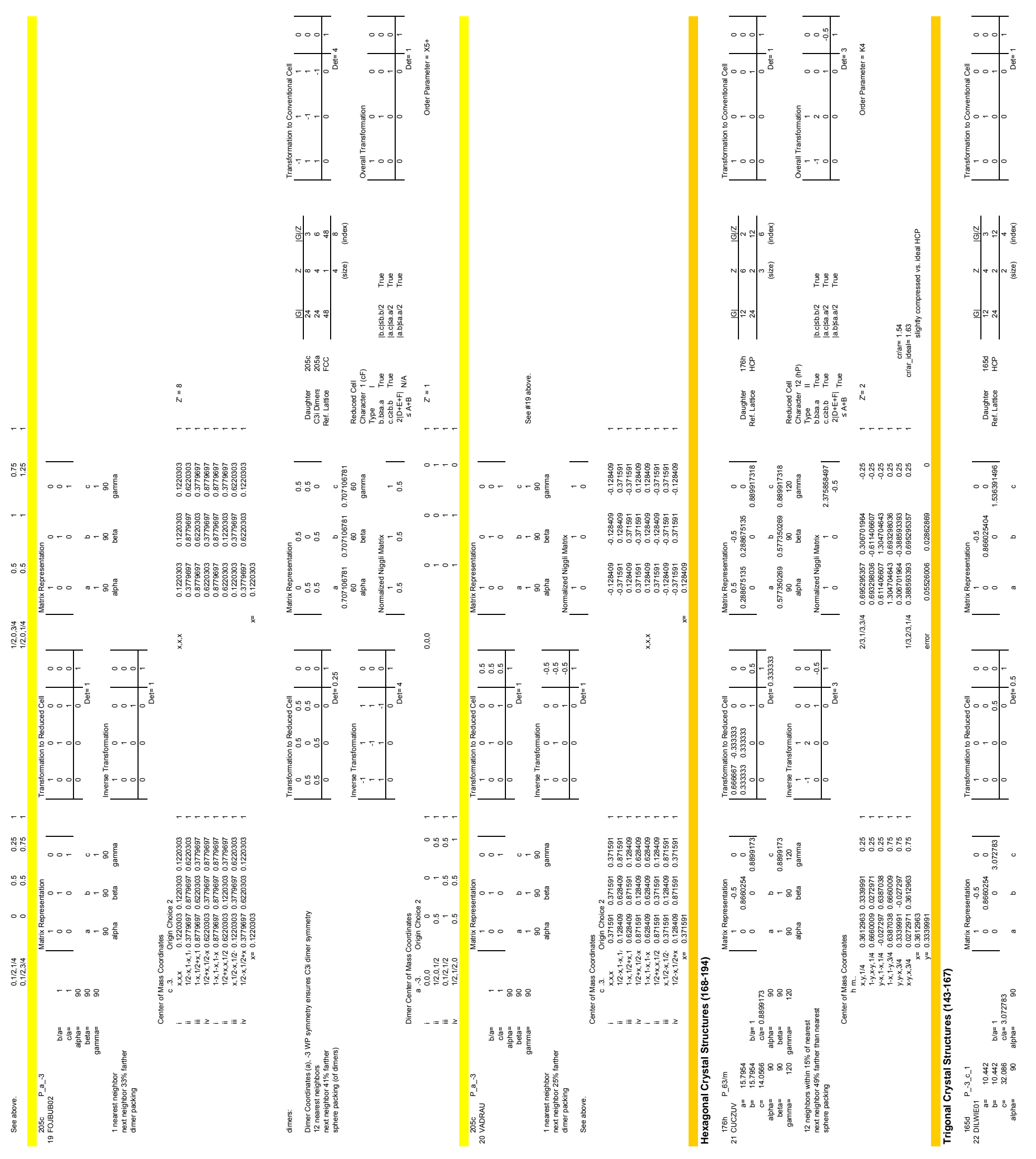



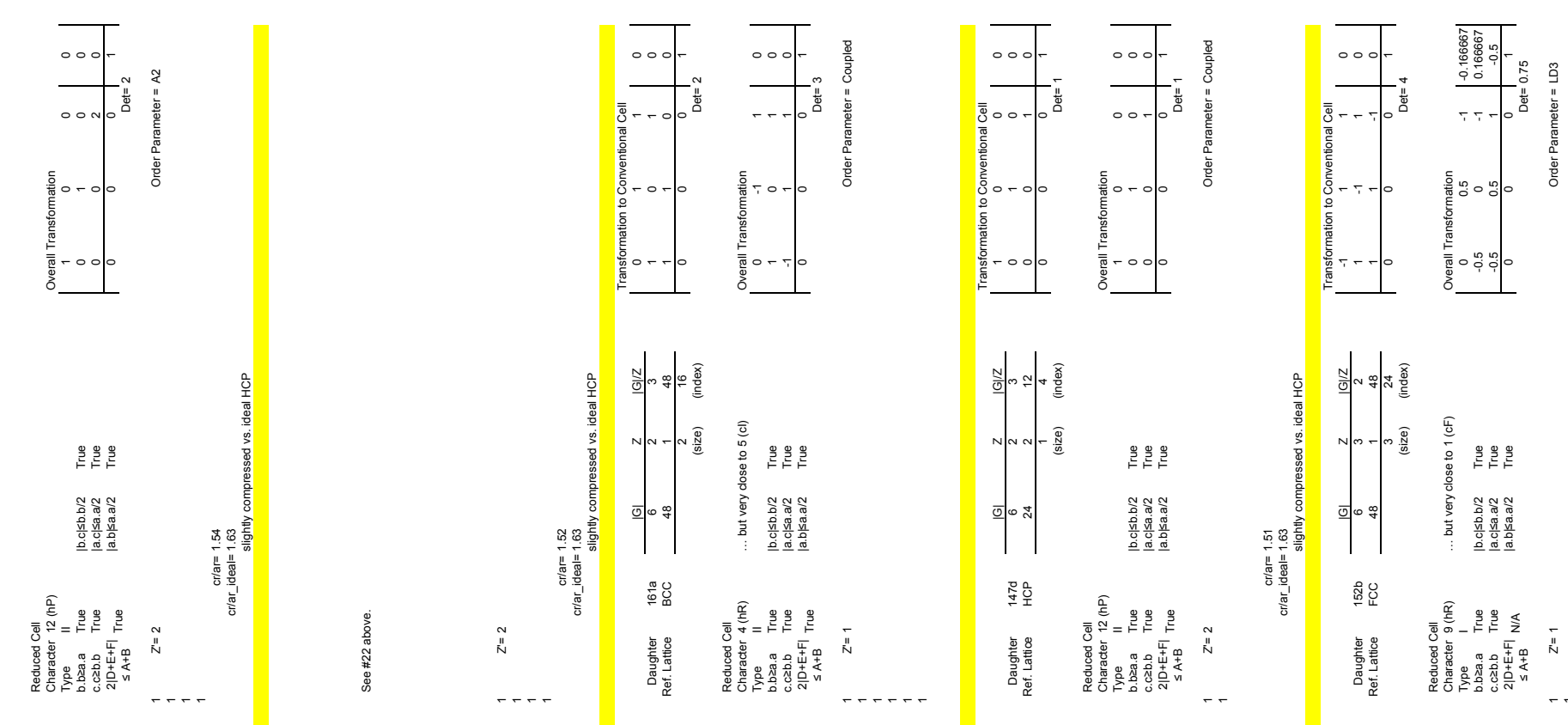

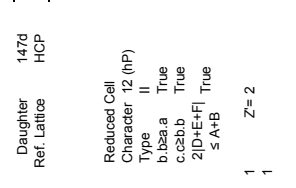

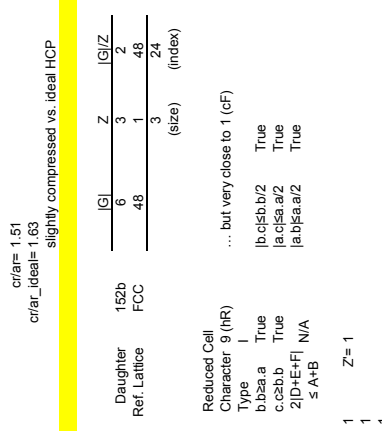

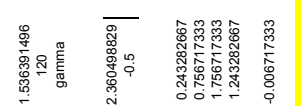

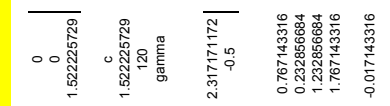

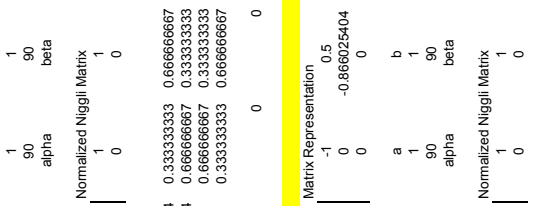
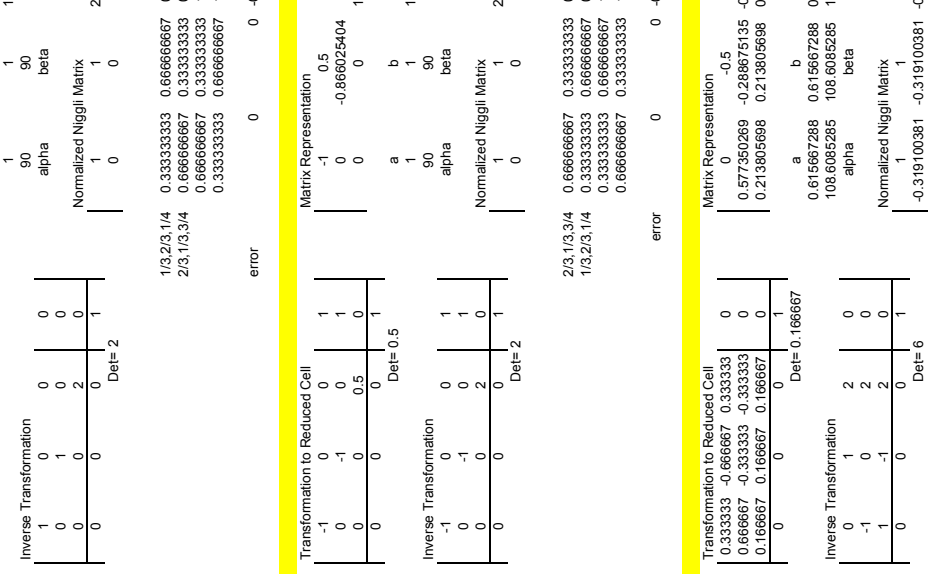

แแแ!

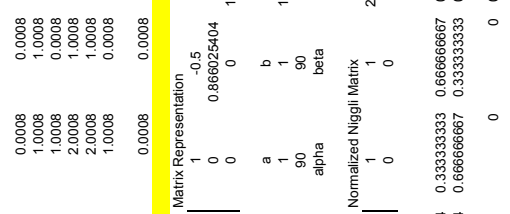

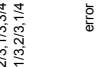
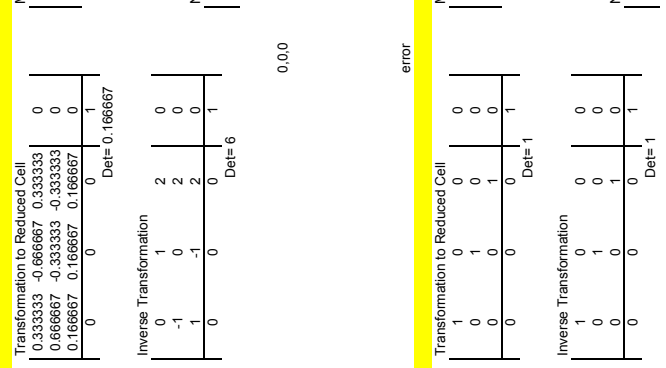

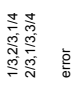
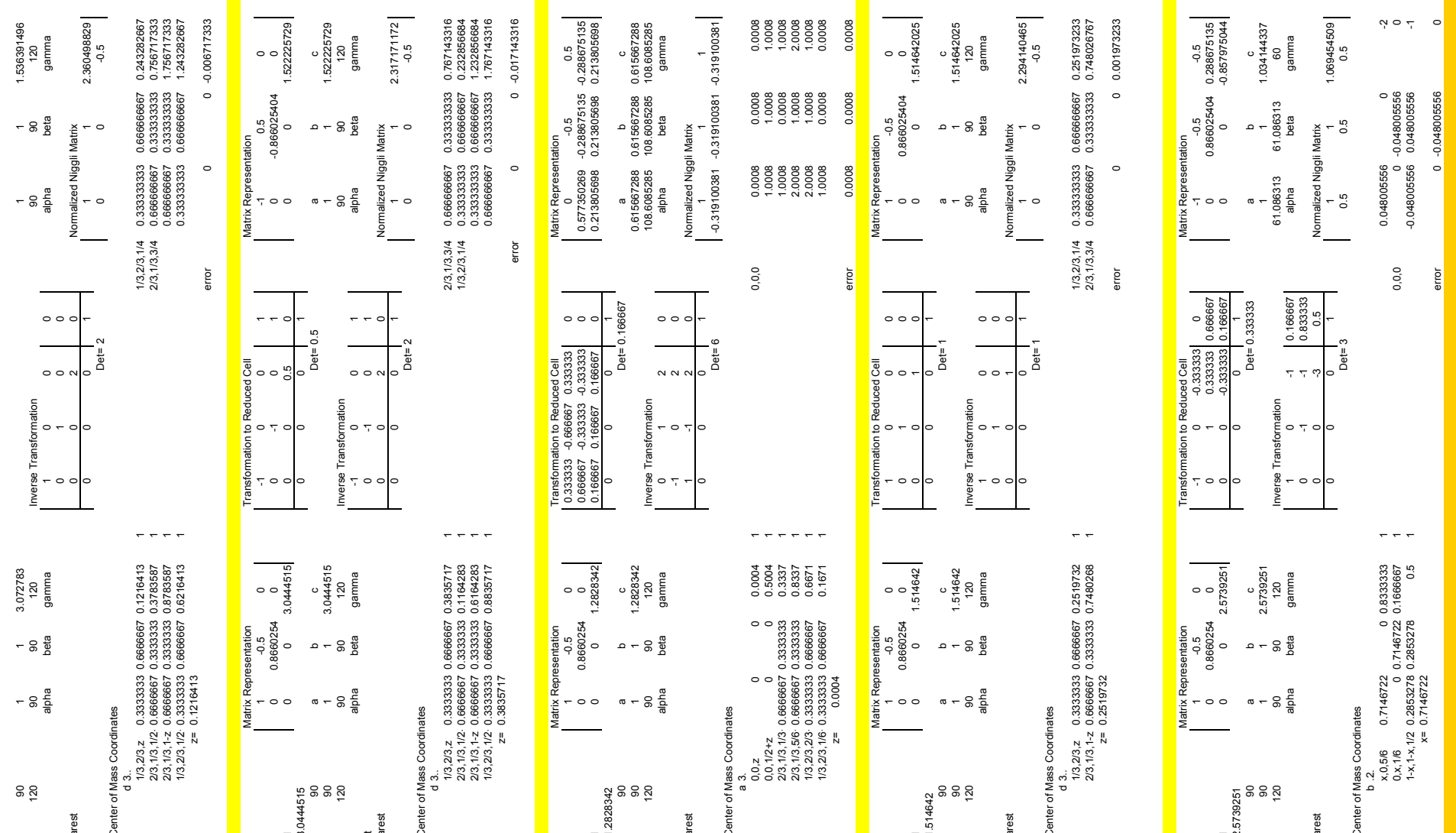

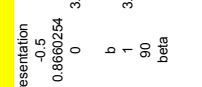
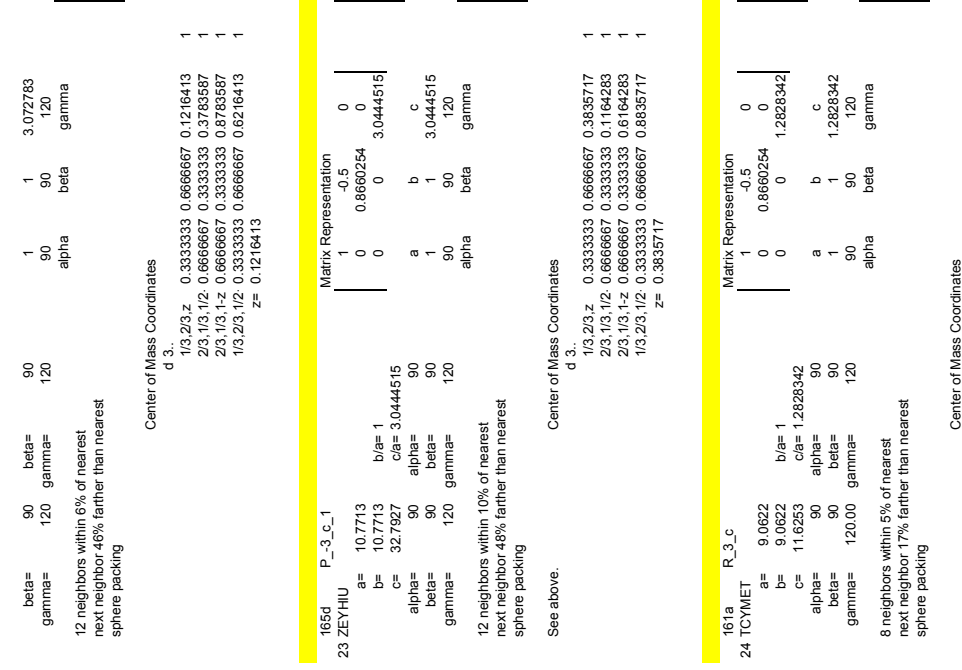

|fos.
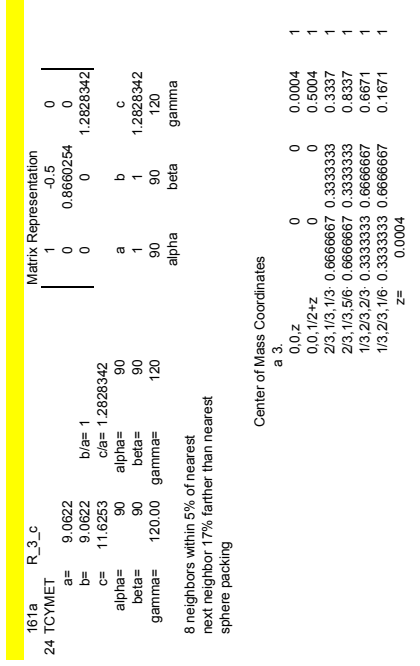

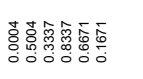

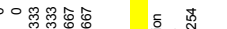
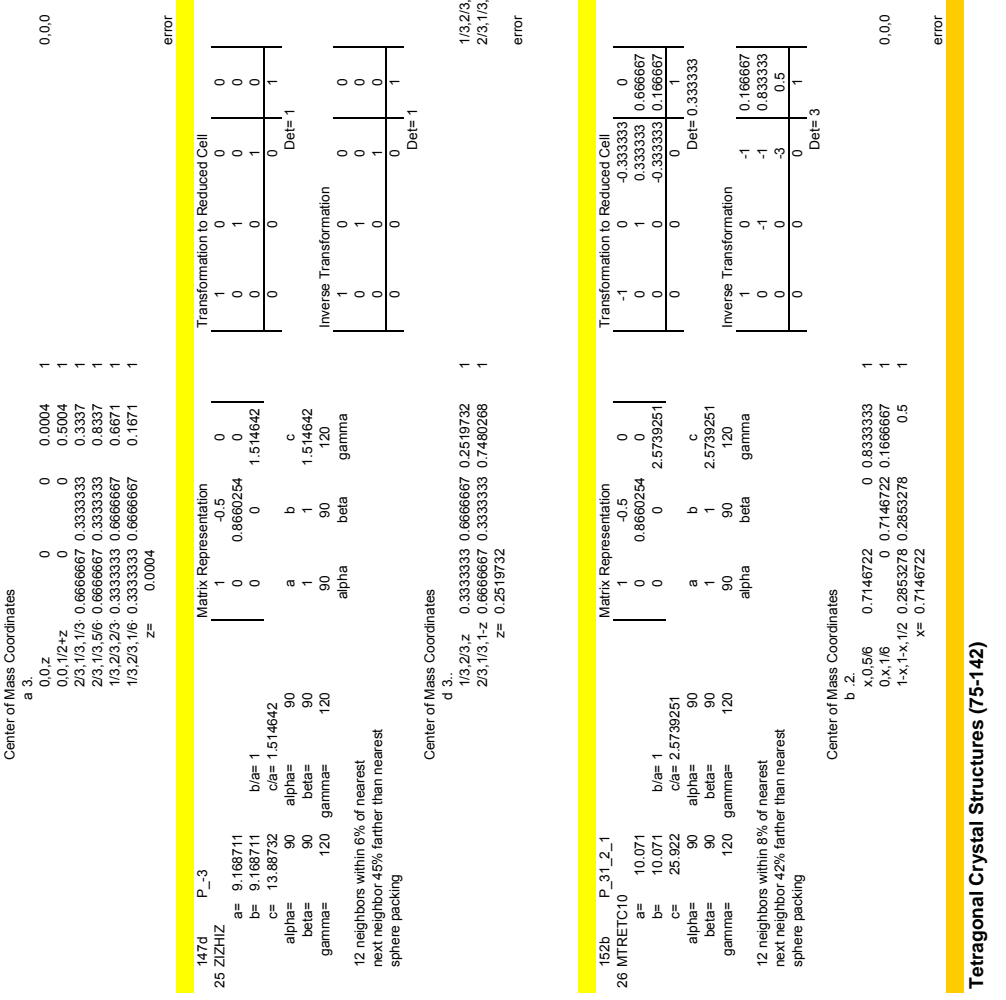


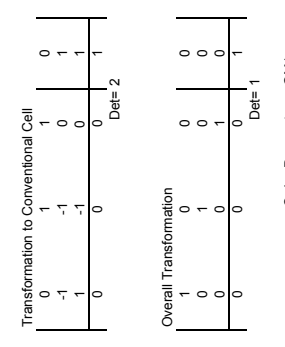

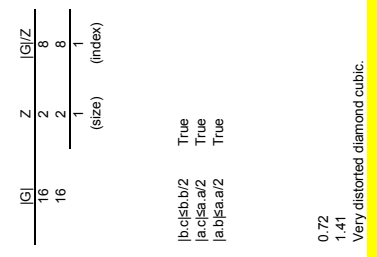

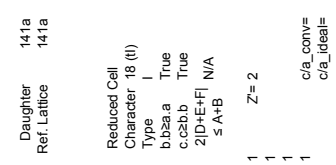

औौ

Hil $y=$
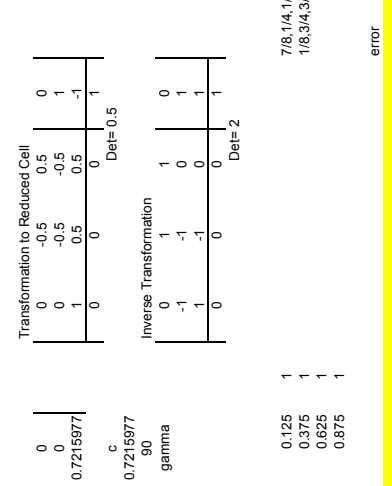

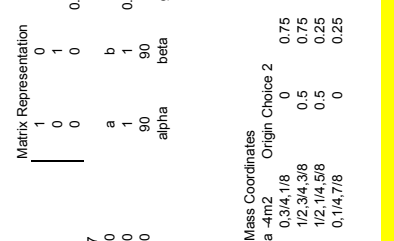

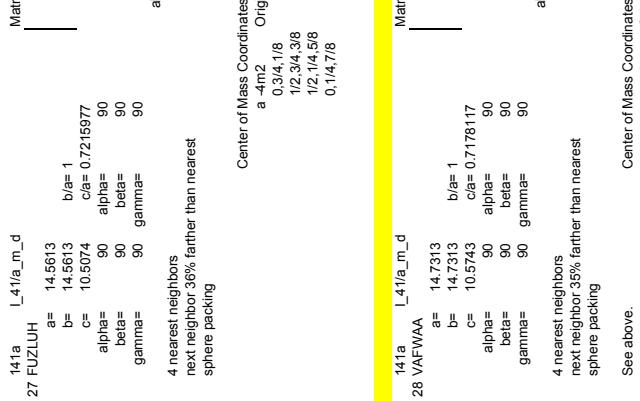

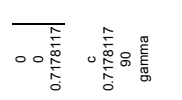

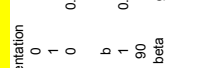

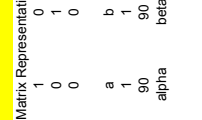

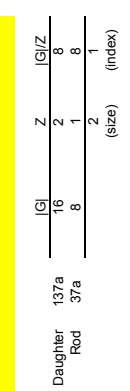

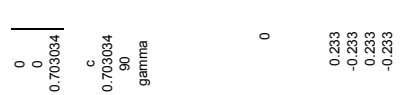

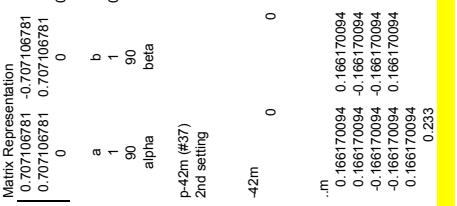

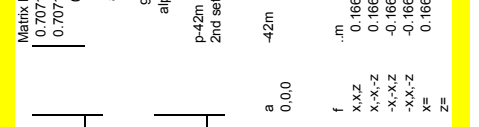
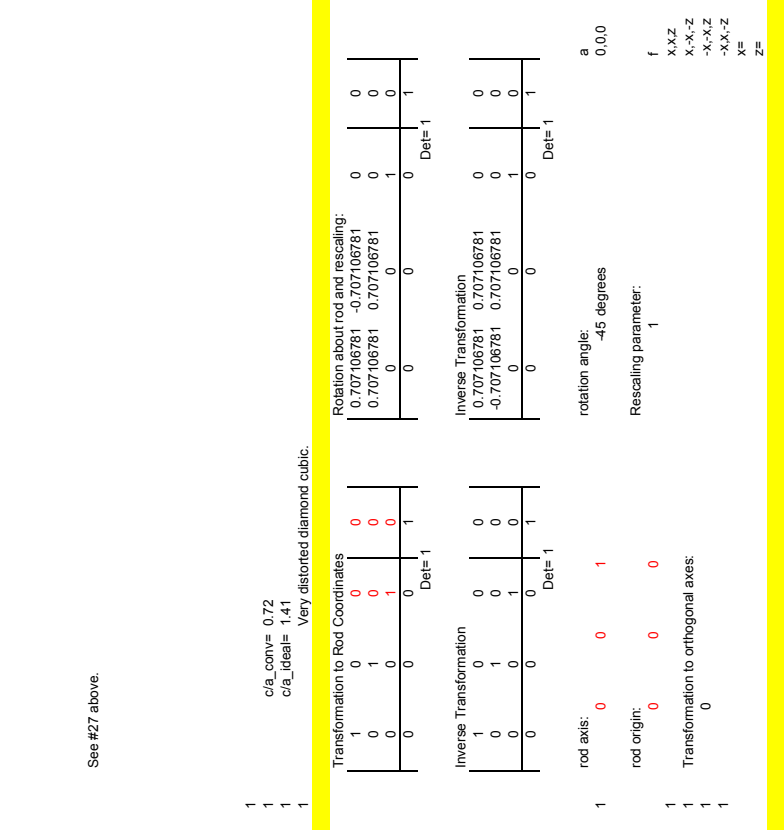

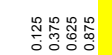

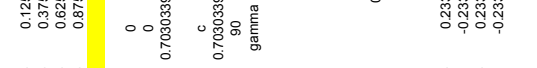

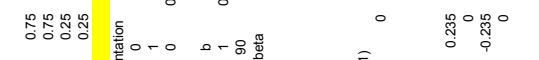

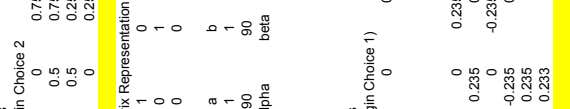

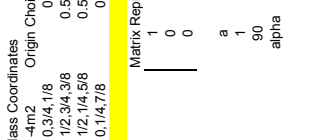

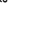

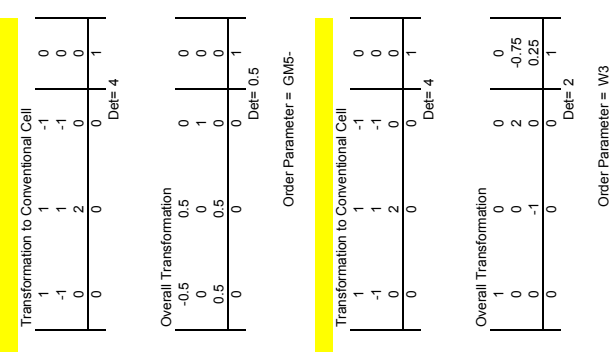

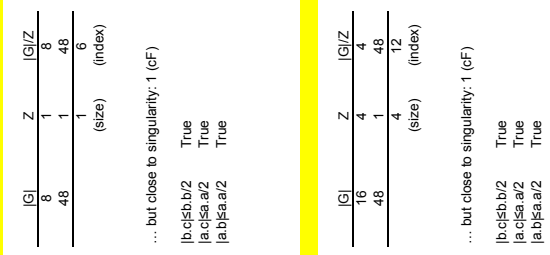

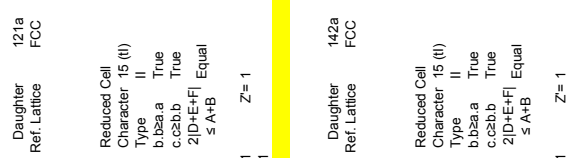

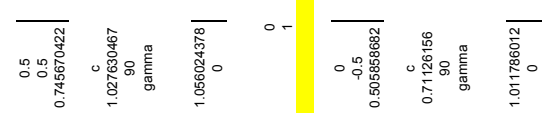

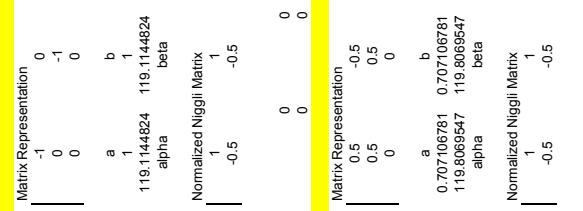
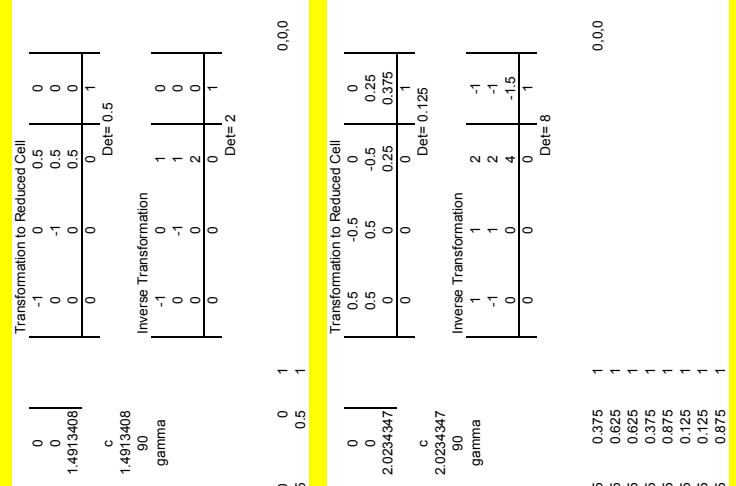

年

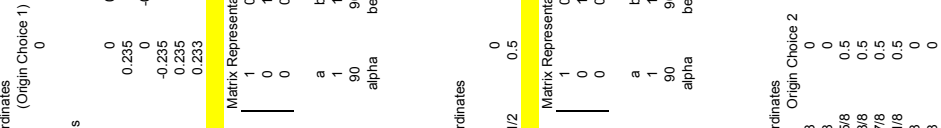

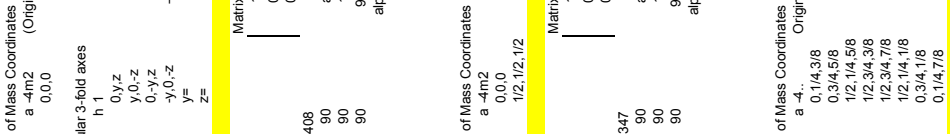

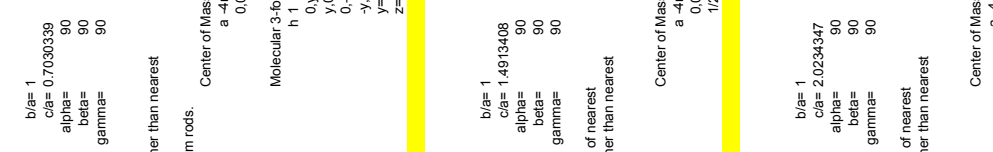

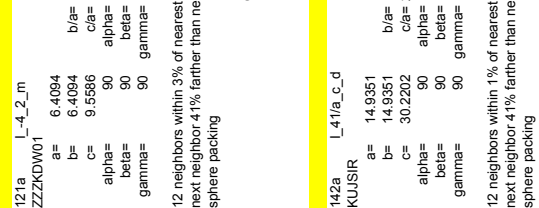

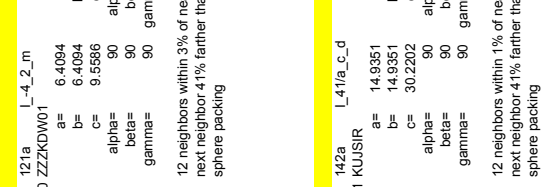



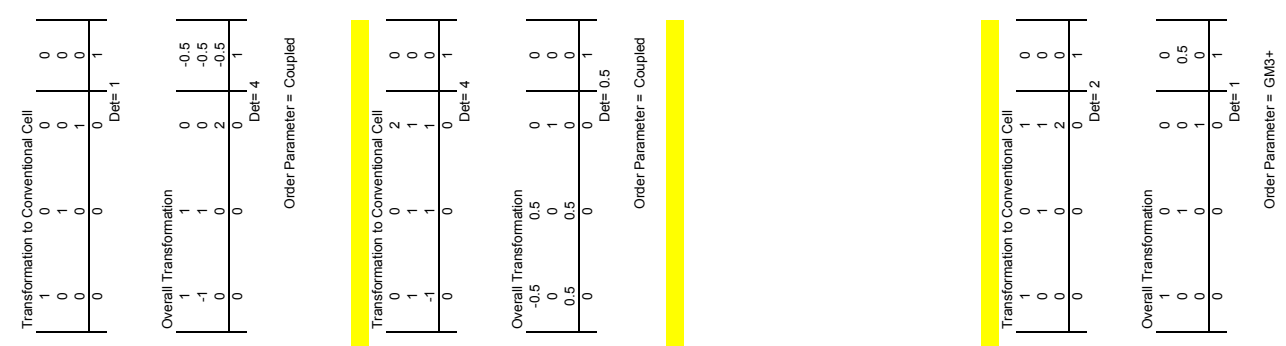

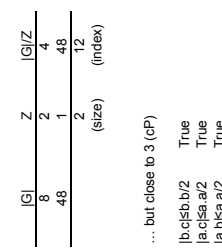

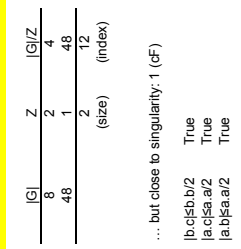

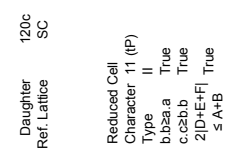

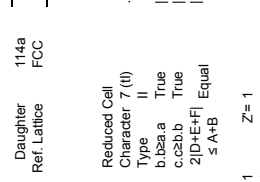

$\underset{0}{N \infty} \infty \cdots \infty \cdots \infty$
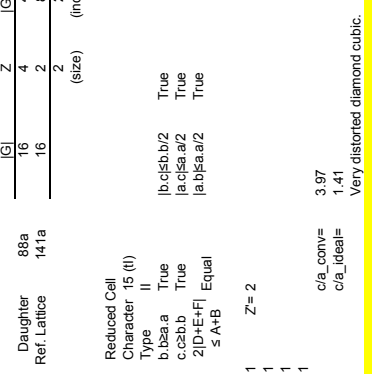

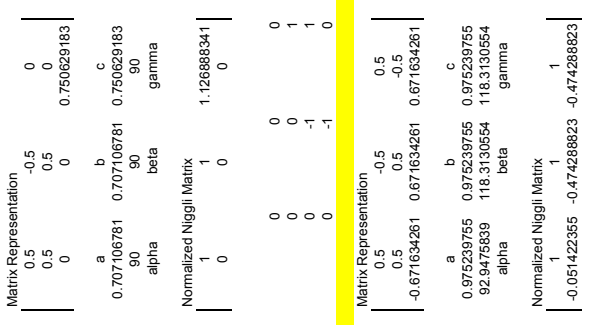

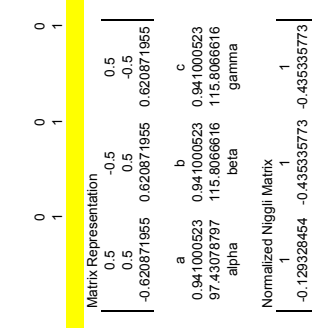

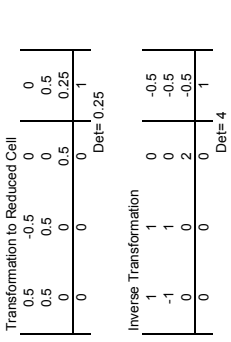
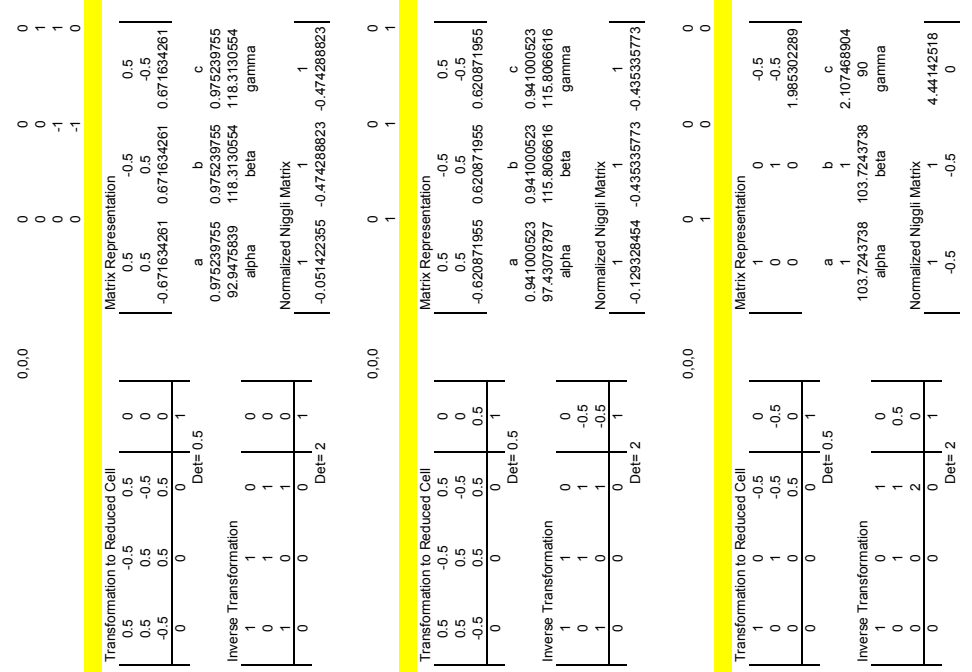

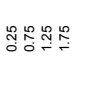

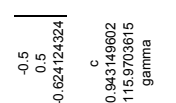

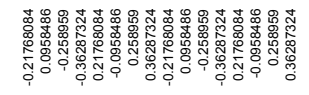

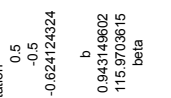

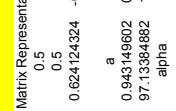

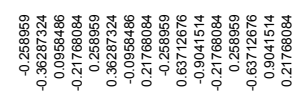

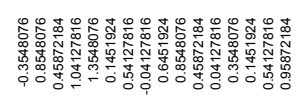
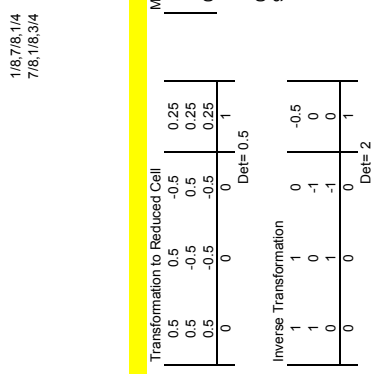
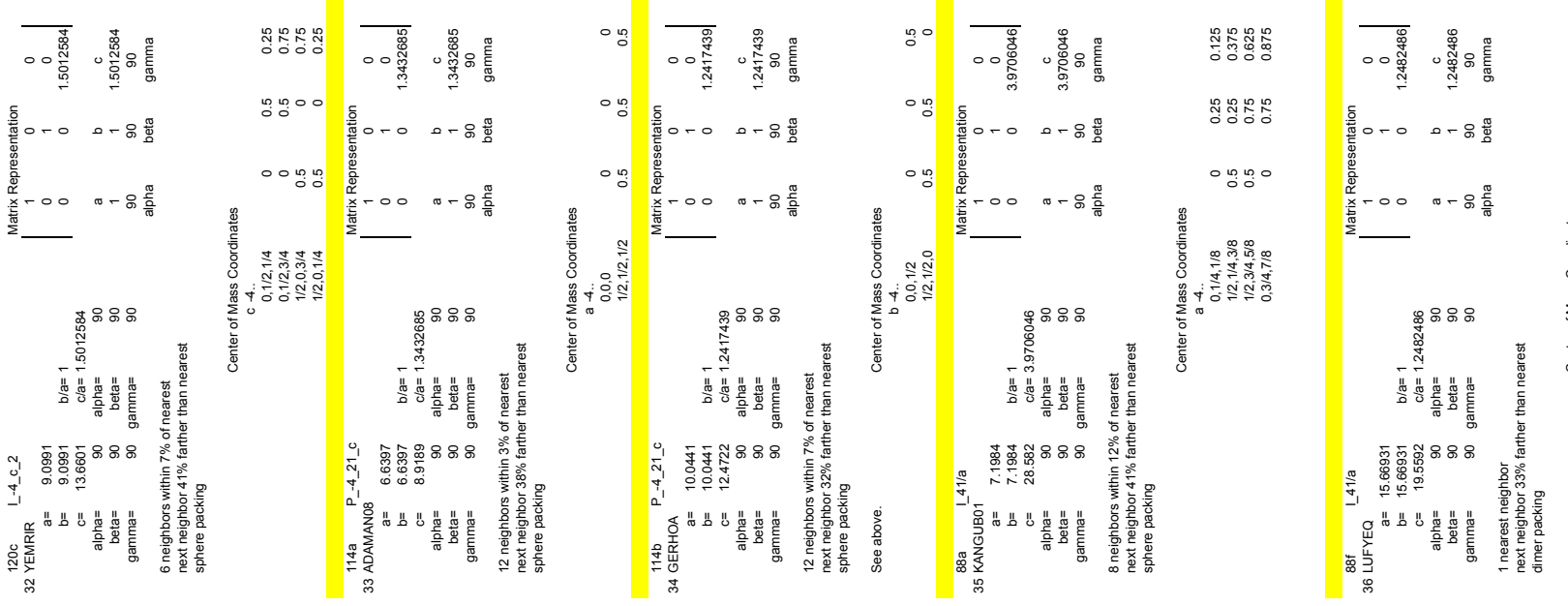

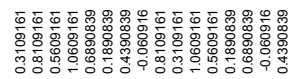

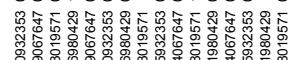

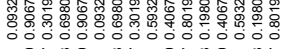

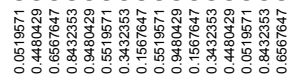
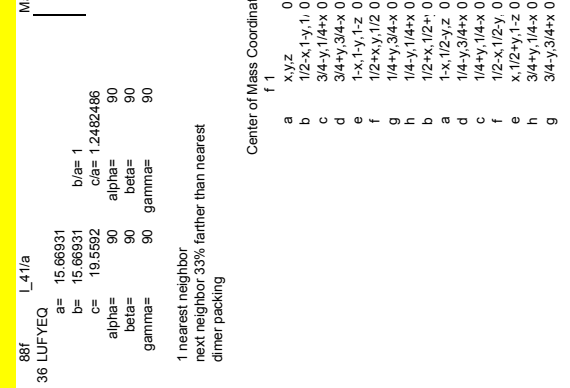

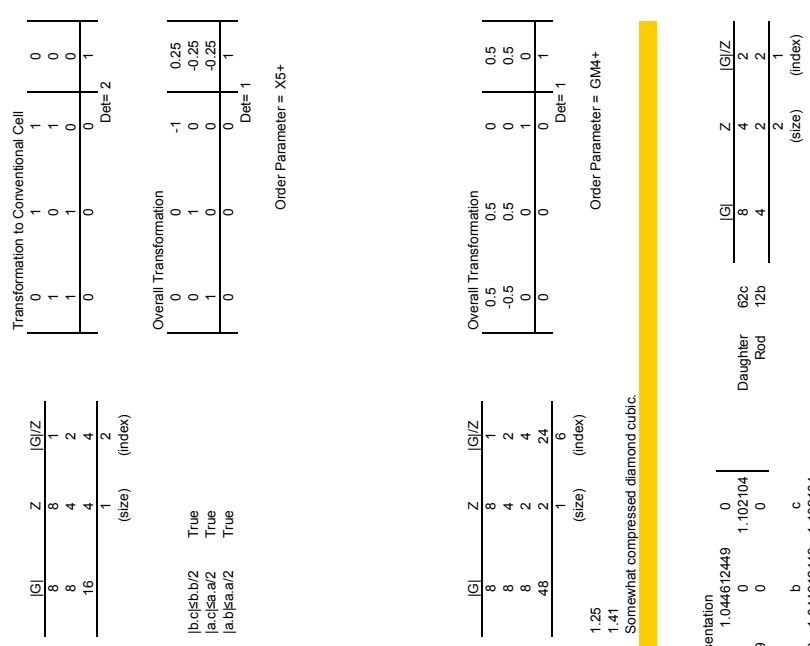

高系导

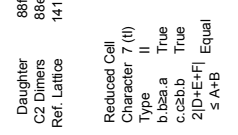

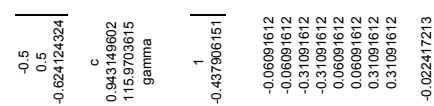

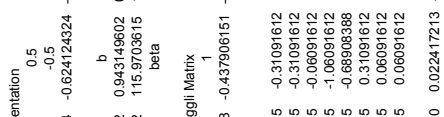

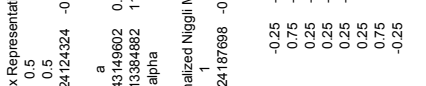

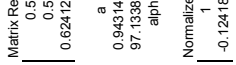

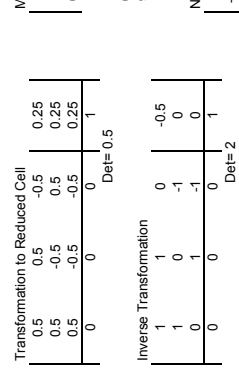

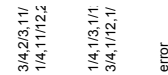

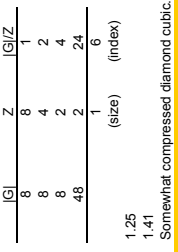

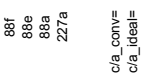

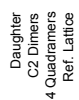

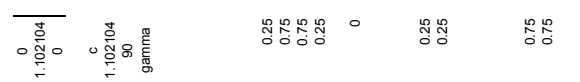

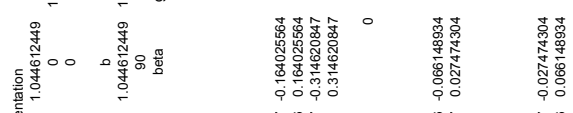

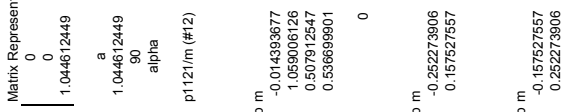

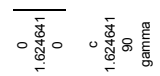

曾

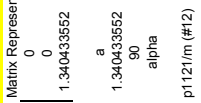

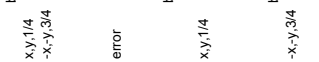
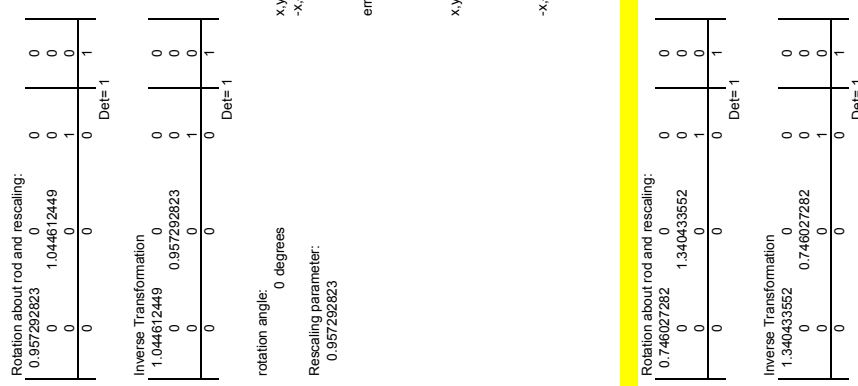

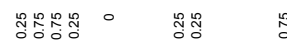
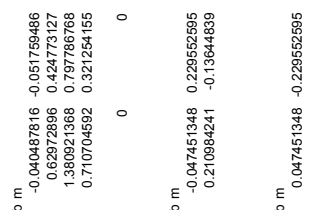

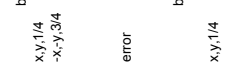

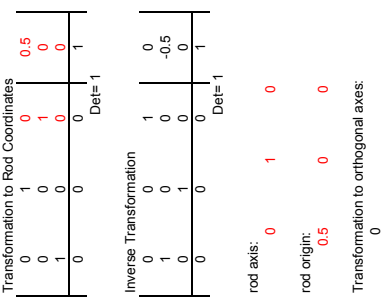

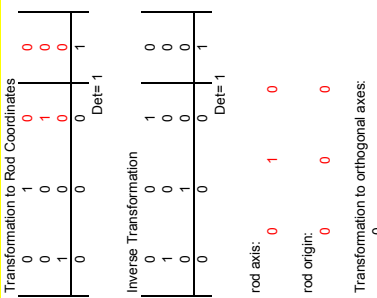
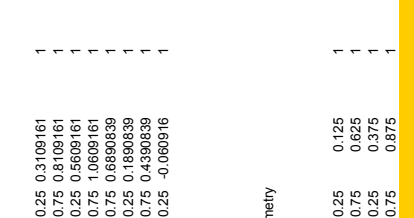

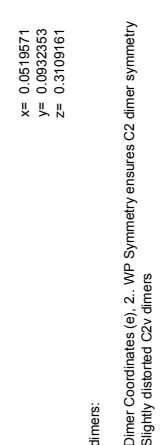
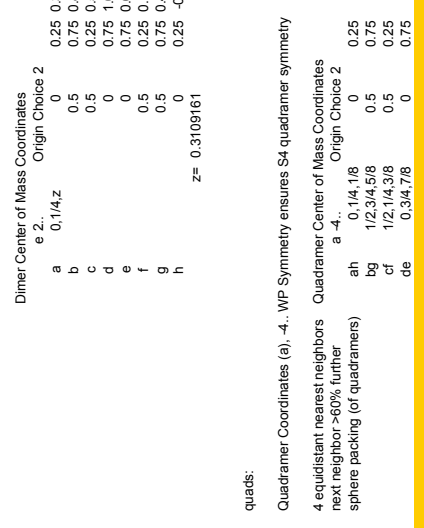

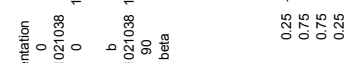

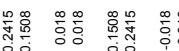

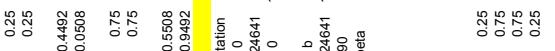

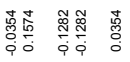

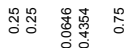

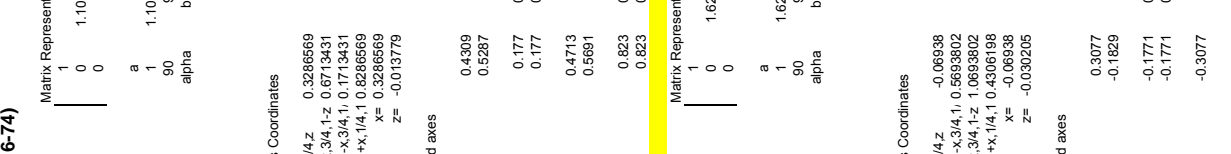

蛋

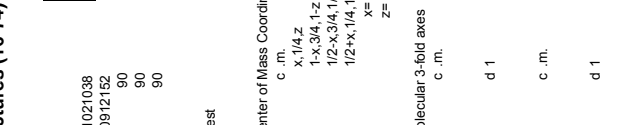

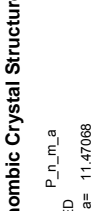

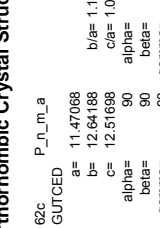

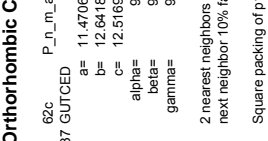

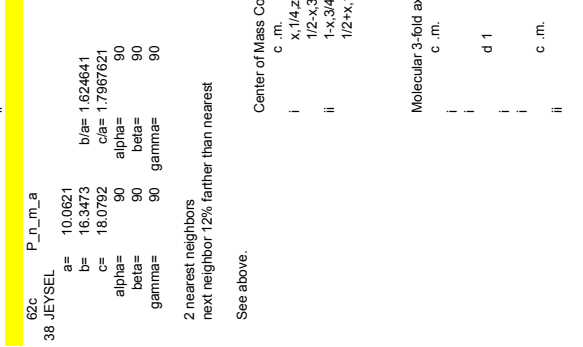




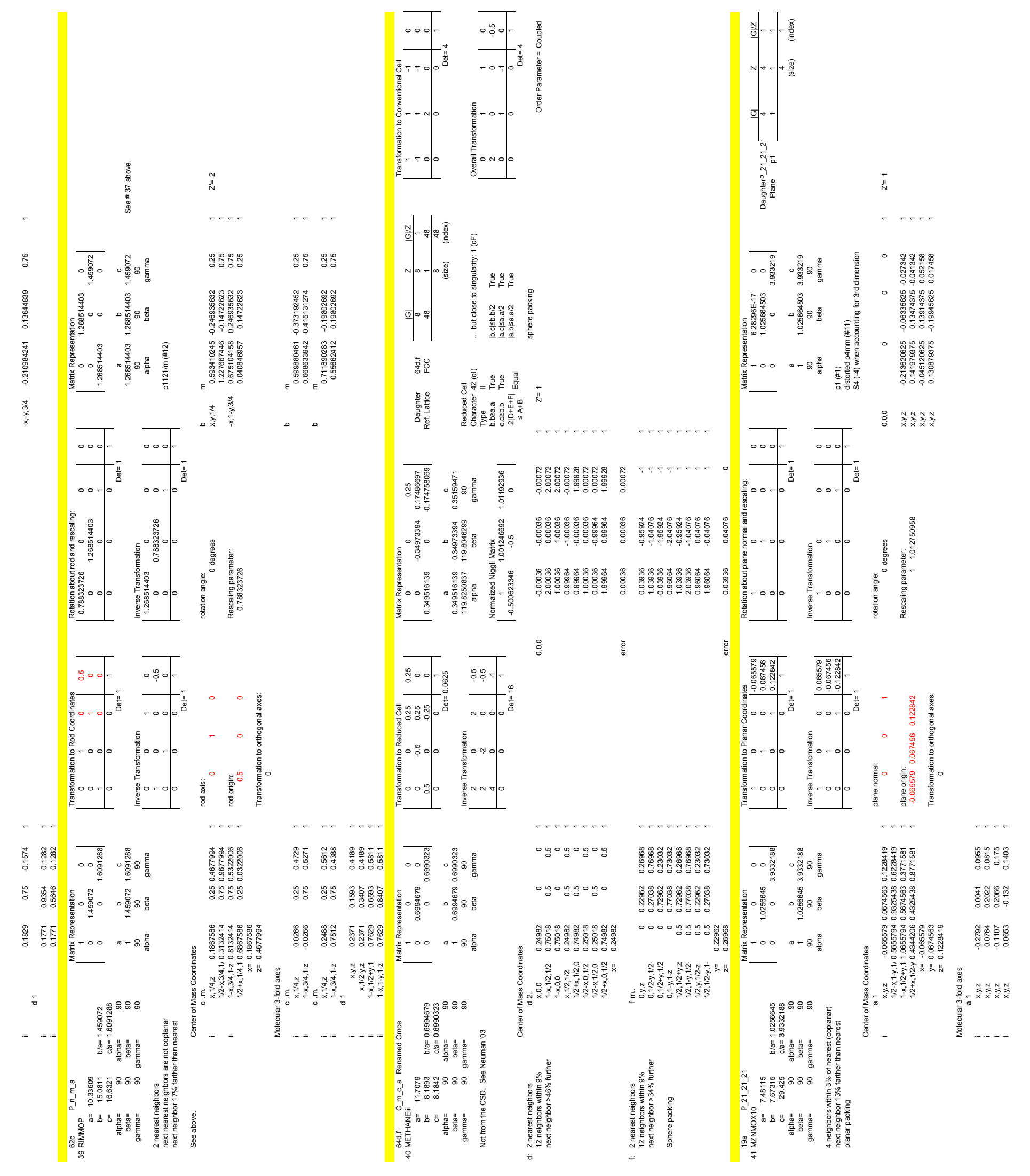




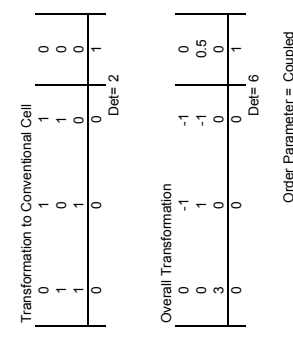

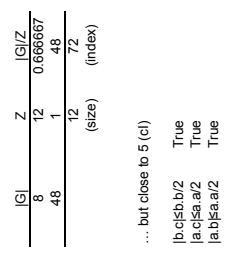

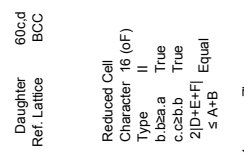
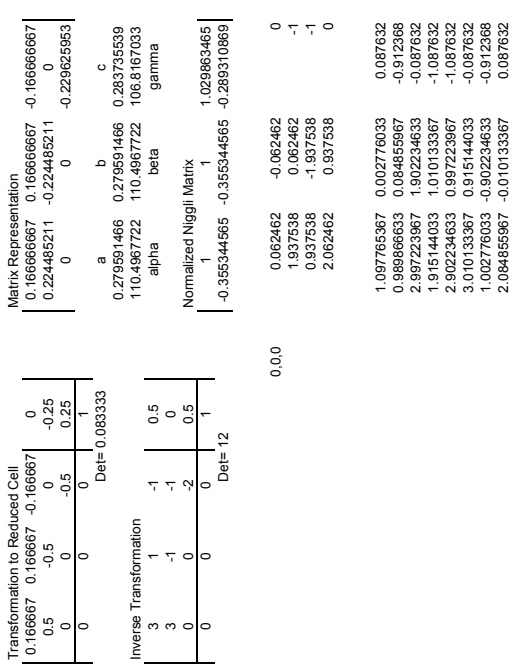

잉ำ

I.

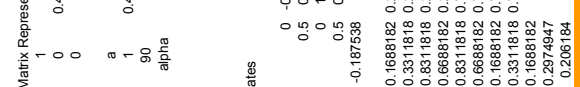

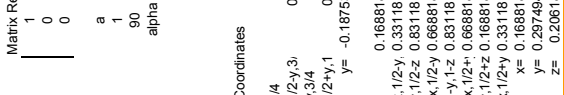

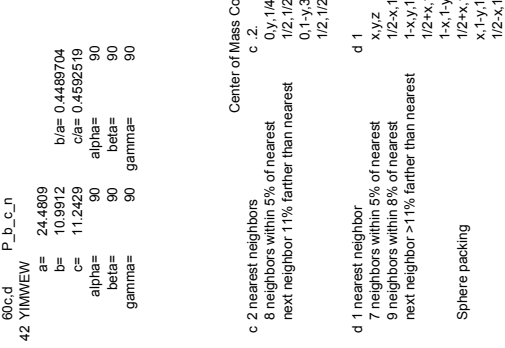

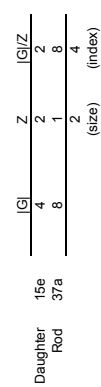

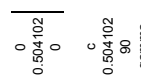

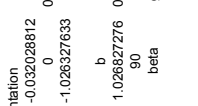

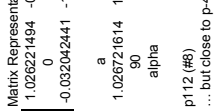

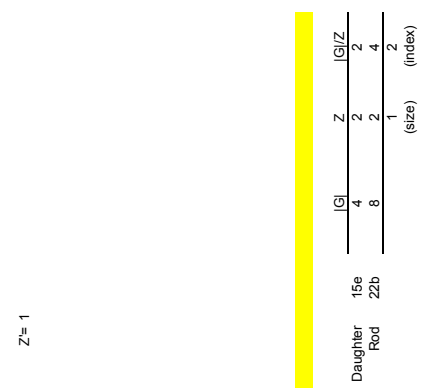

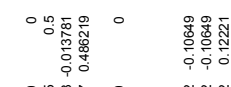

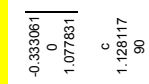

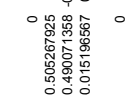

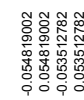

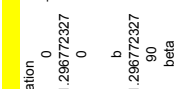

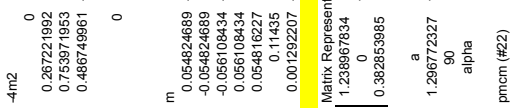

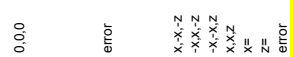

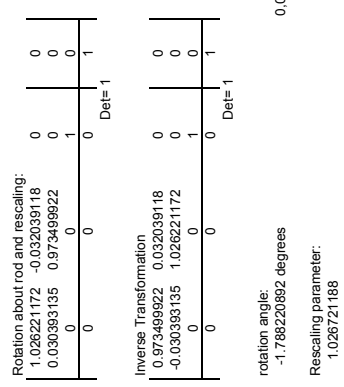

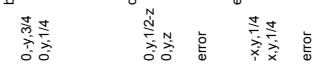
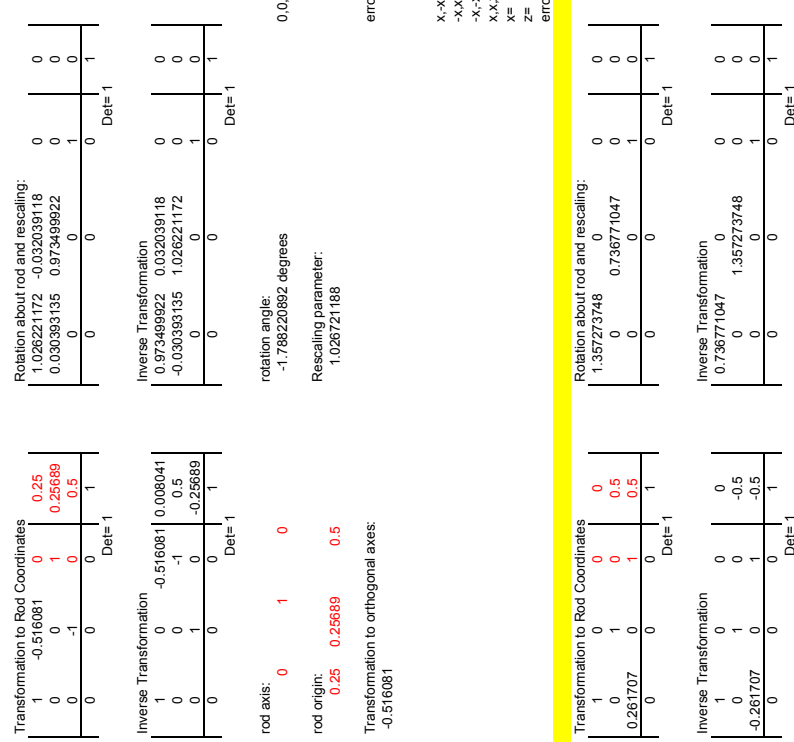

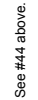

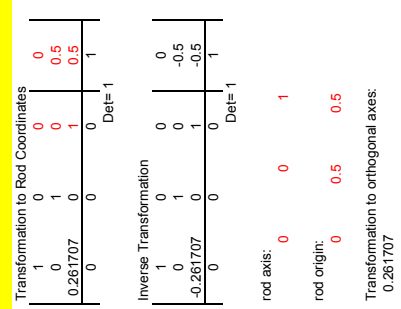

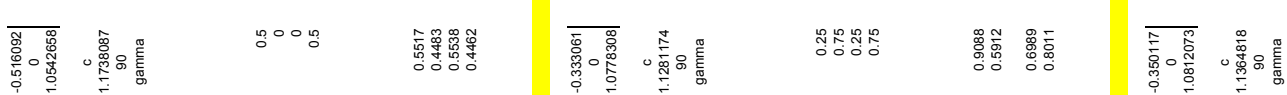

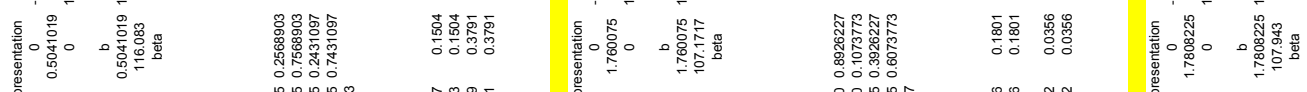

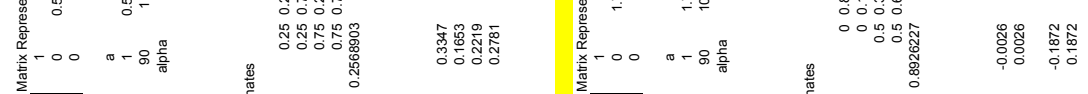
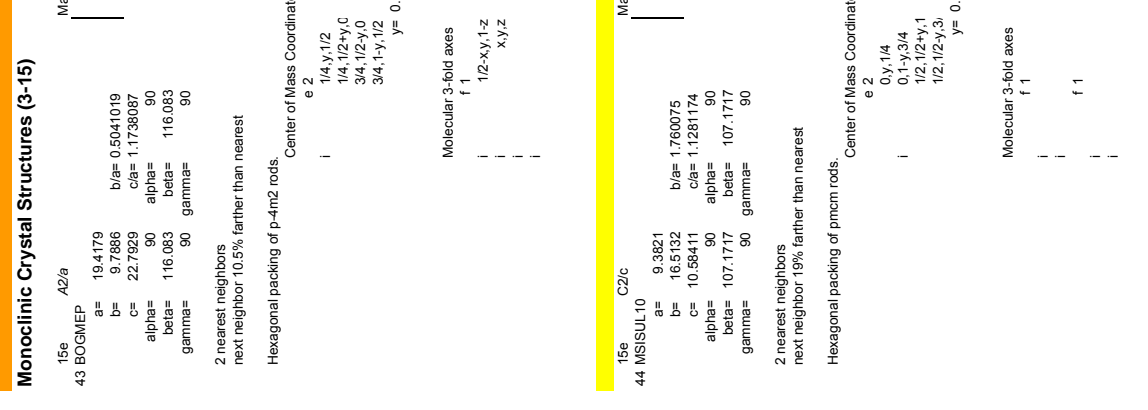

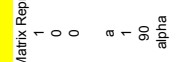
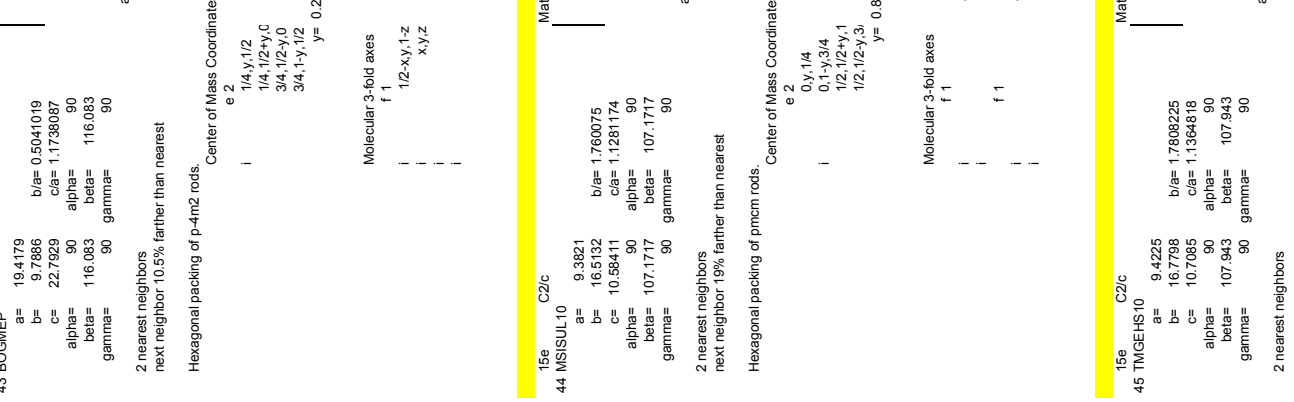


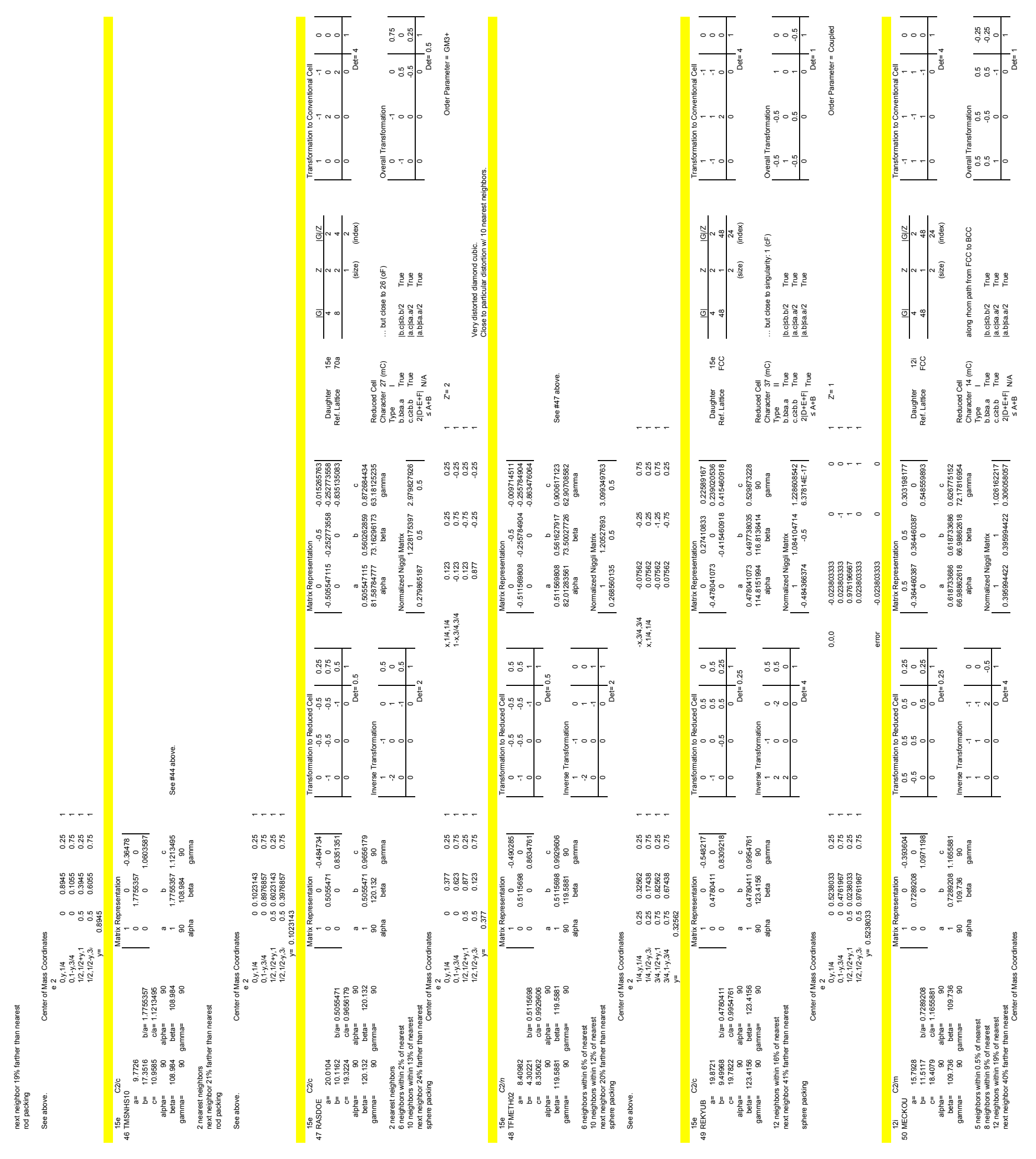




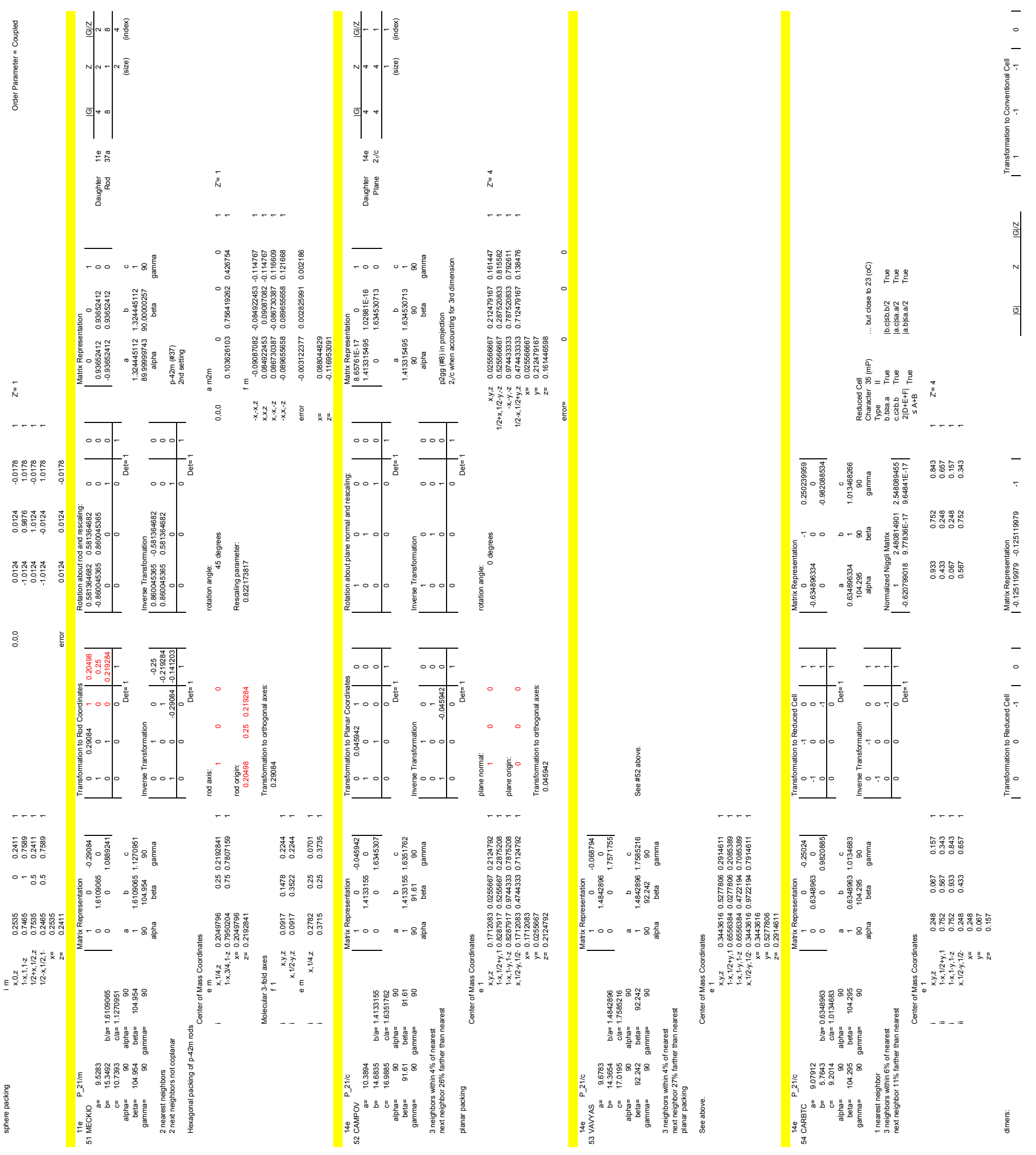




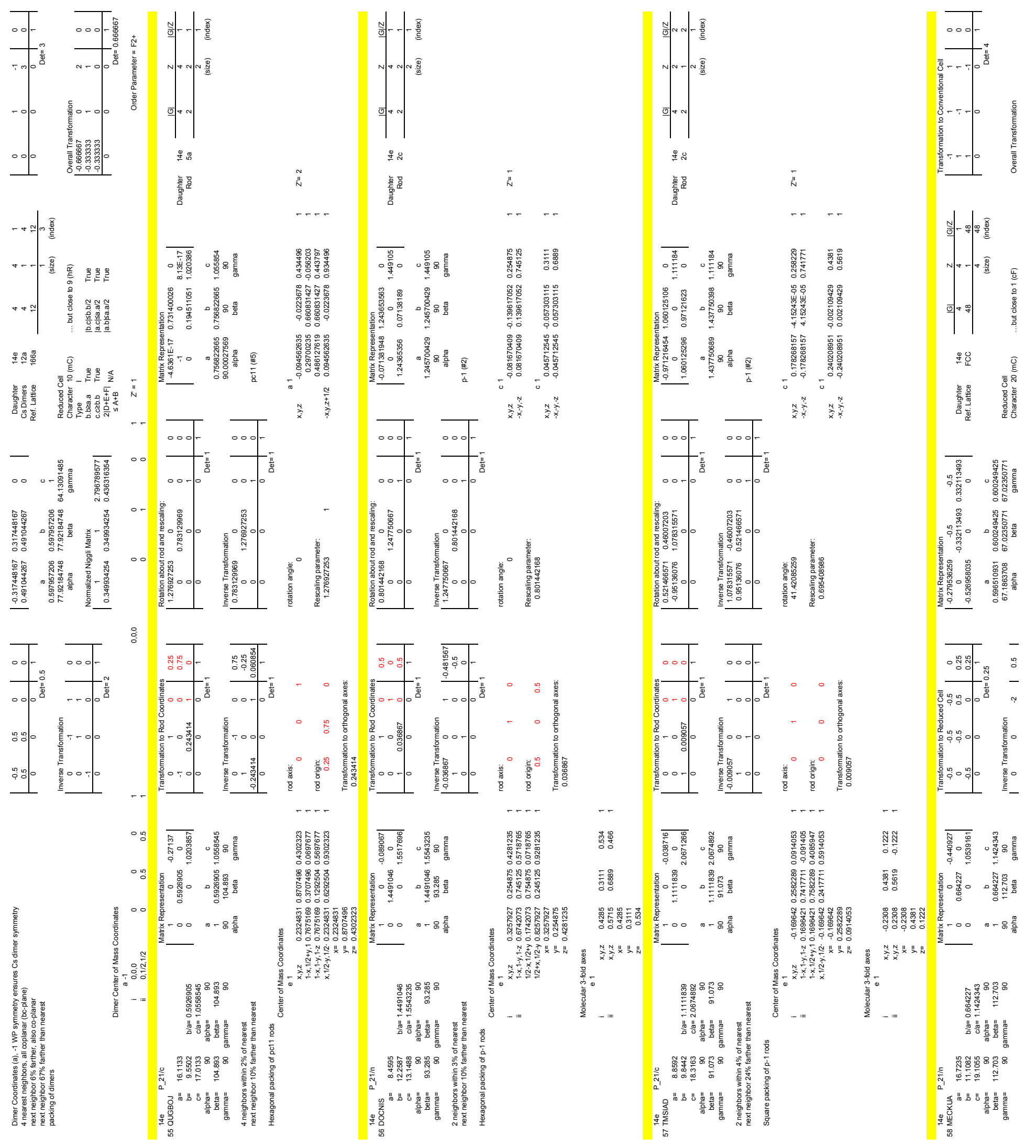



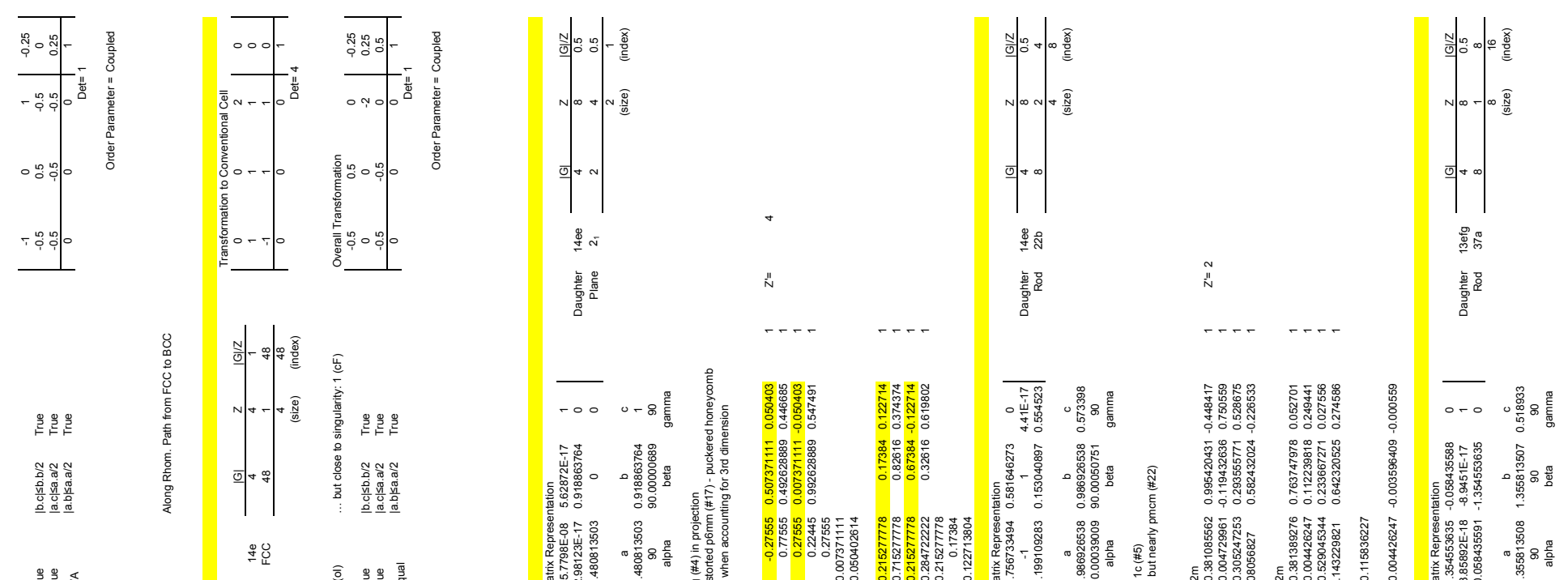

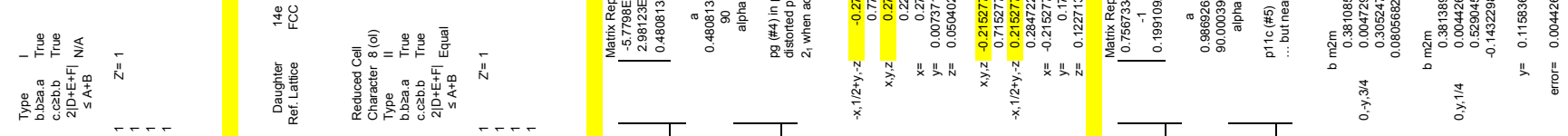
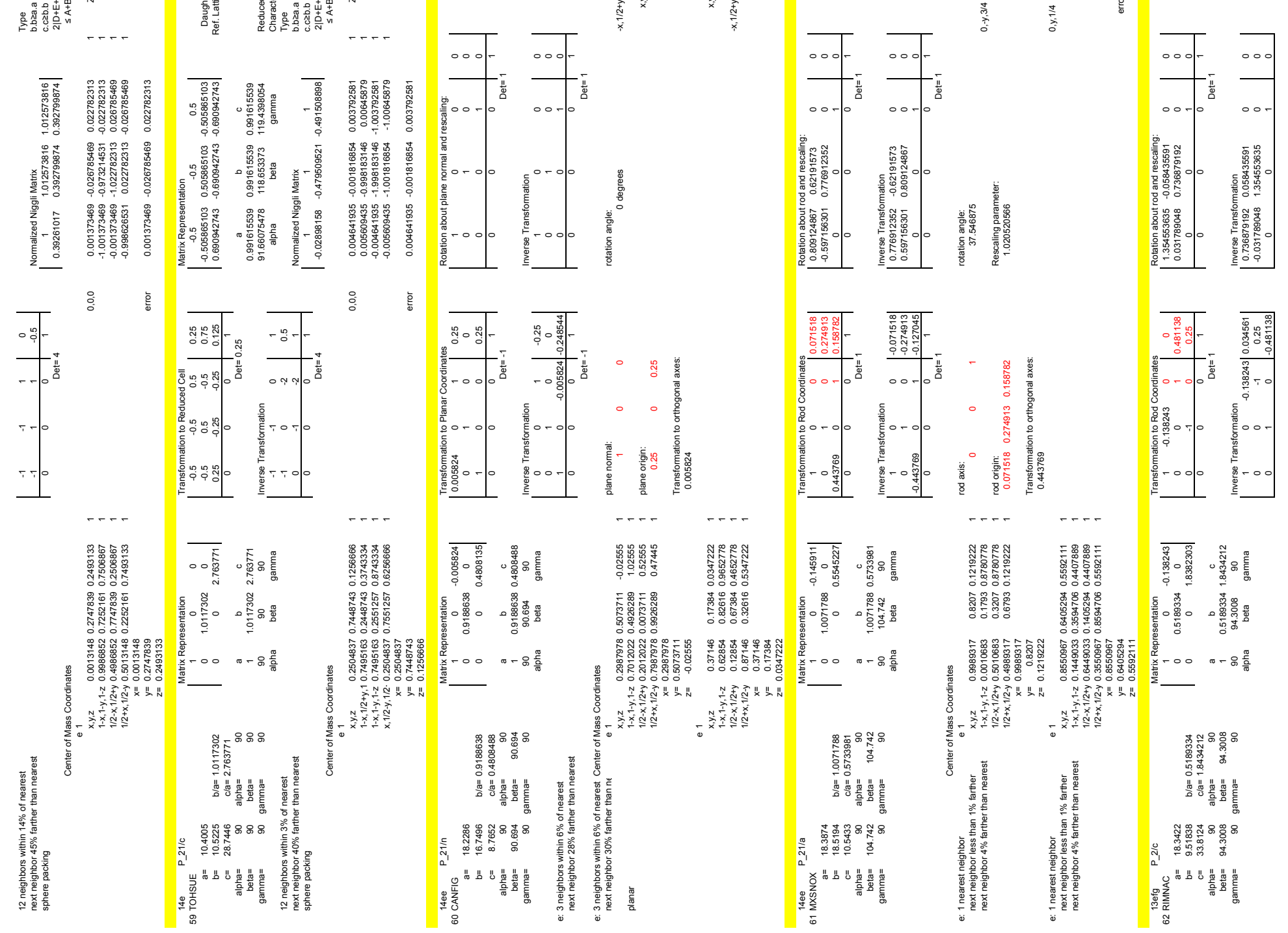

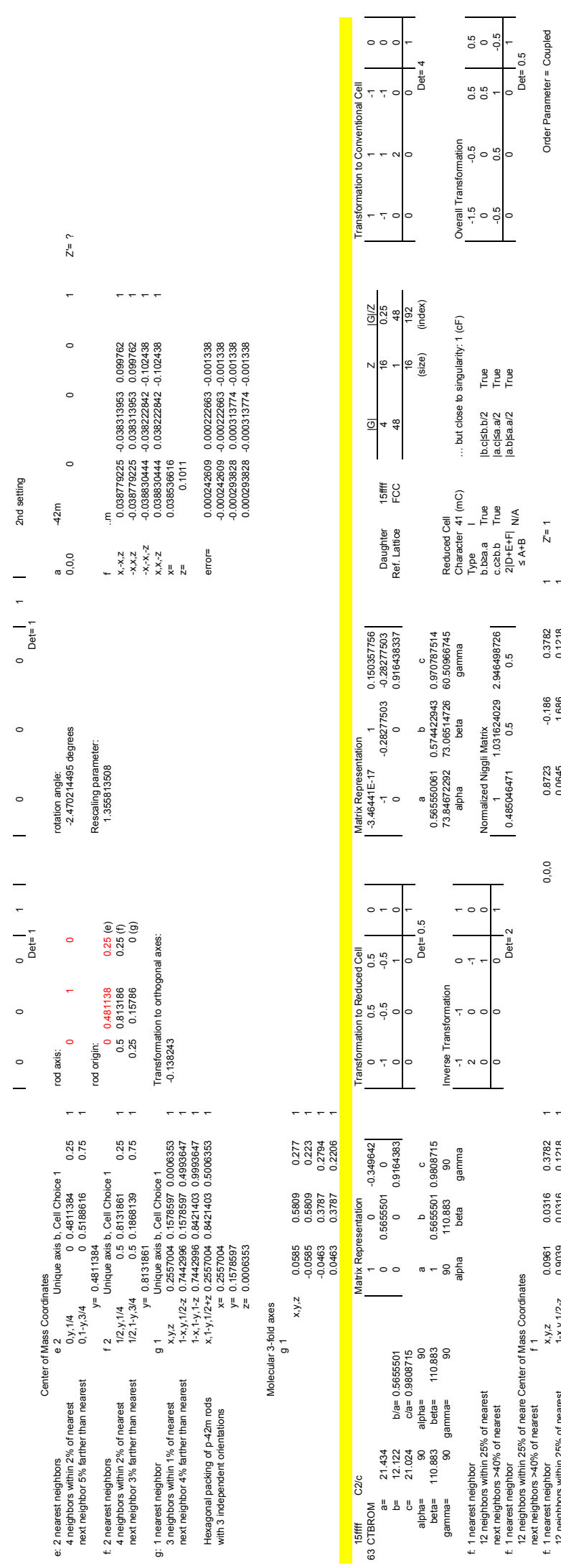

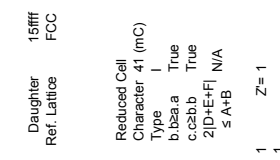
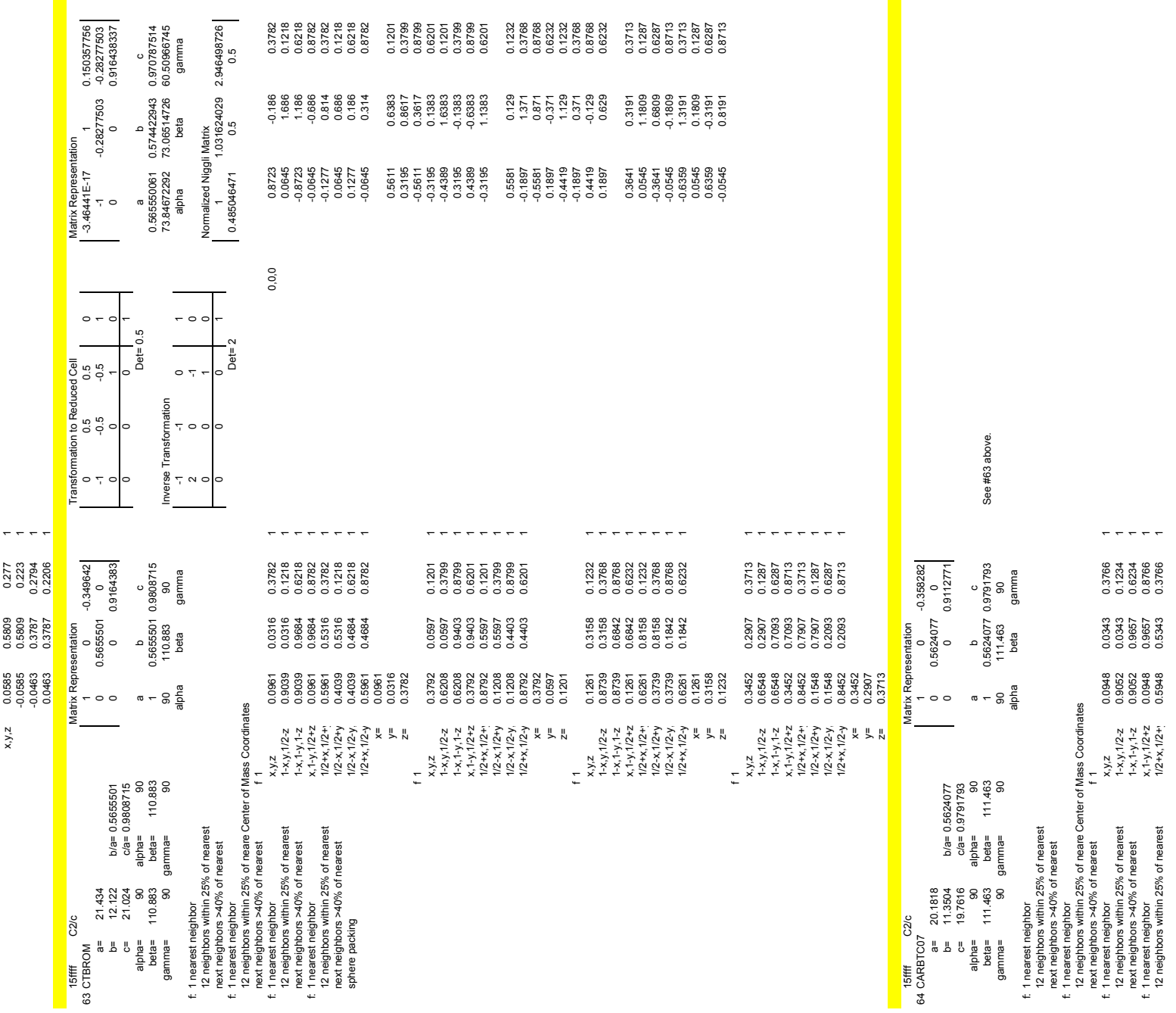


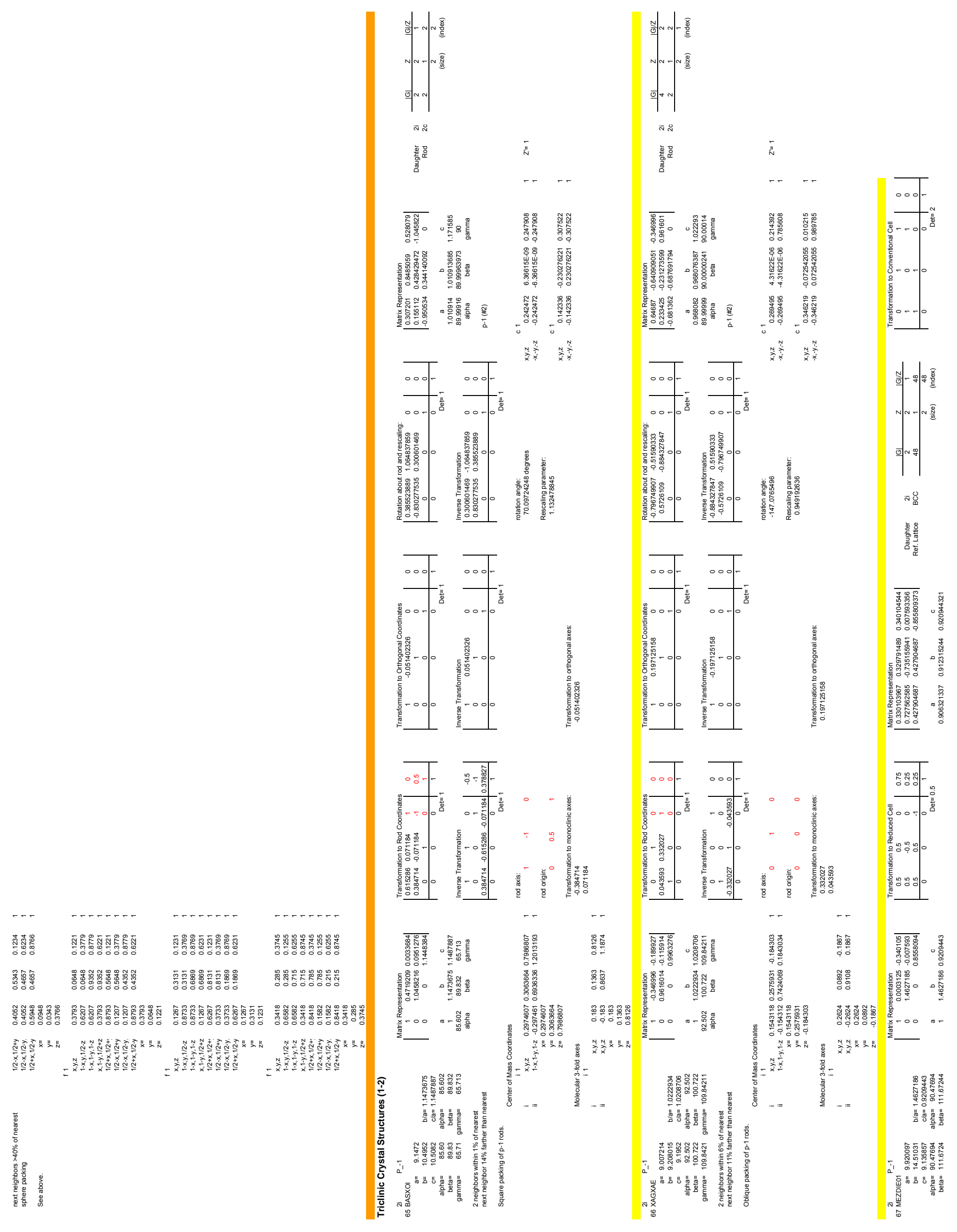




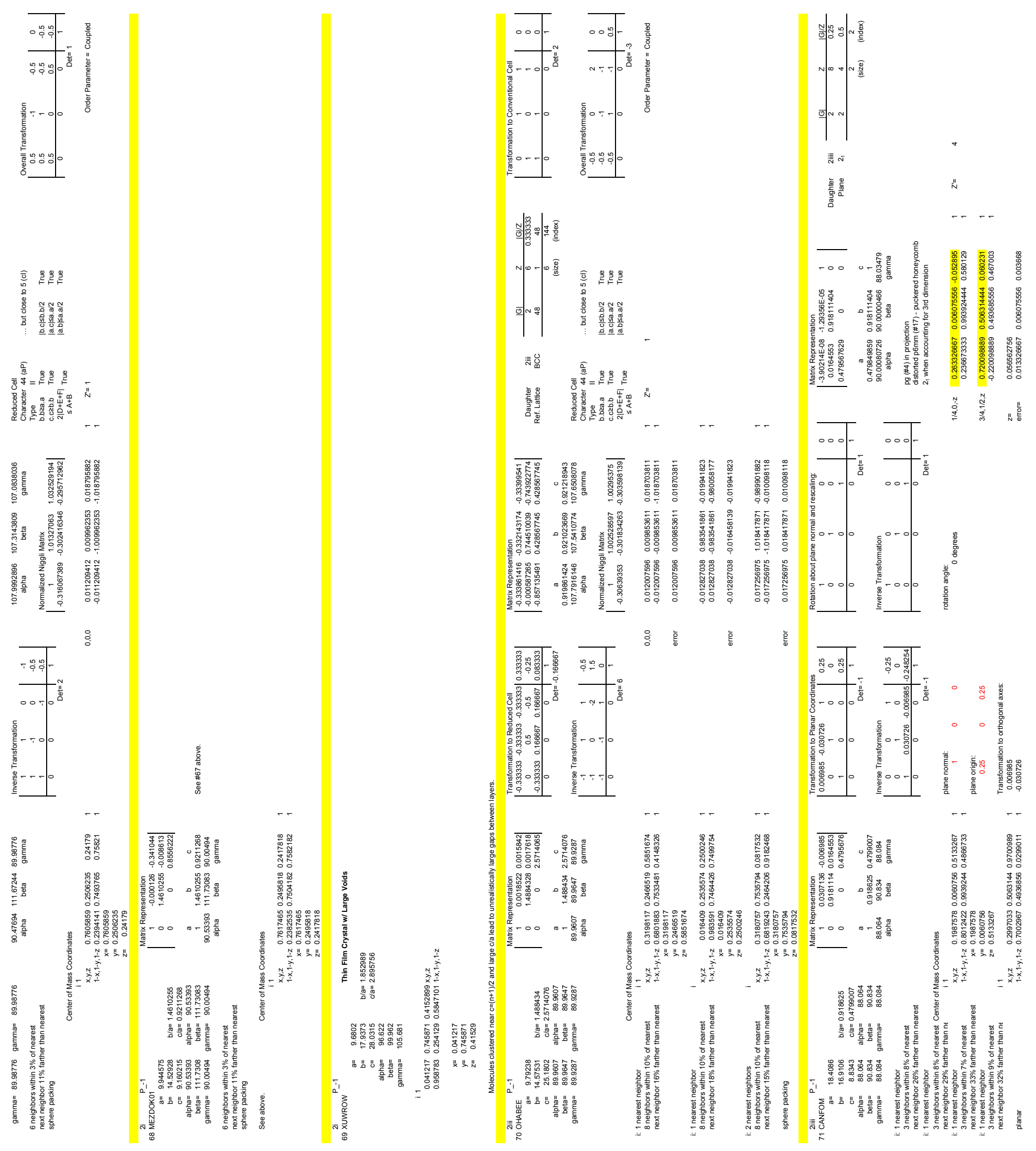




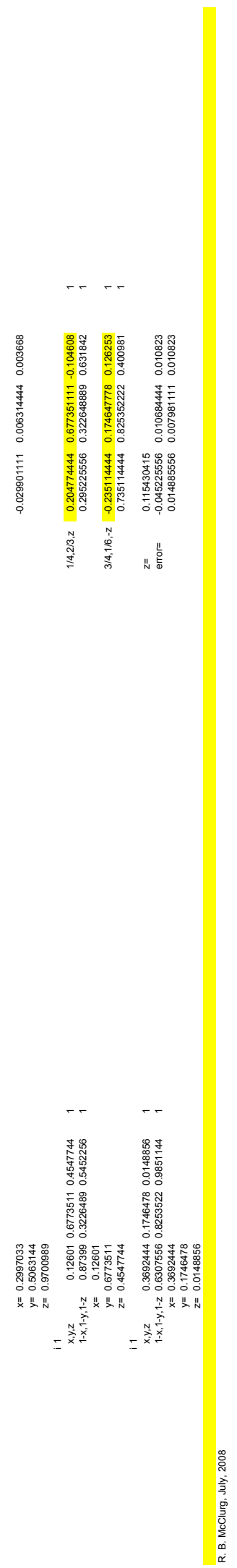



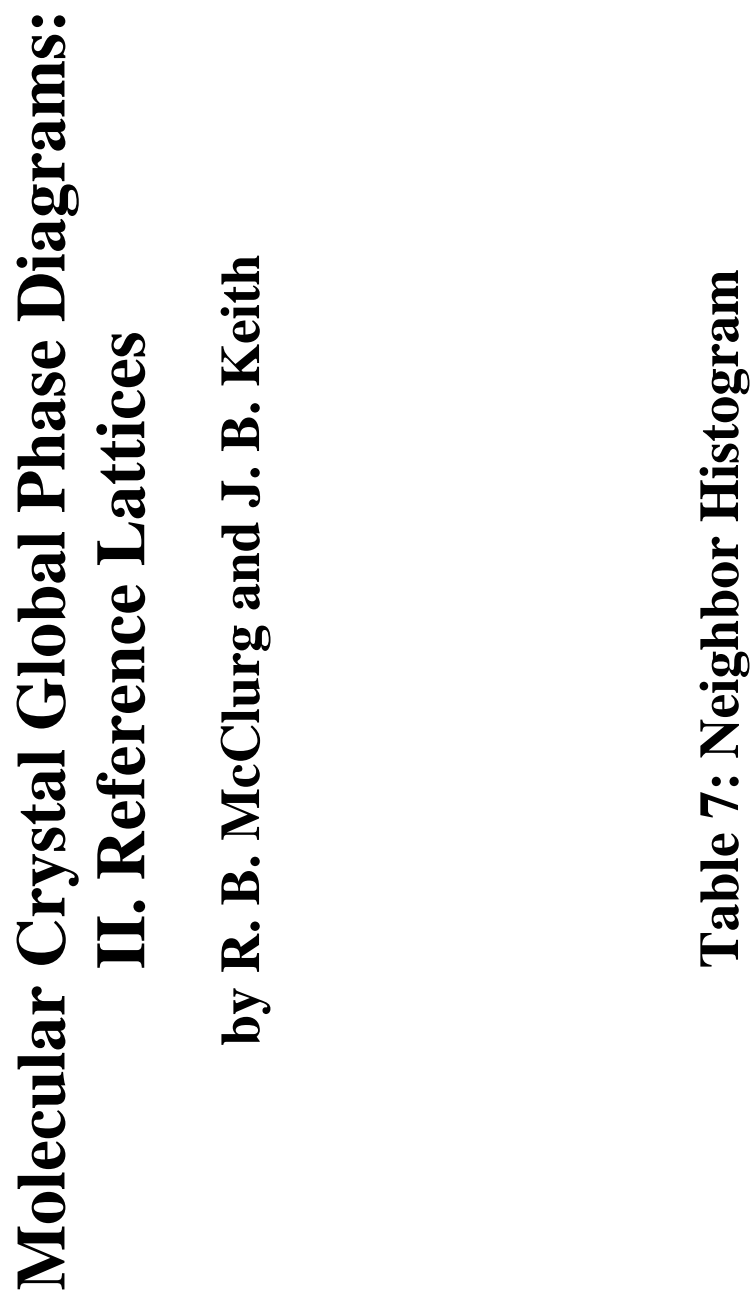


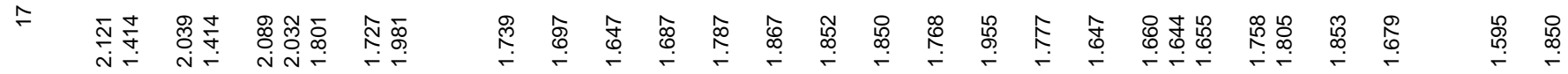

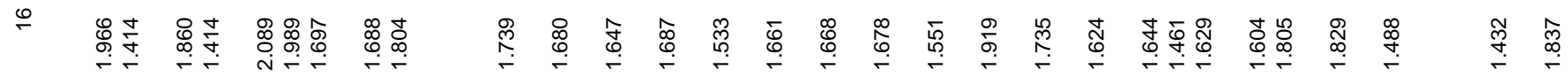

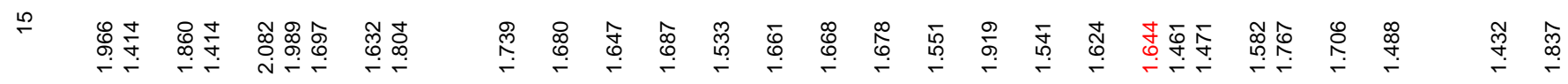

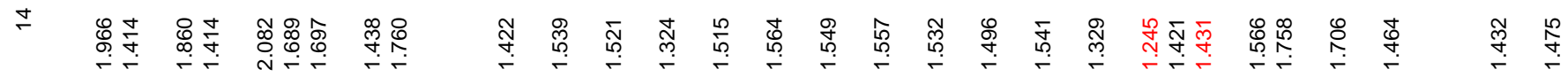

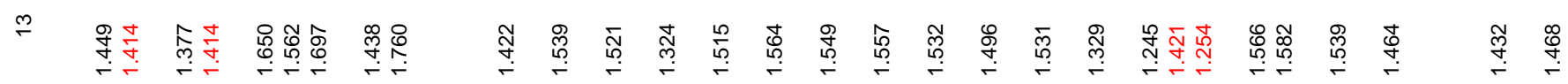

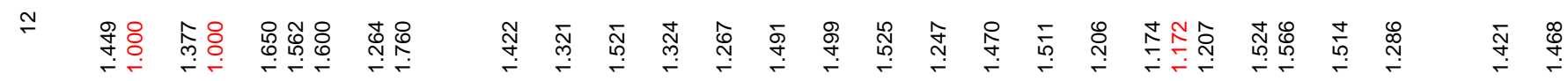

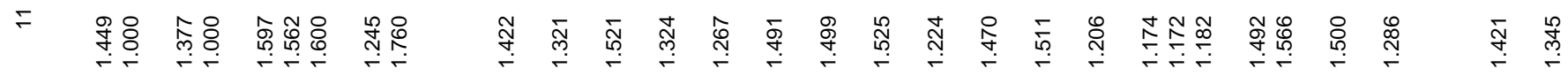

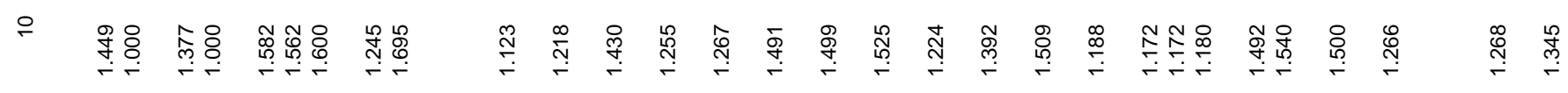

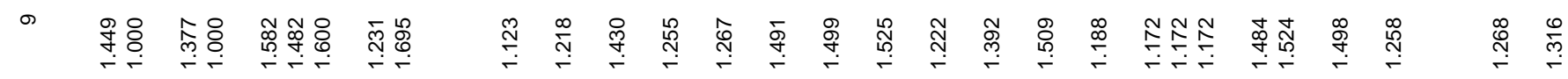

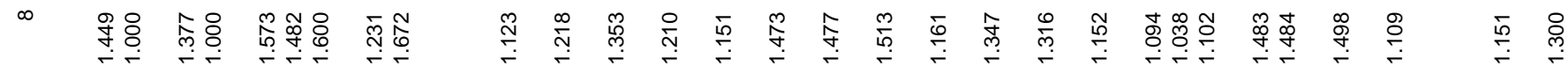

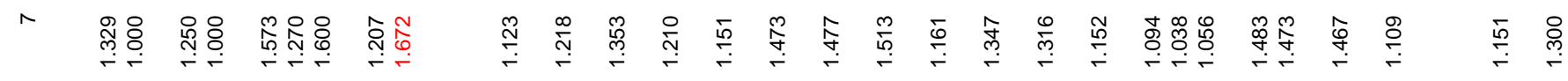

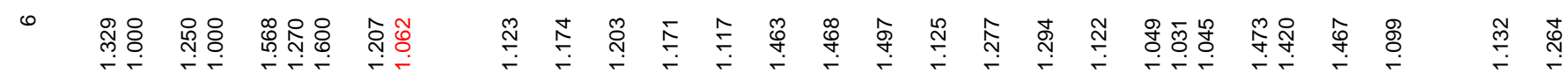

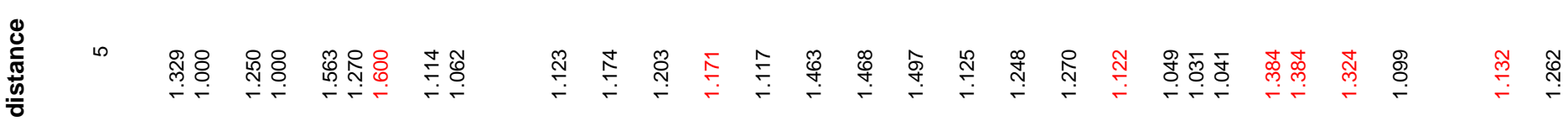

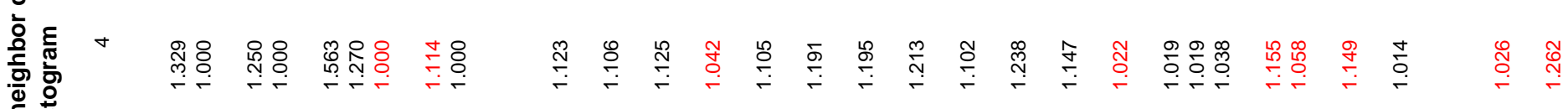

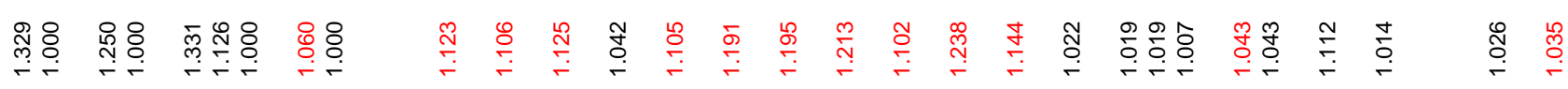

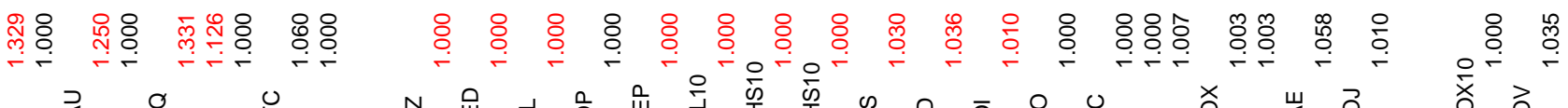

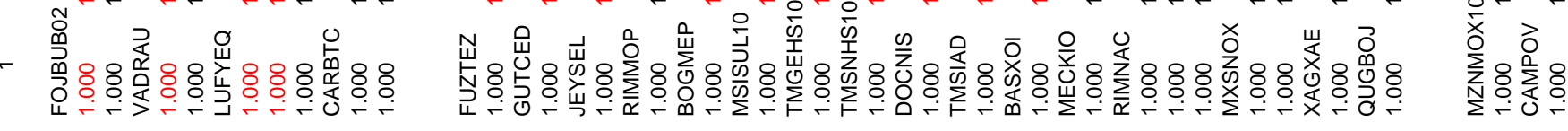

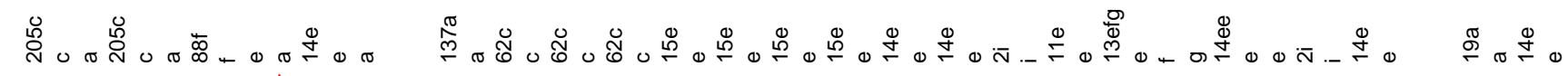

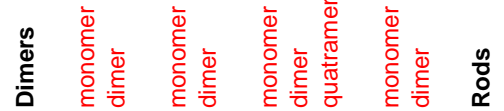




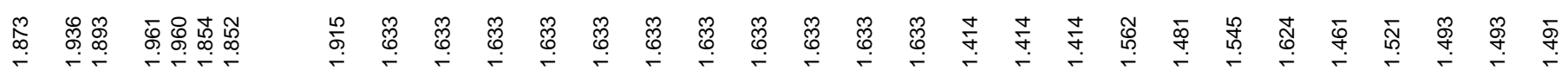

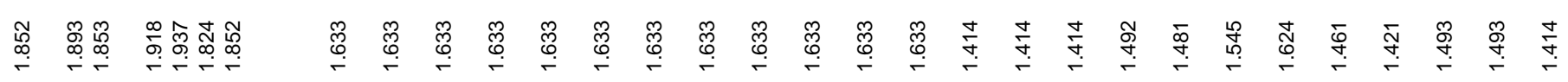

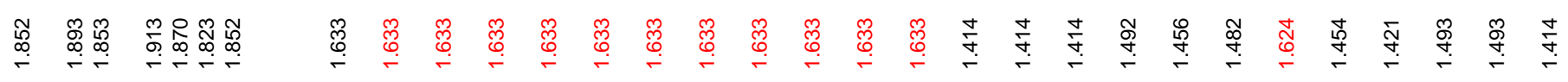

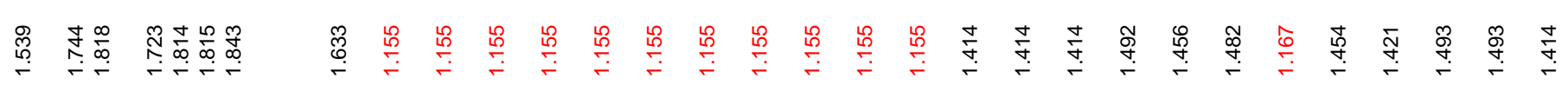

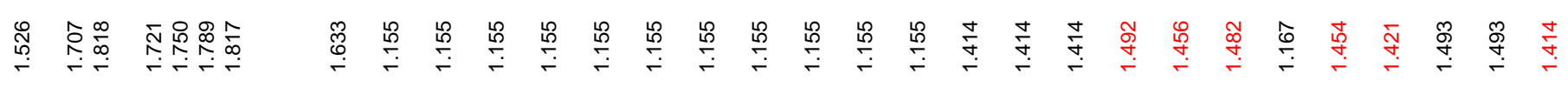

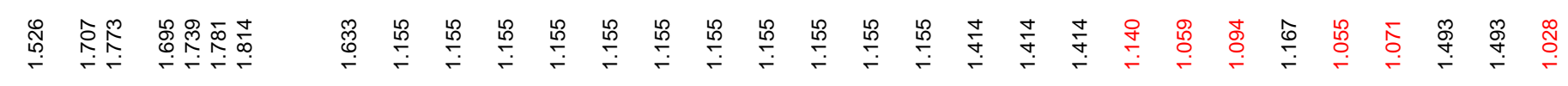

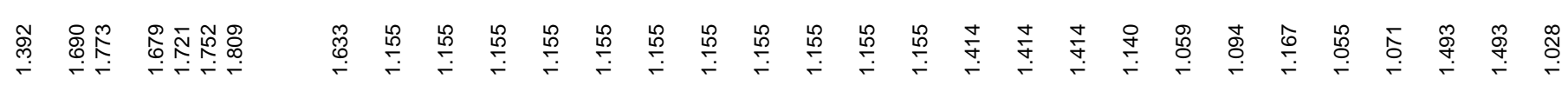

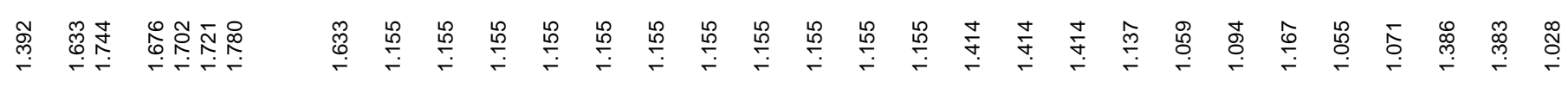

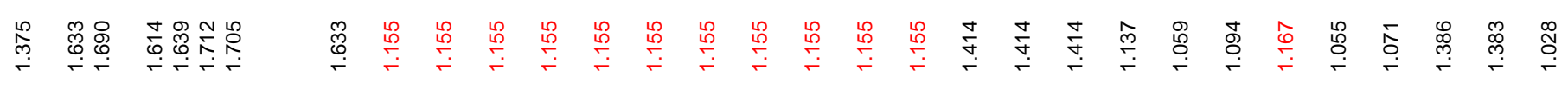

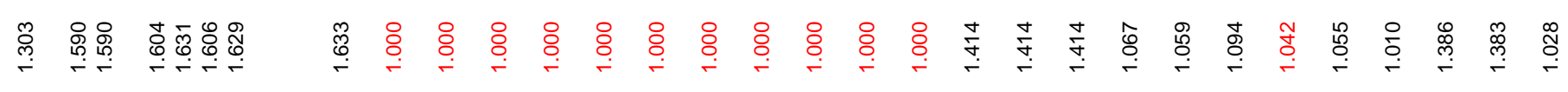

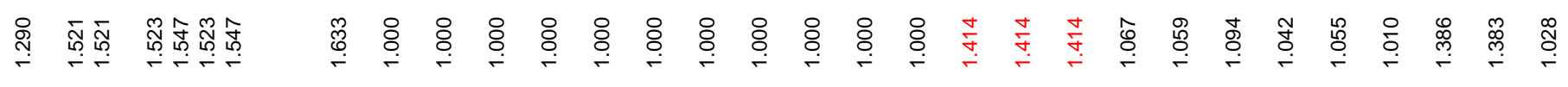

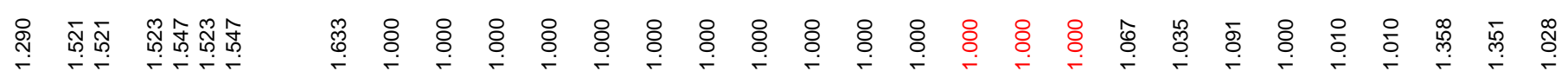

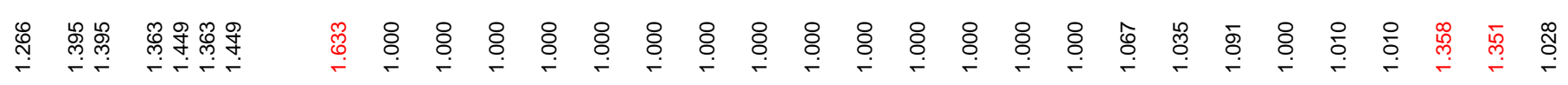

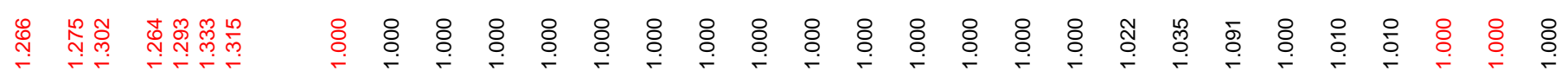

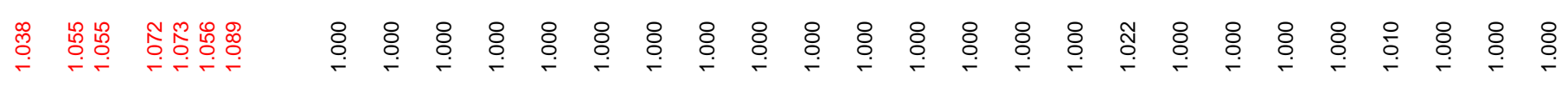

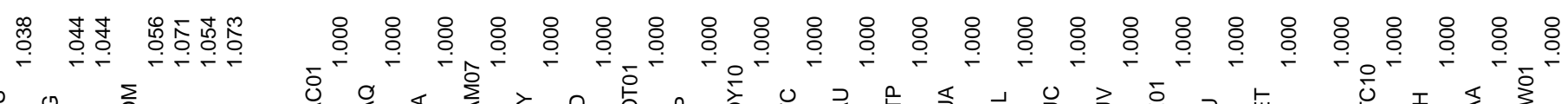

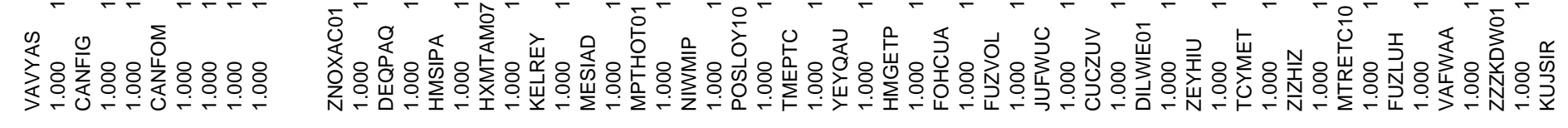

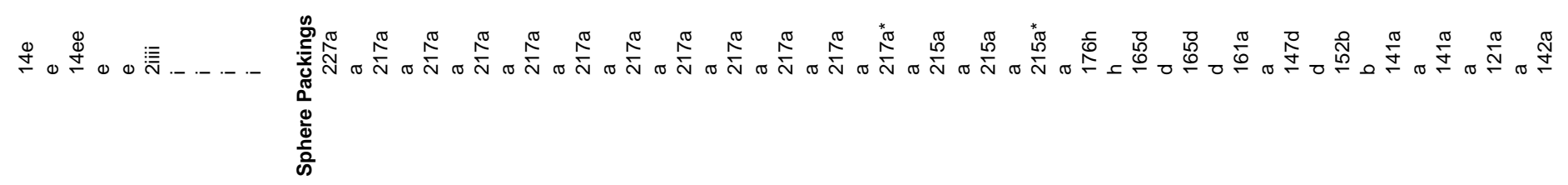




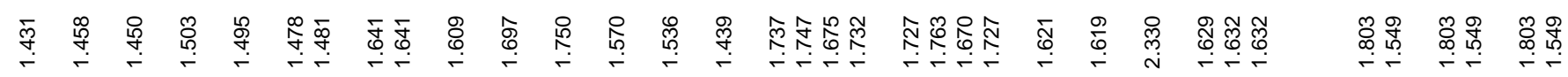

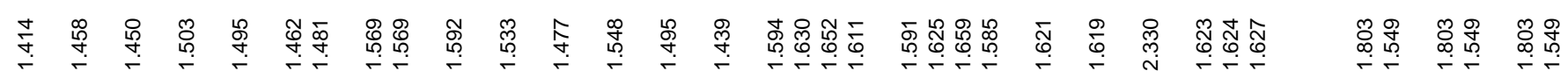

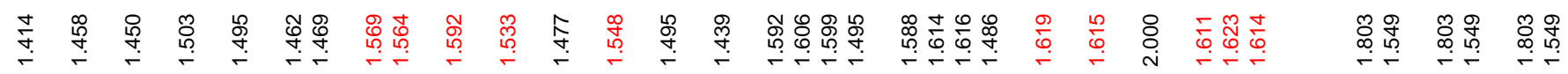

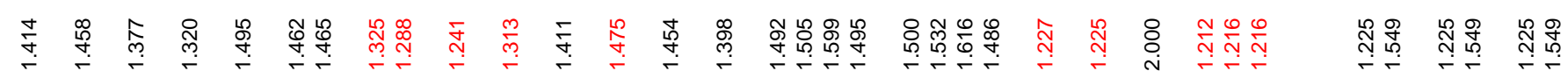

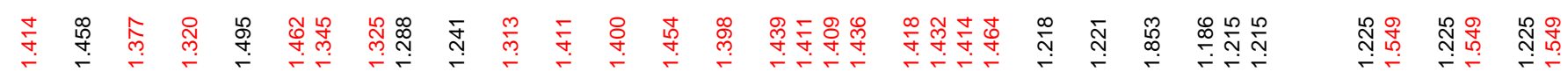

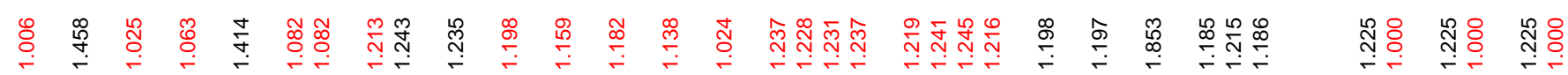

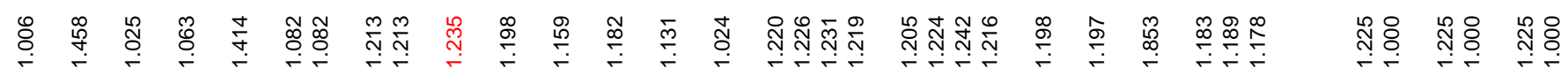

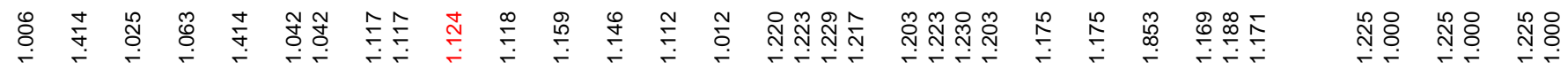

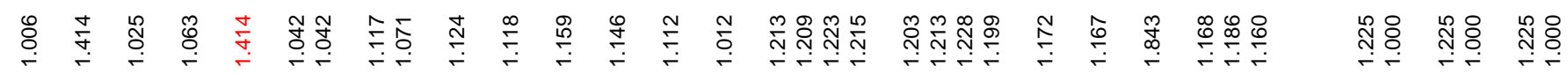

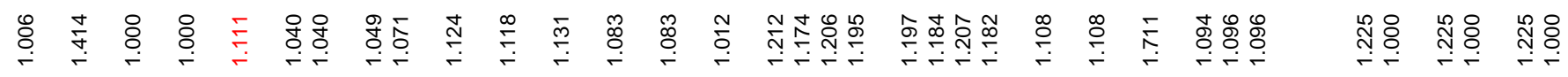

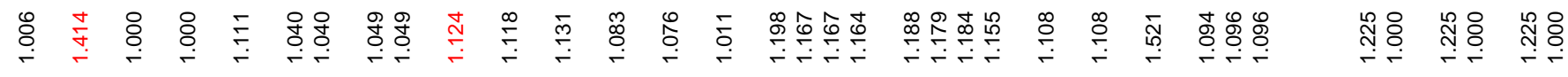

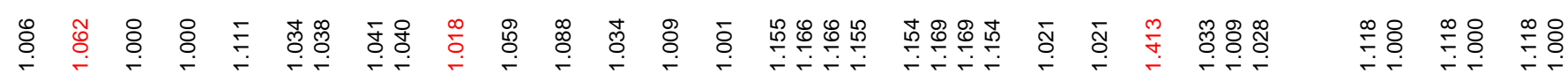

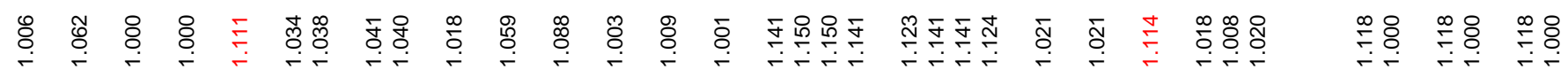

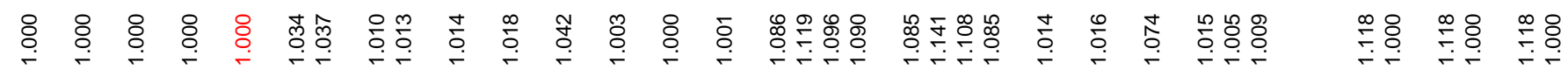

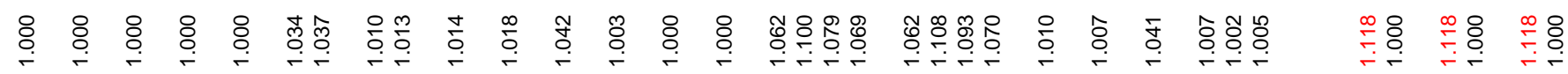

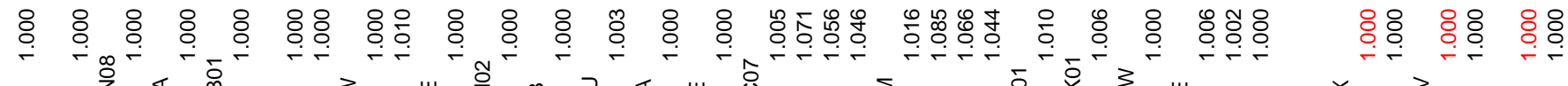

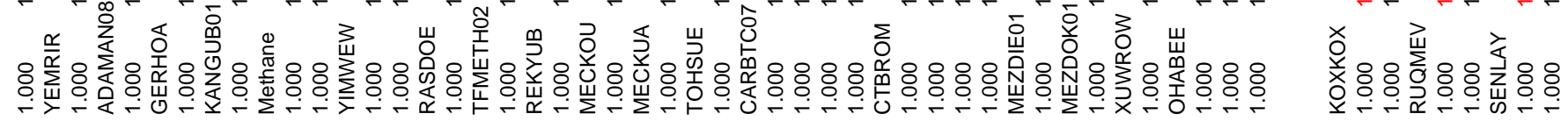

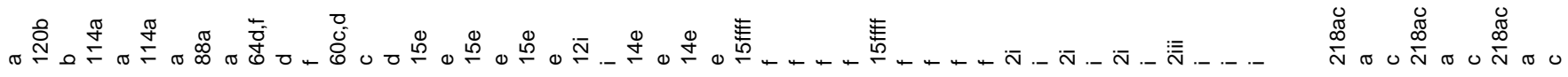




\title{
Molecular Crystal Global Phase Diagrams: II. Reference Lattices
}

\author{
by R. B. McClurg and J. B. Keith
}

Table 8: Order Parameters 
Order Parameters Calculated Using:

COPL as found on:

H.T.Stokes and D.M.Hatch, (2002). ISOTROPY, stokes.byu.edu/isotropy.html.

\section{Sphere Packings:}

ZNOXAC01

Parent: 227 oh-7, Fd-3m, F4_1/d-32/m, origin choice 2

Subgroup: 227 Oh-7, Fd-3m, F4_1/d-32/m, origin choice 2

Lattice vectors:

100

$\odot 10$

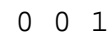

origin: $\odot \odot \odot$

Irrep Dir Subgroup Size

GM1+ (a) $227 \mathrm{Fd}-3 \mathrm{~m} \quad 1$

GM1+ is the primary OP.

DEQPAQ, et al.

Parent: 229 Oh-9, Im-3m, I4/m-32/m

Subgroup: $217 \mathrm{Td}-3$, I-43m, I-43m

Lattice vectors:

100

$\begin{array}{lll}0 & 1 & 0\end{array}$

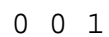

origin: $\odot \odot \odot$

Irrep Dir Subgroup Size

GM1+ (a) 229 Im-3m 1

GM2 - (a) 217 I-43m 1

GM2 - is the primary OP.

FOHCUA, et al.

Parent: 221 oh-1, Pm-3m, P4/m-32/m

Subgroup: $215 \mathrm{Td}-1, \mathrm{P}-43 \mathrm{~m}, \mathrm{P}-43 \mathrm{~m}$

Lattice vectors:

$1 \odot \odot$

$\begin{array}{lll}0 & 1 & 0\end{array}$

$\odot \odot 1$

origin: $\odot \odot \odot$

Irrep Dir Subgroup Size

GM1+ (a) $221 \mathrm{Pm}-3 \mathrm{~m} \quad 1$

GM2 - (a) 215 P-43m 1

GM2 - is the primary OP.

CUCZUV

Parent: 194 D6h-4,P6_3/mmc, P6_3/m2/m2/C

Subgroup: $176 \mathrm{C} 6 \mathrm{~h}-2, \mathrm{P} 6 \_3 / \mathrm{m}, \mathrm{P} 6 \_3 / \mathrm{m}$

Lattice vectors:

$1-10$

120

$\odot \begin{array}{ccc}0 & 0 & 1\end{array}$

origin: $\odot \odot 1 / 2$

$\begin{array}{lllr}\text { Irrep } & \text { Dir } & \text { Subgroup } & \text { Size } \\ \text { GM1+ } & (\mathrm{a}) & 194 \mathrm{P} 6 \_3 / \mathrm{mmc} & 1 \\ \text { GM2+ } & (\mathrm{a}) & 176 \mathrm{P} 6 \_3 / \mathrm{m} & 1 \\ \text { K1 } & (\mathrm{a}, 0) & 193 \mathrm{P} 6 \text { 3/mcm } & 3 \\ \text { K4 } & (\mathrm{a}, 0) & 176 \mathrm{P} 6 \text { 3/m } & 3\end{array}$

$\mathrm{K} 4$ is the primary OP. 
DILWIE01 \& ZEYHIU

Parent: 194 D6h-4, P6_3/mmc, P6_3/m2/m2/C

Subgroup: $165 \mathrm{D} 3 \mathrm{~d}-4, \mathrm{P}-3 \mathrm{c} 1, \mathrm{P}-32 / \mathrm{C} 1$

Lattice vectors:

100

$\begin{array}{lll}0 & 1 & 0\end{array}$

$\odot \odot 2$

origin: $\odot \odot \odot$

$\begin{array}{lllc}\text { Irrep } & \text { Dir } & \text { Subgroup } & \text { Size } \\ \text { GM1+ } & \text { (a) } & \text { 194 P6_3/mmc } & 1 \\ \text { GM3+ } & \text { (a) } & 164 \text { P-3m1 } & 1 \\ \text { A2 } & (\mathrm{a}, \text { a }) & 165 \text { P-3c1 } & 2\end{array}$

A2 is the primary OP.

\section{TCYMET}

Parent: 229 oh-9, Im-3m, I4/m-32/m

Subgroup: $161 \mathrm{C} 3 \mathrm{~V}-6, \mathrm{R} 3 \mathrm{C}, \mathrm{R} 3 \mathrm{C}$, hexangonal axes

Lattice vectors:

○ 1 - 1

$\begin{array}{llll}-1 & \odot & 1\end{array}$

111

origin: $\odot \odot \odot$

\begin{tabular}{|c|c|c|c|c|}
\hline Irrep & Dir & Sub & group & Size \\
\hline GM1+ & (a) & 229 & $I m-3 m$ & 1 \\
\hline GM5+ & $(a, a, a)$ & 166 & $R-3 m$ & \\
\hline GM2 - & (a) & 217 & $\mathrm{I}-43 \mathrm{~m}$ & \\
\hline GM4 - & $(a, a, a)$ & 160 & R3m & \\
\hline $\mathrm{H} 2+$ & (a) & 223 & $P m-3 n$ & \\
\hline $\mathrm{H} 4+$ & $(a, a, a)$ & 167 & $R-3 c$ & \\
\hline H1 - & (a) & 222 & $P n-3 n$ & \\
\hline H5 - & $(a, a, a)$ & 167 & $R-3 c$ & \\
\hline
\end{tabular}

Coupled OP.

\section{ZIZHIZ}

Parent: 194 D6h-4, P6_3/mmc, P6_3/m2/m2/c

Subgroup: $147 \mathrm{C} 3 \mathrm{i}-1, \mathrm{P}-3, \mathrm{P}-3$

Lattice vectors:

100

$\odot \begin{array}{lll}0 & 1 & 0\end{array}$

$\odot \odot 1$

origin: $\odot \odot \odot$

$\begin{array}{lllc}\text { Irrep } & \text { Dir } & \text { Subgroup } & \text { Size } \\ \text { GM1+ } & \text { (a) } & 194 \text { P6_3/mmC } & 1 \\ \text { GM2+ } & \text { (a) } & 176 \text { P6_3/m } & 1 \\ \text { GM3+ } & \text { (a) } & 164 \text { P-3m1 } & 1 \\ \text { GM4+ } & \text { (a) } & 163 \text { P-31c } & 1\end{array}$

Coupled OP.

\section{MTRETC10}

Parent: 225 oh-5, Fm-3m, F4/m-32/m

Subgroup: 152 D3-4, P3_121, P3_121

Lattice vectors:

$0-1 / 2-1 / 2$

$1 / 2 \odot 1 / 2$

$-1-11$

origin: $-1 / 61 / 6-1 / 2$

Irrep k params Dir

GM1+ (a)

GM5+ $\quad(a,-a,-a)$

GM1 - $\quad$ (a)

Subgroup Size

GM5 -

$(\mathrm{a},-\mathrm{a},-\mathrm{a})$

$225 \mathrm{Fm}-3 \mathrm{~m} \quad 1$

$166 \mathrm{R}-3 \mathrm{~m} \quad 1$

LD3 2/3

$(0,0,0,0,0,0, a, 0,0,0,0,0,0,0,-1.732 a, 0)$

209 F432 1

155 R32 1

152 P3_121 3


LD3 is the primary OP.

\section{FUZLUH \& VAFWAA}

Parent: 227 Oh-7, Fd-3m, F4_1/d-32/m, origin choice 2

Subgroup: 141 D4h-19, I4_1/amd, I4_1/a2/m2/d, origin choice 2

Lattice vectors:

$1 / 2-1 / 2 \odot$

$1 / 2 \quad 1 / 20$

$\odot \quad 01$

origin: $1 / 41 / 40$

Irrep Dir Subgroup Size

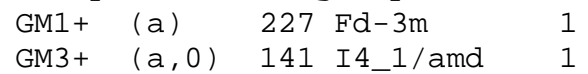

GM3+ is the primary OP.

\section{ZZZKNW01}

Parent: 225 oh-5, Fm-3m, F4/m-32/m

Subgroup: 121 D2d-11, I-42m, I-42m

Lattice vectors:

$-1 / 2 \odot 1 / 2$

$1 / 2 \odot 1 / 2$

010

origin: $\odot \odot \odot$

$\begin{array}{lllc}\text { Irrep Dir } & \text { Subgroup } & \text { Size } \\ \text { GM1+ } & (\mathrm{a}) & 225 \mathrm{Fm}-3 \mathrm{~m} & 1 \\ \text { GM3+ } & (\mathrm{a},-1.732 \mathrm{a}) & 139 \mathrm{I} 4 / \mathrm{mmm} & 1 \\ \text { GM5- } & (0,0, \mathrm{a}) & 121 \mathrm{I}-42 \mathrm{~m} & 1\end{array}$

GM5 - is the primary OP.

\section{KUJSIR}

Parent: 225 Oh-5, Fm-3m, F4/m-32/m

Subgroup: 142 D4h-20, I4_1/acd, I4_1/a2/c2/d, origin choice 2 Lattice vectors:

$1 \odot \odot$

$\begin{array}{llll}0 & 0 & -1\end{array}$

020

origin: $\odot 1 / 41 / 4$

$\begin{array}{lllc}\text { Irrep } & \text { Dir } & \text { Subgroup } & \text { Size } \\ \text { GM1+ } & (\mathrm{a}) & 225 \mathrm{Fm}-3 \mathrm{~m} & 1 \\ \text { GM3+ } & (\mathrm{a},-1.732 \mathrm{a}) & 139 \mathrm{I} 4 / \mathrm{mmm} & 1 \\ \text { X4- } & (\mathrm{a}, 0,0) & 134 \mathrm{P} 4 \_2 / \mathrm{nnm} & 2 \\ \text { W3 } & (0,0, \mathrm{a}, \mathrm{a}, \odot, \odot) & 142 \text { I4_1/acd } & 4\end{array}$

W3 is the primary OP.

\section{YEMRIR}

Parent: $2210 \mathrm{~h}-1, \mathrm{Pm}-3 \mathrm{~m}, \mathrm{P} 4 / \mathrm{m}-32 / \mathrm{m}$

Subgroup: 120 D2d-10, I-4c2, I-4c2

Lattice vectors:

$1-10$

110

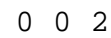

origin: $-1 / 2-1 / 2-1 / 2$

$\begin{array}{lllc}\text { Irrep Dir } & \text { Subgroup } & \text { Size } \\ \text { GM1+ } & (\mathrm{a}) & 221 \mathrm{Pm}-3 \mathrm{~m} & 1 \\ \text { GM3+ } & (\mathrm{a}, 0) & 123 \mathrm{P} 4 / \mathrm{mmm} & 1 \\ \text { GM2 - } & (\mathrm{a}) & 215 \mathrm{P}-43 \mathrm{~m} & 1 \\ \text { GM3- } & (\mathrm{a}, 0) & 111 \mathrm{P}-42 \mathrm{~m} & 1 \\ \text { R4+ } & (\mathrm{a}, 0,0) & 140 \mathrm{I} 4 / \mathrm{mcm} & 2 \\ \text { R5- } & (\mathrm{a}, \odot, \odot) & 140 \mathrm{I} 4 / \mathrm{mcm} & 2\end{array}$

Coupled OP. 
ADAMAN08 \& GERHOA

Parent: 225 Oh-5, Fm-3m, F4/m-32/m

Subgroup: 114 D2d-4, P-42_1C, P-42_1C

Lattice vectors:

$-1 / 2 \odot 1 / 2$

$1 / 2 \odot 1 / 2$

010

origin: $\odot \odot \odot$

\begin{tabular}{lllc} 
Irrep Dir & \multicolumn{2}{c}{ Subgroup } & Size \\
GM1+ & $(\mathrm{a})$ & $225 \mathrm{Fm}-3 \mathrm{~m}$ & 1 \\
GM3+ & $(\mathrm{a},-1.732 \mathrm{a})$ & $139 \mathrm{I} 4 / \mathrm{mmm}$ & 1 \\
GM5- & $(0,0, \mathrm{a})$ & $121 \mathrm{I}-42 \mathrm{~m}$ & 1 \\
X3+ & $(\mathrm{a}, 0,0)$ & $128 \mathrm{P} 4 / \mathrm{mnc}$ & 2 \\
X2- & $(\mathrm{a}, 0,0)$ & $137 \mathrm{P}$ - $2 / \mathrm{nmc}$ & 2
\end{tabular}

Coupled OP.

\section{KANGUBO1}

Parent: 141 D4h-19, I4_1/amd, I4_1/a2/m2/d, origin choice 2

Subgroup: 88 C4h-6, I4_1/a, I4_1/a, origin choice 2

Lattice vectors:

100

$\begin{array}{llll}0 & 1 & 0\end{array}$

$\odot \begin{array}{ccc}0 & 0 & 1\end{array}$

origin: $\odot 1 / 2 \odot$

Irrep Dir Subgroup Size

GM1+ (a) 141 I4_1/amd 1

GM3+ (a) 88 I4_1/a 1

GM3+ is the primary OP.

(methane III)

Parent: 225 oh-5, Fm-3m, F4/m-32/m

Subgroup: 64 D2h-18, Cmca, C2/m2/c2_1/a

Lattice vectors:

200

$\odot \begin{array}{lll}0 & 1 & 1\end{array}$

$\begin{array}{llll}0 & -1 & 1\end{array}$

origin: $1 / 2 \odot \odot$

Irrep k params Dir

GM1+ (a)

GM3+ $\quad(a, 1.732 a)$

GM5+ $(0, \mathrm{a}, \odot)$

SM2 $1 / 4 \quad(\odot, \odot, \odot, \odot, \odot, \odot, \odot, \odot, \odot, \odot, \mathrm{a}, \odot)$

L1- $\quad(a, 0, a, 0)$

L3- $\quad(a, 0.268 a, 0, \odot, a, 0.268 a, \odot, \odot)$

$\mathrm{X} 1+\quad(0,0, \mathrm{a})$

$\mathrm{X} 4+\quad(0,0, \mathrm{a})$

W2 $\quad(a, 0,0,0,0,0)$

W3 $\quad(\odot, a, 0,0,0, \odot)$

$\begin{array}{lc}\text { Subgroup } & \text { Size } \\ 225 \text { Fm-3m } & 1 \\ 139 \text { I4/mmm } & 1 \\ 71 \text { Immm } & 1 \\ 51 \mathrm{Pmma} & 4 \\ 67 \mathrm{Cmma} & 4 \\ 67 \mathrm{Cmma} & 4 \\ 123 \mathrm{P} 4 / \mathrm{mmm} & 2 \\ 131 \mathrm{P} 422 / \mathrm{mmc} & 2 \\ 139 \mathrm{I} 4 / \mathrm{mmm} & 4 \\ 140 \mathrm{I} 4 / \mathrm{mcm} & 4\end{array}$

Coupled OP.

\section{YIMWEW}

Parent: 229 oh-9, Im-3m, I4/m-32/m

Subgroup: $60 \mathrm{D} 2 \mathrm{~h}-14, \mathrm{Pbcn}, \mathrm{P} 2 \_1 / \mathrm{b} 2 / \mathrm{c} 2 \_1 / \mathrm{n}$

Lattice vectors:

○ 30

$\begin{array}{llll}-1 & 0 & 1\end{array}$

$1 \odot 1$

origin: $-1 / 2 \odot \odot$

$\begin{array}{ll}\text { Irrep k params } & \text { Dir } \\ \text { GM1+ } & (a) \\ \text { GM3+ } & (a,-1.732 a) \\ \text { GM5+ } & (\odot, \odot, a)\end{array}$

\begin{tabular}{|c|c|c|}
\hline Sul & group & Size \\
\hline 22 & Im $-3 m$ & 1 \\
\hline 13 & $\mathrm{I} 4 / \mathrm{mmm}$ & 1 \\
\hline 6 & Fmmm & 1 \\
\hline
\end{tabular}




$\begin{array}{lllrl}\text { DT5 } & 5 / 6 & (\mathrm{a},-\mathrm{a}, \mathrm{a}, \mathrm{a}, \odot, \odot, \odot, \odot, \odot, \odot, \odot, \odot) & 64 \mathrm{Cmca} & 6 \\ \text { DT1 } & 1 / 3 & (\mathrm{a}, \odot, \odot, \odot, \odot, \odot) & 139 \mathrm{I} 4 / \mathrm{mmm} & 3 \\ \text { DT3 } & 1 / 3 & (\odot, \mathrm{a}, \odot, \odot, \odot, \odot) & 69 \mathrm{Fmmm} & 3 \\ \text { H4+ } & & (\mathrm{a},-\mathrm{a}, \odot) & 64 \mathrm{Cmca} & 2 \\ \text { H5+ } & (\mathrm{a}, \mathrm{a}, \odot) & 64 \mathrm{Cmca} & 2 \\ \text { N1- } & (\odot, \odot, \mathrm{a}, \odot, \odot, \odot) & 68 \mathrm{Ccca} & 2 \\ \text { N4- } & (\odot, \odot, \mathrm{a}, \odot, \odot, \odot) & 63 \mathrm{Cmcm} & 2 \\ \text { D2 } & 1 / 6 & (\odot, \odot, \mathrm{a}, \odot, \odot, \odot, \odot, \odot, \odot, \odot, \odot, \odot) & 68 \mathrm{Ccca} & 6 \\ \text { D3 } & 1 / 6 & (\odot, \odot, \odot, \odot, \odot, \odot, \odot, \odot, \odot, \mathrm{a}, \odot, \odot) & 63 \mathrm{Cmcm} & 6\end{array}$

Coupled OP.

\section{RASDOE \& TFMETHO2}

Parent: 70 D2h-24, Fddd, F2/d2/d2/d, origin choice 2

Subgroup: $15 \mathrm{C} 2 \mathrm{~h}-6, \mathrm{C} 2 / \mathrm{c}, \mathrm{C} 12 / \mathrm{c} 1$, unique axis $\mathrm{b}$, cell choice 1 Lattice vectors:

$0-10$

$\begin{array}{llll}-1 & 0 & 0\end{array}$

$01 / 2-1 / 2$

origin: $1 / 4 \odot 1 / 4$

Irrep Dir Subgroup Size

GM1+ (a) 70 Fddd 1

GM3+ (a) $\quad 15 \mathrm{C} 2 / \mathrm{C} \quad 1$

GM3+ is the primary OP.

\section{REKYUB}

Parent: 225 oh-5, Fm-3m, F4/m-32/m

Subgroup: $15 \mathrm{C} 2 \mathrm{~h}-6, \mathrm{C} 2 / \mathrm{c}, \mathrm{C} 12 / \mathrm{c} 1$, unique axis b, cell choice 1 Lattice vectors:

$-1 / 2 \quad 1-1 / 2$

$\begin{array}{lll}-1 / 2 & 0 & 1 / 2\end{array}$

101

origin: $-1 / 4 \quad 0 \quad-1 / 4$

$\begin{array}{llrc}\text { Irrep Dir } & \text { Subgroup } & \text { Size } \\ \text { GM1+ } & (\mathrm{a}) & 225 \mathrm{Fm}-3 \mathrm{~m} & 1 \\ \text { GM3+ } & (\mathrm{a},-1.732 \mathrm{a}) & 139 \mathrm{I} 4 / \mathrm{mmm} & 1 \\ \text { GM4+ } & (\mathrm{a}, 0,-\mathrm{a}) & 12 \mathrm{C} 2 / \mathrm{m} & 1 \\ \text { GM5+ } & (\mathrm{a}, \mathrm{a}, \mathrm{b}) & 12 \mathrm{C} 2 / \mathrm{m} & 1 \\ \text { L1- } & (\mathrm{a}, \odot, \odot, \odot) & 167 \mathrm{R}-3 \mathrm{c} & 2 \\ \text { L3- }(\mathrm{a}, 3.732 \mathrm{a}, \odot, \odot, \odot, \odot, \odot, \odot) & 15 \mathrm{C} 2 / \mathrm{c} & 2\end{array}$

L3 - is the primary OP.

\section{MECKOU}

Parent: 225 oh $-5, F m-3 m, F 4 / m-32 / m$

Subgroup: $12 \mathrm{c} 2 \mathrm{~h}-3, \mathrm{C} 2 / \mathrm{m}, \mathrm{C} 12 / \mathrm{m} 1$, unique axis b, cell choice 1 Lattice vectors:

$1 / 2-1 / 2-1$

$1 / 21 / 20$

$1 / 2-1 / 21$

origin: $-1 / 41 / 40$

$\begin{array}{llrc}\text { Irrep Dir } & \text { Subgroup } & \text { Size } \\ \text { GM1+ } & (\mathrm{a}) & 225 \mathrm{Fm}-3 \mathrm{~m} & 1 \\ \text { GM3+ } & (\mathrm{a}, \odot) & 139 \mathrm{I} 4 / \mathrm{mmm} & 1 \\ \text { GM4+ } & (\mathrm{a}, \mathrm{a}, \odot) & 12 \mathrm{C} 2 / \mathrm{m} & 1 \\ \text { GM5+ } & (\mathrm{a}, \mathrm{b},-\mathrm{b}) & 12 \mathrm{C} 2 / \mathrm{m} & 1 \\ \text { L2 }- & (\odot, \odot, \odot, \mathrm{a}) & 166 \mathrm{R}-3 \mathrm{~m} & 2 \\ \text { L3 }-(\odot, \odot, \odot, \odot, \odot, \odot, \mathrm{a}, \mathrm{a}) & 12 \mathrm{C} 2 / \mathrm{m} & 2\end{array}$

L3- is the primary OP.

\section{MECKUA}

Parent: 225 oh-5, Fm-3m, F4/m-32/m

Subgroup: $14 \mathrm{C} 2 \mathrm{~h}-5, \mathrm{P} 2 \_1 / \mathrm{c}, \mathrm{P} 12 \_1 / \mathrm{c} 1$, unique axis b, cell choice 1 Lattice vectors:

$1 / 2 \quad 1-1 / 2$ 


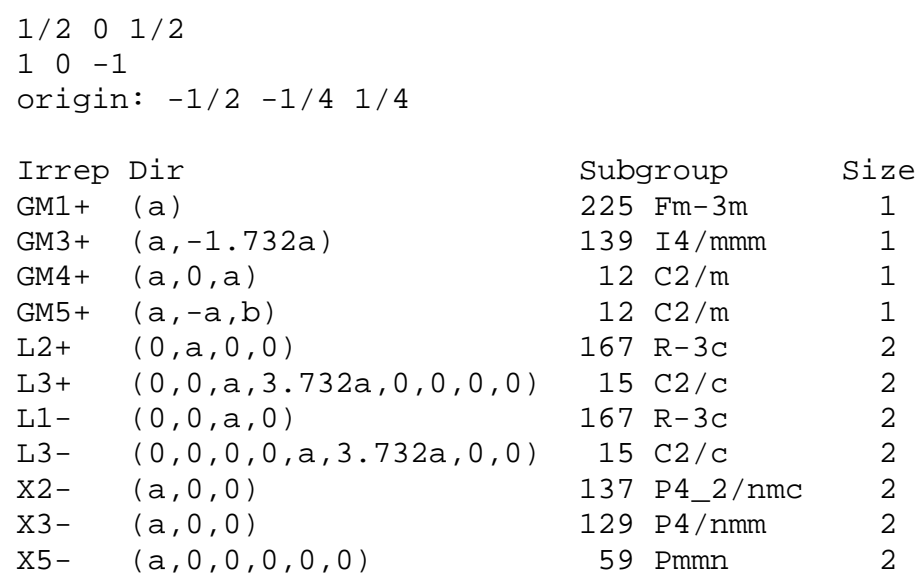

Coupled OP.

\section{TOHSUE}

Parent: 225 Oh-5, Fm-3m, F4/m-32/m

Subgroup: $14 \mathrm{C} 2 \mathrm{~h}-5, \mathrm{P} 2 \_1 / \mathrm{c}, \mathrm{P} 12 \_1 / \mathrm{c} 1$, unique axis b, cell choice 1 Lattice vectors:

$-1 / 2 \odot 1 / 2$

$1 / 2 \odot 1 / 2$

020

origin: $\odot-1 / 4-1 / 4$

\begin{tabular}{|c|c|c|c|c|c|}
\hline Irrep & k params & Dir & Sub & group & Size \\
\hline GM1+ & & (a) & 225 & $F m-3 m$ & 1 \\
\hline GM3+ & & $(a,-1.732 a)$ & 139 & $\mathrm{I} 4 / \mathrm{mmm}$ & 1 \\
\hline GM4+ & & $(a, 0, a)$ & 12 & $\mathrm{C} 2 / \mathrm{m}$ & 1 \\
\hline GM5+ & & $(a,-a, b)$ & 12 & $\mathrm{C} 2 / \mathrm{m}$ & 1 \\
\hline DT2 & $3 / 4$ & $(a,-a, \odot, \odot, \odot, \odot)$ & 138 & P4_2/ncm & 4 \\
\hline DT4 & $3 / 4$ & $(a, a, 0,0,0,0)$ & 130 & $\mathrm{P} 4 / \mathrm{ncc}$ & 4 \\
\hline DT5 & $3 / 4$ & $(\odot, a,-a, 0,0, \odot, \odot, \odot, \odot, \odot, \odot, \odot)$ & 62 & Pnma & 4 \\
\hline$X 2-$ & & $(a, 0,0)$ & 137 & P4_2/nmc & 2 \\
\hline X3- & & $(a, 0,0)$ & 129 & $\mathrm{P} 4 / \mathrm{nmm}$ & 2 \\
\hline$x 5-$ & & $(a, 0, \Theta, \odot, \Theta, \Theta)$ & 59 & Pmmn & 2 \\
\hline
\end{tabular}

Coupled OP.

\section{CARBTC07 \& CTBROM}

Parent: 225 Oh-5, Fm-3m, F4/m-32/m

Subgroup: $15 \mathrm{C} 2 \mathrm{~h}-6, \mathrm{C} 2 / \mathrm{c}, \mathrm{C} 12 / \mathrm{c} 1$, unique axis b, cell choice 1 Lattice vectors:

$\begin{array}{lll}-2 & -1 & -1\end{array}$

$\begin{array}{llll}0 & 1 & -1\end{array}$

$2-1-1$

origin: $-1 / 21 / 21 / 2$

Irrep k params Dir

\begin{tabular}{|c|c|c|}
\hline & (a) \\
\hline GM3+ & & $(a, 1.732 a)$ \\
\hline GM4+ & & $(0, a,-a)$ \\
\hline GM5+ & & $(a, b, a)$ \\
\hline LD2 & $3 / 4$ & $(\odot, a, 0,0,0,-a, 0,0)$ \\
\hline LD3 & $3 / 4$ & $(\odot, \odot, a, \odot .268 a, \odot, \odot, \odot, \odot, \odot, \odot, \odot .268 a, a, \odot, \odot, \odot, \odot)$ \\
\hline L1- & & $(0, a, 0, a)$ \\
\hline L2 - & & $(a, b, c,-b)$ \\
\hline L3 - & & $(a,-3.732 a, b, c, d,-3.732 d, 0.866 b+0.500 c, 0.500 b-0.866 c)$ \\
\hline $\mathrm{X} 1+$ & & $(a,-a, b)$ \\
\hline $\mathrm{X} 2+$ & & $(a, a, 0)$ \\
\hline $\mathrm{x} 3+$ & & $(a, a, 0)$ \\
\hline $\mathrm{X} 4+$ & & $(a,-a, b)$ \\
\hline$x 5+$ & & $(a, b,-b, a, 0, c)$ \\
\hline C1 & $1 / 2,1 / 4$ & 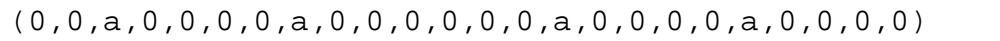 \\
\hline $\mathrm{C} 2$ & $1 / 2,1 / 4$ & $(\odot, 0, a, 0,0,0,0,-a, b, 0,0,0,0,0,-a, 0,0,0,0, a,-b, 0,0,0)$ \\
\hline
\end{tabular}

\begin{tabular}{|c|c|c|}
\hline sub & aroup & $\mathrm{Siz}$ \\
\hline 225 & $F m-3 m$ & 1 \\
\hline 139 & $\mathrm{I} 4 / \mathrm{mmm}$ & 1 \\
\hline 12 & $\mathrm{C} 2 / \mathrm{m}$ & \\
\hline 12 & $\mathrm{C} 2 / \mathrm{m}$ & \\
\hline 167 & $R-3 c$ & \\
\hline 15 & $\mathrm{C} 2 / \mathrm{C}$ & \\
\hline 67 & Cmma & \\
\hline 12 & $\mathrm{C} 2 / \mathrm{m}$ & 8 \\
\hline 12 & $\mathrm{C} 2 / \mathrm{m}$ & 8 \\
\hline 123 & $\mathrm{P} 4 / \mathrm{mmm}$ & \\
\hline 123 & $\mathrm{P} 4 / \mathrm{mmm}$ & \\
\hline 134 & P4_2/nnm & 4 \\
\hline 134 & P4_2/nnm & \\
\hline 12 & $\mathrm{C} 2 / \mathrm{m}$ & \\
\hline 12 & $\mathrm{C} 2 / \mathrm{m}$ & 列 \\
\hline 15 & $\mathrm{C} 2 / \mathrm{C}$ & 16 \\
\hline
\end{tabular}

C2 is the primary oP. 
MEZDIE01 \& MEZDOKO1

Parent: 229 Oh-9, Im-3m, I4/m-32/m

Subgroup: $2 \mathrm{Ci}-1, \mathrm{P}-1, \mathrm{P}-1$

Lattice vectors:

$\begin{array}{lll}1 / 2 & 1 / 2 & 1 / 2\end{array}$

$100-1$

$-1 / 21 / 2-1 / 2$

origin: $-1 / 4-1 / 41 / 4$

$\begin{array}{llrc}\text { Irrep } & \text { Dir } & \text { Subgroup } & \text { Size } \\ \text { GM1+ } & (\mathrm{a}) & 229 \mathrm{Im}-3 \mathrm{~m} & 1 \\ \text { GM2+ } & (\mathrm{a}) & 204 \mathrm{Im}-3 & 1 \\ \text { GM3+ } & (\mathrm{a}, \mathrm{b}) & 71 \mathrm{Immm} & 1 \\ \text { GM4+ } & (\mathrm{a}, \mathrm{b}, \mathrm{c}) & 2 \mathrm{P}-1 & 1 \\ \text { GM5+ } & (\mathrm{a}, \mathrm{b}, \mathrm{c}) & 2 \mathrm{P}-1 & 1 \\ \text { N1- } & (\odot, \odot, \mathrm{a}, \odot, \odot, \odot) & 68 \mathrm{Ccca} & 2 \\ \text { N2- } & (\odot, \odot, \odot, \mathrm{a}, \odot, \odot) & 63 \mathrm{Cmcm} & 2 \\ \text { N3- } & (\odot, \odot, \mathrm{a}, \odot, \odot, \odot) & 67 \mathrm{Cmma} & 2 \\ \text { N4- } & (\odot, \odot, \odot, a, \odot, \odot) & 63 \mathrm{Cmcm} & 2\end{array}$

Coupled OP.

\section{OHABEE}

Parent: 229 oh-9, Im-3m, I4/m-32/m

Subgroup: $2 \mathrm{Ci}-1, \mathrm{P}-1, \mathrm{P}-1$

Lattice vectors:

$1 / 21 / 21 / 2$

$1-10$

$\begin{array}{lll}1 & 1 & -2\end{array}$

origin: $-1-1 / 21$

Irrep k params Dir

GM1+ (a)

GM2+ (a)

GM3+ $\quad(a, b)$

GM4+ $\quad(a, b, c)$

GM5+ $(a, b, c)$

LD1 $\quad 1 / 3 \quad(a, 0,0,0,-1.732 a, 0,0, \odot)$

LD2 $\quad 1 / 3 \quad(a, \odot, \odot, \odot, \odot .577 a, \odot, \odot, \odot)$

LD3 $1 / 3 \quad(a, b, \odot, \odot, \odot, \odot, \odot, \odot,-\odot .577 a-1.155 b,-1.155 a-\odot .577 b, \odot, \odot, \odot, \odot, \odot, \odot)$

N1 - $\quad(0,0,0,0, a, 0)$

N2- $\quad(0,0,0,0,0, \mathrm{a})$

N3 - $\quad(\odot, \odot, \odot, \odot, a, \odot)$

N4 - $\quad(0,0,0,0,0, a)$

C1 $1 / 6,2 / 3 \quad(a, 0,0,0,0,0,0,0,0,0,0,0,-\odot .577 a, 0,0,0,0,0,0,0,0,0,0,0)$

C2 $1 / 6,2 / 3$

$(\mathrm{a}, \odot, \odot, \odot, \odot, \odot, \odot, \odot, \odot, \odot, \odot, \odot, 1.732 \mathrm{a}, \odot, \odot, \odot, \odot, \odot, \odot, \odot, \odot, \odot, \odot, \odot)$

\begin{tabular}{|c|c|}
\hline Sub & yroup \\
\hline 229 & Im-3m \\
\hline 204 & Im-3 \\
\hline 71 & Immm \\
\hline 2 & $P-1$ \\
\hline 2 & $P-1$ \\
\hline 164 & $P-3 m 1$ \\
\hline 147 & $P-3$ \\
\hline 2 & $P-1$ \\
\hline 68 & Ccca \\
\hline 63 & $\mathrm{Cmcm}$ \\
\hline 67 & Cmma \\
\hline 63 & $\mathrm{Cmcm}$ \\
\hline 11 & P2_1/m \\
\hline 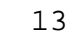 & $\mathrm{P} 2 / \mathrm{C}$ \\
\hline
\end{tabular}

Coupled OP. 


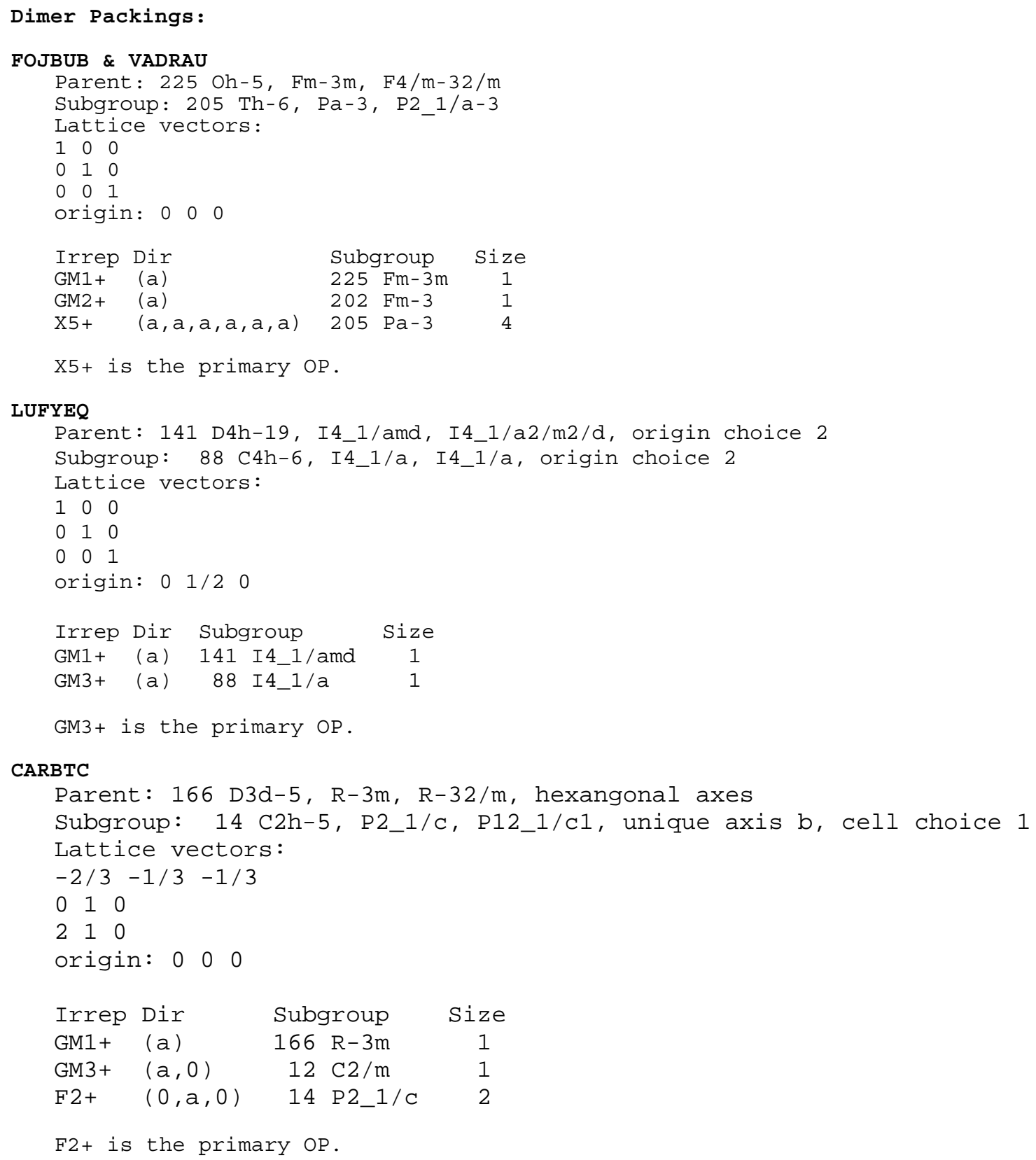


Mixed

KOXKOX, RUQMEV, \& SENLAY

Parent: 223 oh-3, Pm-3n, P4_2/m-32/n

Subgroup: $218 \mathrm{Td}-4, \mathrm{P}-43 \mathrm{n}, \mathrm{P}-43 \mathrm{n}$

Lattice vectors:

100

010

$\odot \quad 01$

origin: $\odot \odot \odot$

Irrep Dir Subgroup Size

GM1+ (a) 223 Pm-3n 1

GM2 - (a) 218 P-43n 1

GM2 - is the primary OP. 
Table 9. Identifiers, chemical formulas, and assigned frameworks of the tetrahedral data set. The adamantane and cubane molecular frameworks are substituted derivatives of these molecules, while $\mathrm{MX}_{4}$ molecules have a central atom (denoted $\mathrm{M}$ ) with four identical X groups.

\begin{tabular}{|c|c|c|}
\hline Identifier & formula & framework \\
\hline ADAMAN08 & $\mathrm{C} 10 \mathrm{H} 16$ & adamantane \\
\hline BASXOI & C4 H12 Se6 Sn 4 & adamantane \\
\hline BOGMEP & $\mathrm{C} 24 \mathrm{H} 48 \mathrm{Cl} 6 \mathrm{Cu} 4 \mathrm{~N} 16 \mathrm{O} 1$ & other \\
\hline CAMPOV & C 16 H36 N4 Sn4 & cubane \\
\hline CANFIG & $\mathrm{C} 4 \mathrm{H} 24 \mathrm{~B} 4 \mathrm{U} 1$ & $\mathrm{MX}_{4}$ \\
\hline CANFOM & C4 H24 B4 Th1 & $\mathrm{MX}_{4}$ \\
\hline CARBTC & $\mathrm{C} 1 \mathrm{Cl} 4$ & $\mathrm{MX}_{4}^{+}$ \\
\hline CARBTC07 & $\mathrm{C} 1 \mathrm{Cl} 4$ & $\mathrm{MX}_{4}$ \\
\hline CTBROM & $\mathrm{C} 1 \mathrm{Br} 4$ & $\mathrm{MX}_{4}$ \\
\hline CUCZUV & C20 H36 & tetrahedrane \\
\hline DEQPAQ & C 36 H100 B4 N12 Na4 & other \\
\hline DILWIE01 & $\mathrm{C} 16 \mathrm{H} 48 \mathrm{Pt} 4 \mathrm{~S} 4$ & cubane \\
\hline DOCNIS & $\mathrm{C} 8 \mathrm{H} 12 \mathrm{~S} 6$ & adamantane \\
\hline FOHCUA & $\mathrm{C} 12 \mathrm{Ni}_{4} \mathrm{O} 18 \mathrm{P} 4$ & adamantane \\
\hline FOJBUB02 & $\mathrm{C} 4 \mathrm{Ni} 1 \mathrm{O} 4$ & $\mathrm{MX}_{4}$ \\
\hline FUZLUH & $\mathrm{C} 12 \mathrm{Co} 4 \mathrm{O} 12 \mathrm{Sb} 4$ & cubane \\
\hline FUZTEZ & H16 B4 Np1 & $\mathrm{MX}_{4}$ \\
\hline FUZVOL & H16 B4 Hf1 & $\mathrm{MX}_{4}$ \\
\hline GERHOA & $\mathrm{C} 4 \mathrm{H} 12 \mathrm{Cl}_{12} \mathrm{~N} 4 \mathrm{Sb} 4$ & cubane \\
\hline GUTCED & $\mathrm{C} 26 \mathrm{H} 32$ & other \\
\hline HMGETP & $\mathrm{C} 12 \mathrm{H} 36 \mathrm{Ge} 6 \mathrm{P} 4$ & adamantane \\
\hline HMSIPA & $\mathrm{C} 12 \mathrm{H} 36 \mathrm{P} 4 \mathrm{Si} 6$ & adamantane \\
\hline HXMTAM07 & C6 $\mathrm{H} 12 \mathrm{~N} 4$ & adamantane \\
\hline JEYSEL & C18 H36 Ni4 O6 P4 & tetrahedrane \\
\hline JUFWUC & $\mathrm{C} 12 \mathrm{H} 40 \mathrm{Cs} 4 \mathrm{~N} 4 \mathrm{Si} 4$ & cubane \\
\hline KANGUB01 & $\mathrm{C} 10 \mathrm{H} 12 \mathrm{I} 4$ & adamantane \\
\hline KELREY & $\mathrm{C} 12 \mathrm{H} 36 \mathrm{Cl} 4 \mathrm{Ti} 4$ & cubane \\
\hline KOXKOX & C16 H36 Ga4 Se4 & cubane \\
\hline KUJSIR & $\mathrm{C} 20 \mathrm{H} 48 \mathrm{O} 4 \mathrm{Zn} 4$ & cubane \\
\hline LUFYEQ & $\mathrm{C} 12 \mathrm{H} 12 \mathrm{Si} 1$ & $\mathrm{MX}_{4}$ \\
\hline MECKIO & C16 H36 Cl4 In 4 N4 & cubane \\
\hline MECKOU & C16 H36 Br4 In4 N4 & cubane \\
\hline MECKUA & C16 H 36 I 4 In 4 N 4 & cubane \\
\hline MESIAD & C12 H36 As4 Si6 & adamantane \\
\hline MEZDIE01 & C12 H36 Si1 Sn4 & $\mathrm{MX}_{4}$ \\
\hline MEZDOK01 & C12 H36 Ge1 Sn 4 & $\mathrm{MX}_{4}$ \\
\hline MPTHOTO1 & $\mathrm{C} 12 \mathrm{H} 40 \mathrm{O} 4 \mathrm{Pt} 4$ & cubane \\
\hline MSISUL10 & $\mathrm{C} 4 \mathrm{H} 12 \mathrm{~S} 6 \mathrm{Si} 4$ & adamantane \\
\hline MTRETC10 & C 16 H12 O12 Re 4 S 4 & cubane \\
\hline MXSNOX & $\mathrm{C} 4 \mathrm{H} 12$ O8 Sn 6 & other \\
\hline MZNMOX 10 & $\mathrm{C} 8 \mathrm{H} 24 \mathrm{O} 4 \mathrm{Zn} 4$ & cubane \\
\hline NIWMIP & $\mathrm{C} 12 \mathrm{H} 36 \mathrm{Al} 4 \mathrm{~N} 4 \mathrm{~S} 6$ & adamantane \\
\hline OHABEE & C16 H36 Si4 & tetrahedrane \\
\hline POSLOY 10 & $\mathrm{C} 12 \mathrm{Cl} 4 \mathrm{O} 12 \mathrm{Tc} 4$ & cubane \\
\hline QUGBOJ & C16 O16 Rh6 & other \\
\hline RASDOE & C16 H48 Ga4 N4 Si4 & cubane \\
\hline REKYUB & C16 H36 Ga4 S4 & cubane \\
\hline RIMMOP & C16 H40 Al4 N4 & cubane \\
\hline RIMNAC & $\mathrm{C} 20 \mathrm{H} 48$ Al4 N4 & cubane \\
\hline RUQMEV & $\mathrm{C} 12 \mathrm{H} 36 \mathrm{Cu} 4 \mathrm{I} 4 \mathrm{~N} 4$ & cubane \\
\hline SENLAY & C16 H36 P4 Si4 & cubane \\
\hline TCYMET & C5 N4 & $\mathrm{MX}_{4}$ \\
\hline TFMETH02 & $\mathrm{C} 1 \mathrm{~F} 4$ & $\mathrm{MX}_{4}$ \\
\hline TMEPTC & C12 H36 Cl4 Pt4 & cubane \\
\hline TMGEHS10 & $\mathrm{C} 4 \mathrm{H} 12 \mathrm{Ge} 4 \mathrm{~S} 6$ & adamantane \\
\hline TMSIAD & $\mathrm{C} 10 \mathrm{H} 24 \mathrm{Si} 4$ & adamantane \\
\hline TMSNHS10 & C4 H12 S6 Sn 4 & adamantane \\
\hline TOHSUE & C16 F12 O12 P4 Ru 4 & cubane \\
\hline VADRAU & $\mathrm{C} 4 \mathrm{H} 12 \mathrm{~Pb} 1$ & $\mathrm{MX}_{4}$ \\
\hline VAFWAA & $\mathrm{C} 12 \mathrm{Bi} 4 \mathrm{Co} 4 \mathrm{O} 12$ & cubane \\
\hline VAVYAS & $\mathrm{C} 20 \mathrm{H} 36 \mathrm{P} 4$ & cubane \\
\hline XAGXAE & P4 S10 & adamantane \\
\hline XUWROW & $\mathrm{C} 20 \mathrm{H} 48 \mathrm{Mg} 4 \mathrm{O} 4$ & cubane \\
\hline YEMRIR & $\mathrm{O} 6 \mathrm{P} 4 \mathrm{~S} 4$ & adamantane \\
\hline YEYQAU & $\mathrm{C} 12 \mathrm{O} 12 \mathrm{Ru} 4 \mathrm{Se} 4$ & cubane \\
\hline YIMWEW & C10 H16 O4 & adamantane \\
\hline ZEYHIU & $\mathrm{C} 20 \mathrm{H} 48 \mathrm{Cd} 4 \mathrm{O} 4$ & cubane \\
\hline ZIZHIZ & $\mathrm{C} 12 \mathrm{H} 4 \mathrm{Mn} 4 \mathrm{O} 16$ & cubane \\
\hline ZNOXAC01 & C12 H18 O13 Zn4 & other \\
\hline ZZZKDW01 & $\mathrm{C} 1 \mathrm{I} 4$ & $\mathrm{MX}_{4}$ \\
\hline
\end{tabular}

